\title{
Improving VR Selection Using Progressive Refinement with Multi-Touch Marking Menus
}

\author{
by
}

Elaheh Samimi

A thesis submitted to the Faculty of Graduate and Postdoctoral Affairs in partial fulfillment of the requirements for the degree of

Master of Information Technology

in

Digital Media

Carleton University

Ottawa, Ontario

(C) 2021, Elaheh Samimi 


\begin{abstract}
Selection is the process of acquiring targets for subsequent operations (e.g., moving or rotating them) in Virtual Reality (VR) systems. Many selection techniques have been introduced to improve selection performance; nonetheless, 3D selection is still cumbersome. We developed a technique to improve selection time and accuracy concurrently by combining progressive refinement and CountMarks. Our technique, Multi-Touch Progressive Refinement (MTPR), enhanced conventional progressive refinement's quad menu object selection by using CountMarks, a multi-finger touchbased technique previously developed to facilitate marking menu selection on smartphones.
\end{abstract}

In a first user study, we compared our technique with progressive refinement and multi-touch techniques in terms of search time, selection time, and accuracy. The results showed that the multi-touch was fastest and progressive refinement was most accurate. However, we also found that participants were slightly confused by our MTPR technique. Therefore, we enhanced our technique by ordering objects inside menu and labelling them. We ran a second study comparing our Improved Multi-Touch Progressive Refinement Selection Strategy (IMPReSS) with the previous two techniques. The result demonstrated that IMPReSS was both the fastest and most accurate of all the techniques evaluated. 


\section{Acknowledgments}

This is the best opportunity for me to thank my wonderful supervisor and mentor, Dr. Robert J. Teather, for his brilliant and spectacular guidance and support. This thesis would not be possible without his wise pieces of advice and endless assistance. $\mathrm{He}$ supported me not only in educational and technical aspects but also in improving my communicational skills, such as academic writing. Also, I would love to thank Dr. Audrey Girouard for her kind support, especially during the COVID-19 pandemic. She trusted me and joined me in the Collaborative Learning of Usability Experiences (CLUE) group, and continuing this education would not be possible without their financial support.

My special thanks to my friend, Yasin Farmani, who helped me get my admission and always supported me during my education time. His kindness will always be inspiring for the rest of my life. I also need to thank all of my amazing labmates who helped me grow academically and personally during these two years. We spent wonderful times together full of joy and laughter in the lab, and all those moments will be memorable for me forever. My thanks also to Daniela Flores Javier and all other researchers I used or inspired by their research.

Last but not least, my deepest thanks to my parents and lovely husband for their support and encouragement. Without their support and sacrifice, it would have impossible for me to finish my higher education. To my family and especially to my husband, I will never forget your kindness and thanks for believing in me. 


\section{Table of Contents}

Abstract.................................................................................................................................. ii

Acknowledgments ................................................................................................................ii

Table of Contents ....................................................................................................................... iv

List of Figures..................................................................................................................................... vi

List of Appendices........................................................................................................................ $\mathrm{x}$

Chapter 1: Introduction........................................................................................

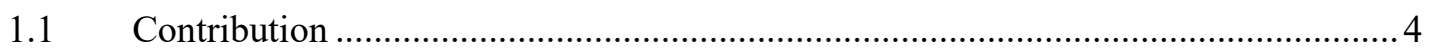

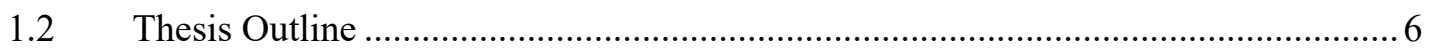

Chapter 2: Literature Review .........................................................................................

$2.1 \quad$ Immediate Selection ....................................................................................

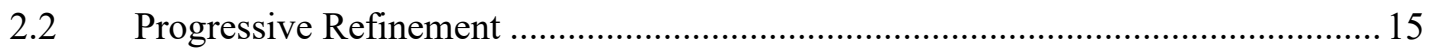

2.3 Marking Menus and Count Menus........................................................................22

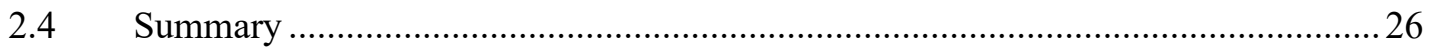

Chapter 3: Design and Implementation of Multi-Touch Progressive Refinement ...................22

3.1 Design and Implementation of MTPR …………………………………………....2

3.2 Menu Distribution Variations ………………………………………………….... 32

3.3 User Study 1: Performance Evaluation of Multi-Touch Progressive Refinement ...33

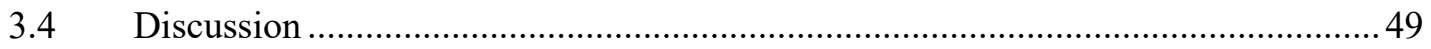

3.5 User Study Limitations ………………………………………………………5

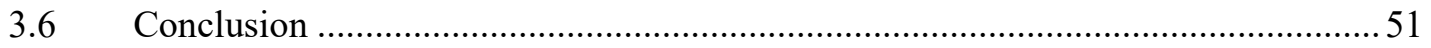

Chapter 4: Design and Implementation of IMPReSS Technique - Improved Multi-Touch

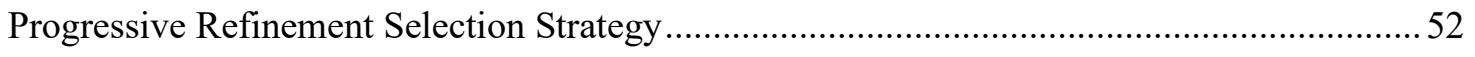

4.1 Distributing Selected Objects Inside the Quad Menu ..............................................52 


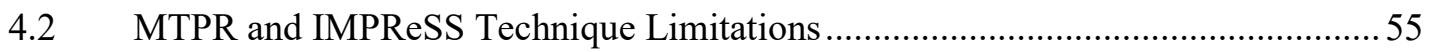

4.3 User Study 2: Evaluating the IMPReSS Technique ...........................................56

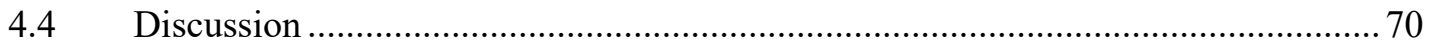

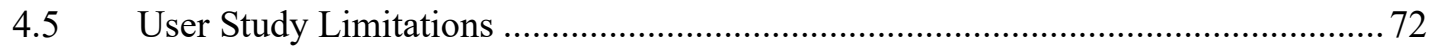

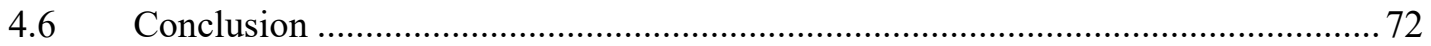

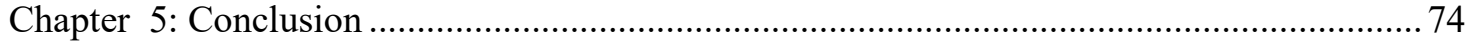

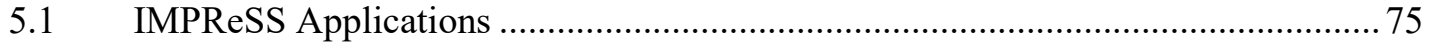

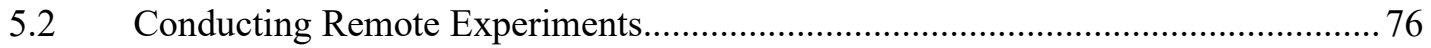

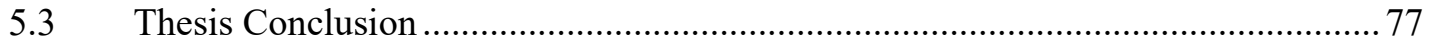

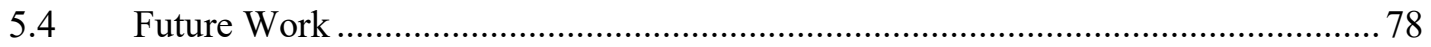

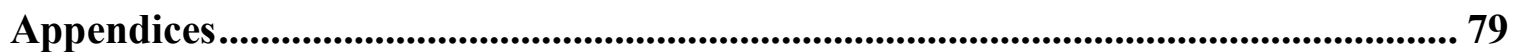

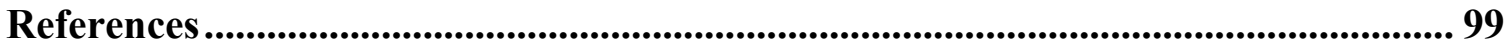




\section{List of Figures}

Figure 1: Immediate selection categories based on task decomposition [37]................... 8

Figure 2: Left) Progressive refinement first step selection; selecting target area. Right) Progressive refinement second step, distributing selected objects inside a quad menu [30].

Figure 3: With the Expand technique, selectable objects remain in their original context while clones are displayed in a virtual grid for making a final selection [37]. 18

Figure 4: Three refinement steps of the discrete zoom technique [4], left) the first zoom level, middle) the second zoom level, right) the third zoom level. 19

Figure 5: PRECIOUS technique: (a) selection cone intersecting various objects, (b) refinement phase, moving the user closer to the objects, (c) single object selection, (d) returning to the original position with the object selected [40]. 20

Figure 6: Three snapshots of different zoom levels achieved with the continuous zoom technique [4]. 21

Figure 7: Two layers of a marking menu with four items per layer [48] ........................ 23

Figure 8: Finger-Count Shortcuts: the left hand selects the menu bar's pulldown menu while the right hand selects the item [5]

Figure 9: CountMarks technique: the user presses two fingers to the touch surface; this highlights all the second options in each sub-menu. A two-finger swipe up would select "fish" from the top menu [48] 25

Figure 10: First step of MTPR, selecting target area via sphere-casting technique. 28 
Figure 11: Second step of MTPR, showing selected objects to users. Left) distributed objects inside a quad menu in low density. Right) distributed objects inside a quad menu in high density.

Figure 12: Using fingers to indicate the target object step. All first objects inside each menu are highlighted due to placing one finger on the touch screen. 30 Figure 13: Select the target object by swiping gestures step. The left menu is selected by the user by swiping fingers on the screen to the left direction. 32 Figure 14: Second variation of menu distribution. Objects inside each menu is more than four, so users have to select the desired menu by putting one finger on the screen and swiping it to the right.

Figure 15: Essential equipment used in the experiment. From left, the first image is related to sensor $360^{\circ}$ tracking, the second image is the Oculus Rift with the Samsung Galaxy S8, the third image is another sensor $360^{\circ}$ tracking, and the fourth image is the

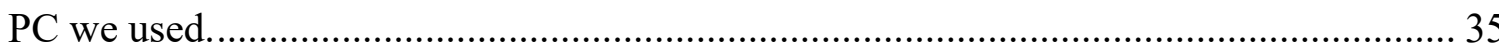

Figure 16: The virtual library scene and the blue book as the target to find. .................. 37 Figure 17: Setup menu shown to participants upon starting the program to enter PID, number of blocks, selection technique, and density.

Figure 18: The scene instruction text box to ask participants to perform the task as fast and as accurate as possible. 39

Figure 19: Desktop scene, low-density virtual library with 425 books in it................... 40

Figure 20: Desktop scene, medium-density virtual library with 1,058 books in it.......... 40

Figure 21: Desktop scene, high-density virtual library with 1,901 books in it............... 40 
Figure 22: Equipment set up on a table, shown in the "setup and experiment instructions" file. 41

Figure 23: Result of two-way ANOVA and Bonferroni-Dunn analysis for book search time. The error bars represent standard error. 44

Figure 24: Result of two-way ANOVA and Bonferroni-Dunn analysis for selection time. This is the average selection time for low, medium, and high density per technique. The error bars represent standard error. 45

Figure 25: Result of two-way ANOVA and Bonferroni-Dunn analysis for error rate. The error bars represent standard error. 46

Figure 26: Result of one-way ANOVA analysis for finger error rate. 48

Figure 27: Selection technique usability and assessment questionnaire. Label numbers indicate the percentage of participants choosing each answer.

Figure 28: IMPReSS technique quad menu, depicting book positions as text (i.e., number of fingers to select a given item in the menu). For example, the Water book is assigned to position 1; touching one finger to the screen and swiping right would select it. 53 Figure 29: Ordering objects inside the quad menu according to IMPReSS technique. Left) creating some predefined spatial positions and dedicated specific menus and position numbers. Right) distributing selected objects according to IMPReSS ordering. 54 Figure 30: Essential equipment used in the experiment. From left, the first image is related to sensor $360^{\circ}$ tracking, the second image is the Oculus Rift with the Samsung Galaxy S8, the third image is another sensor $360^{\circ}$ tracking, and the fourth image is the PC we used.

Figure 31: The blue book is the target to find inside the library's shelves. 58 
Figure 32: Setup menu shown to participants upon starting the program to enter participant's ID, number of blocks, selection technique, and density. .......................... 59 Figure 33: Desktop scene, low density of the virtual library with 1,058 books in it....... 59 Figure 34: Desktop scene, medium density of the virtual library with 1,901 books in it. 60 Figure 35: Desktop scene, high density of the virtual library with 3,055 books in it...... 60 Figure 36: Equipment set up on a table, shown in the "setup and experiment instructions" file. 62

Figure 37: The scene instruction text regarding performing the experiment as fast and as accurately as possible. 63

Figure 38: Result of two-way ANOVA and Bonferroni-Dunn analysis for book search time. The error bars represent standard error. 65 Figure 39: Result of two-way ANOVA analysis for selection time. The error bars represent standard error. 66 Figure 40: Result of two-way ANOVA and Bonferroni-Dunn analysis for the navigation error rate. The error bars represent standard error. 68 Figure 41: Result of one-way ANOVA analysis for finger error. 69 Figure 42: Selection technique usability and assessment questionnaire. Label numbers indicate the percentage of participants choosing each answer. 70 


\section{List of Appendices}

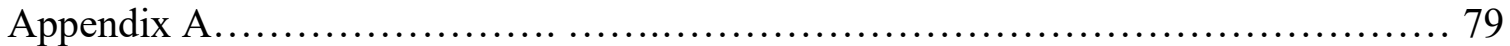

A.1 COVID_19 Screening Form …....................................... 79

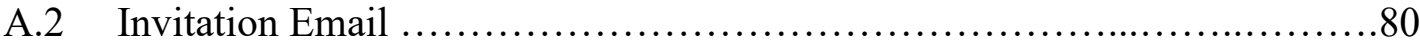

A.3 Contactless Drop-off Email ............................................ 81

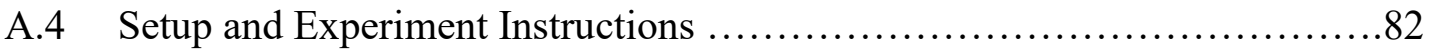

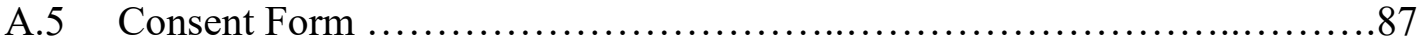

A.6 Pre_Experiment Questionnaire ........................................91

A.7 Usability and Device Assessment questionnaire _ MTPRR and IMPReSS

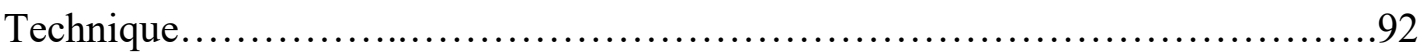

A.8 Usability and Device Assessment questionnaire _ Multi-touch

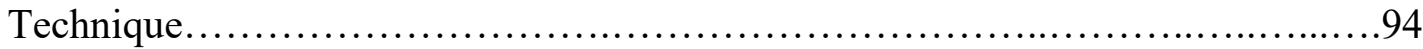

A.9 Usability and Device Assessment questionnaire _ Progressive

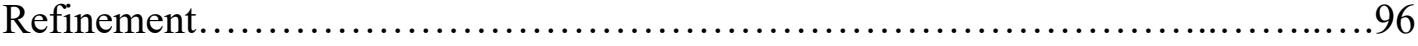

A.10 Post_Experiment Questionnaire........................................98 


\section{Chapter 1: Introduction}

When Virtual Reality (VR) was first introduced to the world in 1968 [68], no one could have predicted its uses today. Recently, the potential impact of VR in everyday life became evident when the pandemic began. VR and Augmented Reality (AR) will shape and play more critical roles in the future in human life. Today, many industries like healthcare, retail, tourism, real estate, education, social and marketing use VR in their experiences $[22,23,33,44,53,63]$.

Improvements to VR hardware and software will generally enhance VR experiences and, more importantly, increase its usability and make it more favored among people as result. For example, recent advances in VR technology have led to more comfortable, lighter, wireless, and more cost-effective head-mounted displays (HMD) such as Oculus Quest2 $[15,43]$. In addition, tracking systems can now track hands and fingers more accurately, which enhances immersion [11] in VR. Advanced development tools such as Unity3D [64] and the Unreal engine [65] are now more accessible for all people by allowing them to create a free account, with many freely available development resources and tutorials.

While VR hardware and development tools are advancing rapidly, VR researchers are focusing on improving the interaction capabilities of VR such as selection, manipulation, navigation, and haptic feedback. Selection is among the most critical tasks since most subsequent operations are dependent on selection. For instance, prior to manipulating an object — and potentially detecting any haptic feedback it provides users must first select the object. Improving selection performance will improve the 
whole VR experience for users, e.g., making the system more usable and potentially improving presence [25] in VR.

Many selection techniques have been introduced to improve selection accuracy and speed [18,20,27,47,49]; however, the 3D selection is still cumbersome [37]. Two key challenges are to create optimized selection techniques that perform efficiently when selecting targets in dense Virtual Environments (VEs) or for remote/small objects.

Progressive refinement is a recent class of optimized selection techniques introduced by Kopper et al. [30]. Progressive refinement breaks the overall target selection task down into sequence smaller selections by opening a quad menu (a square menu consisting of four triangles placed together side by side) at each step. The advantage of these techniques is that users do not need to select the target object precisely in the first step, e.g., with a ray. Instead, they select a cluster of objects in the general vicinity of the target without high precision. Then a quad menu opens, which contains all objects selected in the first cluster, presented on a 2D menu. The user then selects the quadrant containing the target object, refining the selection. The objects on that quadrant get reorganized onto the four quadrants of the quad menu on the next iteration of the selection process. The process repeats until just the target object remains, at which point selection is trivial. Progressive refinement is a fast selection technique, but not in all environments. Previous studies have shown that progressive refinement is not effective in low-density environments [30]. The primary advantage of such techniques is the added precision in high-density virtual environments. Selection of the desired object becomes a sequence of simple $2 \mathrm{D}$ selection tasks $[61,62]$ rather than trying to precisely select an object in 3D in the presence of numerous distractors. 
More recently, Pollack et al. devised a new multi-finger touchscreen-based menu selection technique called CountMarks [48]. CountMarks combined count menus [5], where the user touches a number of fingers to the screen corresponding to the desired item of selection, with marking menus [38], a gesture-based method where a pie menu allows users to select their desired items by swiping their finger in the correct direction (e.g., up, down, right, left). With CountMarks, the directional gesture-based aspect of marking menus was combined with the multi-touch feature of the count menu. Multiple items were shown on each sub-menu of a radial menu. The number of fingers touched to the screen indicates which item inside a menu should be selected, while swiping in a direction indicates which menu should be selected. Consequently, CountMarks is good for situations when the number of items are high since it reduces the number of menus open (the level users need to dig to select the target object). This characteristic decreases the selection time. For instance, if we have three items in each menu and the target object is in the second position, users can place two fingers on the screen and swipe it to the desired menu direction and select the target object, without going one level deeper to the menu.

Another power of CountMarks technique is that it uses the now-ubiquitous smartphone as a VR controller. VR controllers allow the user to interact with and control their actions in VR [67]. They facilitate various interaction techniques, including those for object selection and manipulation or locomotion in the environment. When we use a smartphone as a VR controller, we can make a better VR experience for users. For instance, imagine a user playing a VR game. If their phone rings while they are playing, they have to stop the game, take off the head-mounted display, and answer their phone. 
However, if they could use the smartphone as a controller, they can see who is calling when they are playing and decide whether to answer their phone. Hence, they do not need to stop and break their experience. Previous work has shown that smartphones show promise as VR controllers [2,13]; however, these works are not vast.

Our analysis of related work suggested that progressive refinement and CountMarks are complementary techniques, and we developed a new technique on them. Since this, the directional aspect of CountMarks (for selecting the target object inside the menu) maps well to the quad menu with progressive refinement. Also, the multi-touch aspect of CountMarks introduces the possibility of "short-circuiting" the quad menu hierarchy in progressive refinement by skipping some menu levels for selecting the target object inside the quad menu. On the other hand, careless selection in the first step of progressive refinement with sphere-casting and distributing many selected objects into quad menus is a substantial value that could be enhanced by changing sphere-casting to a directional multi-touch method. In addition, CountMarks uses a smartphone as a VR controller and offers a great opportunity to investigate smartphone performance for selection tasks in VR. Consequently, we decided to combine the CountMarks with progressive refinement and propose a new technique - Multi-Touch Progressive Refinement (MTPR) that uses a smartphone as a controller - to improve previous techniques and solve their problems in this thesis.

\subsection{Contribution}

In our proposed technique of MTPR, we combine the quad menu feature of progressive refinement and the multi-touch gesture-based feature of CountMarks. Based on the rationale above, we developed a smartphone-based application to act as a VR controller 
using MTPR. Users hold the smartphone like a VR controller and can select a cluster of objects in the vicinity of the target. Like with progressive refinement, a quad menu containing the selected cluster of objects with the target object opens. Then users can select the target object by putting an appropriate number of fingers on the smartphone's touch screen and swiping in the desired direction.

We present two experiments evaluating the effectiveness of our technique. In our first experiment, we compared the efficiency of MTPR to conventional progressive refinement and a multi-touch approach (i.e., standard selection method in smartphones). The concept and fundamental approach of these three techniques are very similar, but we hypothesized that MTPR offers faster selection time than progressive refinement and multi-touch regardless of environment density. The rationale behind the hypothesis was that our technique lets users skip parts of the menu selection hierarchy. We also hypothesized that finger error rate (i.e., frequency of incorrect number of fingers on the screen) is high in MTPR for all densities, due to uncertainty about how many fingers should be placed on the touch screen. We compared the techniques in terms of search time, selection time and selection accuracy in different environment densities (lowmedium-high) with the mentioned two techniques.

In our second experiment, we enhanced the MTPR according to the result of the first study. We found that the technique was limited by users being unable to determine how many fingers to put on the screen when swiping. We improved the technique to better show the target object's position in the menu to the user and make it recognizable. This improvement helps to ensure that users do not need to think about how many fingers they have to put on top of the screen and can immediately take action. We also used even 
more dense virtual environments, since a cluttered environment is one of the key factors that affects selection time and accuracy directly. We measured and compared the search time, selection time, and accuracy again between our technique and the other two techniques (progressive refinement and multi-touch) in new densities.

In both experiments, participants completed a selection task in a virtual library, where they had to find a specific book each time. We also asked participants to share their experiences using that interaction technique at the end of each trial. Ultimately, our results show that the unpredictable nature of how many fingers to use in MTPR make users confused and we needed to improve that. We enhanced the technique by ordering items distribution inside the menu and showing objects' positions. After the enhancement, the result of the second study revealed that improved MTPR is a faster and more accurate selection technique than progressive refinement and multi-touch, especially in highly dense environments.

\subsection{Thesis Outline}

Chapter 2 describes background literature along with different types of selection techniques based on task decomposition [37]. We mainly focus on methods that are based on pointing, progressive refinement, counting, and marking menu selection. Chapter 3 describes our design and implementation of the MTPR technique and Improved MultiTouch Progressive Refinement Selection Strategy (IMPReSS). Chapter 4 presents our first experiment and shows the efficiency of MTPR compared to two other selection techniques. Chapter 5 evaluates IMPReSS compared to two other techniques. Finally, chapter 6 discusses our findings and provides discussions, limitations, and future possibilities of IMPReSS. 


\section{Chapter 2: Literature Review}

This chapter describes previous work on selection methods in VR. Selection is a fundamental task in VR and involves the acquisition of a specific object or group of objects from a set [37]. In the real world, selection tasks typically involve picking up one or more objects with the hand but could also involve pointing to them or indicating them via speech [33]. Selection tasks in VR are defined similarly. It is one of the most critical interactions in VR since it is required prior to performing other tasks such as object movement, rotation, and scaling.

Kopper et al. argue [30] that selection methods can be divided into two main categories:

1. Immediate selection, where a target object is acquired after performing a specific action (e.g., touch or point to it and press a button)

2. Progressive refinement, which involves refining a subset of the objects to a smaller group until only the desired object remains.

We briefly describe some immediate selection techniques but mostly focus on progressive refinement techniques [30] since our count-mark menu technique extends this idea [48]. There are many different techniques in each category; we describe only the most relevant ones here.

\subsection{Immediate Selection}

Immediate selection techniques are those that require only one step to select an object. Bowman et al. [36] classify these into different sub-categories based on task decomposition (see Figure 1). 


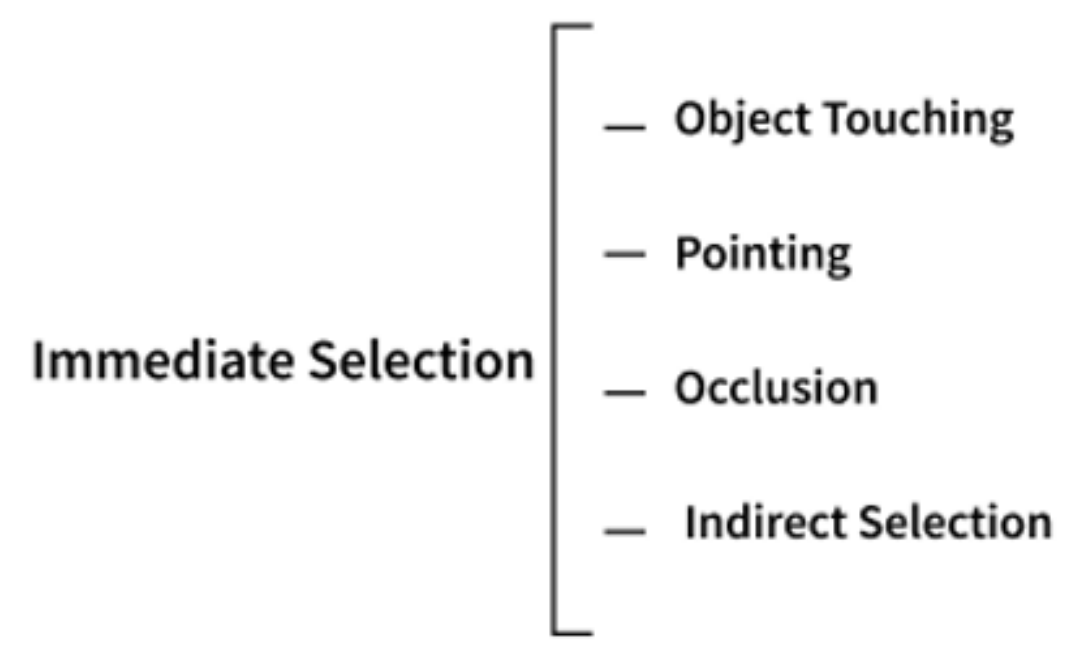

Figure 1: Immediate selection categories based on task decomposition [37].

Task decomposition is based on the observation that all interaction techniques for a certain type of interaction (e.g., travel, selection, or manipulation) require a common set of fundamental elements that serve similar objectives [37]. For instance, in a selection task, the interaction technique should provide the user with the means to imply an object to select, a means to confirm the selection, and a method to deliver some form of feedback (e.g., visual, haptic, or auditory). The advantage of decomposing tasks in this way is that it helps explore the design space of a given type of interaction technique so that new techniques can be "assembled" by merely picking appropriate components for each essential element and putting them together [37].

While there are many VR selection methods, relatively few use a smartphone as a controller for selecting objects in VR $[1,12,26]$. Most techniques using smartphones are not sufficiently fast and accurate for practical usage. We propose a technique employing the best aspects of progressive refinement in combination with the effectiveness of touchscreen interaction, combined with multi-touch marking menus to improve on past 
work in this area. We briefly explain each of the most common subcategories to discuss their key elements.

\subsubsection{Object Touching}

The most natural technique for selecting and manipulating objects in a 3D world is touching or grasping and manipulating objects with the hands. There are two main approaches to object touching: hand-based grasping and finger-based grasping [37]. With hand-based grasping techniques, the user's virtual hand is represented using a singlepoint effector. The most straightforward example is the so-called "virtual hand" technique [37], but other techniques such as Go-Go [49] offer improvements. With the virtual hand technique, the position and orientation of the user's physical hand are tracked and mapped to the pose of their virtual hand in the VR scene [41]. These mappings are usually classified by order of control (i.e., number of integrations) of the tracked position of the user's hand [54,69]. For instance, in zero-order mappings, the input device's displacement changes the position of the controlled element (e.g., cursor or hand). With first-order mappings, the input device's displacement yields a change in the speed of the controlled element. The virtual hand technique is intuitive since it replicates the daily interaction with objects in the real world; however, the main limitation of the technique is acquiring objects out of arm's reach. To select remote objects, users must first move closer to the objects by using a travel technique [37]. Although, travel introduces additional costs in terms of time, complexity, and user attention, and many travel techniques also yield cybersickness [16]. Thus, selection techniques that allow the acquisition of remote objects without travel are attractive alternatives. 
The Go-Go technique is one such attempt to sidestep the travel requirement with virtual hands. With Go-Go, the user can interactively and nonlinearly change the virtual arm length by merely stretching the real hand out. Within a specific distance from the body, the virtual hand moves 1:1 with the real hand like a simple virtual hand technique — beyond this range, it extends nonlinearly with the distance from the body. Previous experiments $[10,50]$ have evaluated the efficiency of selection and manipulation tasks with Go-Go and found that while users understood it quickly, selection efficiency was worse than ray-casting (see section 2.1.2 for more details) since it requires 3 DOF control as opposed to 2 DOF.

Another major limitation of hand-based grasping techniques is they support only a single contact point, effectively losing the fidelity provided by the fingers. Finger-based grasping techniques [37] address this limitation, allowing users to interact with objects with greater precision and supporting new interactions such as twirling a virtual pen between one's virtual fingers. Rigid body fingers [9], soft body fingers [26], and God objects [71] are some examples of the finger-based grasping technique. Rigid body fingers use bend-sensitive gloves, which map the user's real hand and fingers' position to the virtual one represented by a rigid body in a virtual scene. The soft body fingers technique has another approach and uses deformable (i.e., soft body) representations for the virtual fingers and applies a shape-matching algorithm that deforms the virtual fingers' pads to adjust to the shape of the grasped virtual objects dynamically. While using algorithms has improved the efficiency of the soft body finger technique, they are expensive to use [60]. Thus, the concept of God objects is introduced as an alternative. The God object attaches to the virtual object's rigid body and never penetrates it. By 
assigning a God object to a tracked user position, the force direction is calculated when the physical position penetrates the inner space of a virtual object.

All three techniques replicate the fingers and interactions in different fashions. Still, all suffer from the same limitation: without haptic feedback, it is difficult for users to move their fingers to perform the required selection or manipulation. Another issue is the discrepancy introduced between the real and virtual finger positions if virtual fingers cannot penetrate the virtual objects [51]. On one hand, collision avoidance improves such interactions, but on the other hand, if the virtual fingers are in a different position from the real fingers, it may confuse the users.

\subsubsection{Pointing}

Pointing techniques are another fundamental category of object selection and manipulation techniques. With pointing techniques, a vector (i.e., a selection ray) defines the pointing direction and allows the selection of any virtual object intersected by the vector. The selection action occurs when the user issues a trigger, such as pressing a button or issuing a voice command. Pointing techniques were developed to address the main limitation of direct touch techniques, i.e., to make it easier to select objects out of reach by just pointing at them. While there are several different techniques in this class, such as "fishing reel" [10], by far the most common (perhaps in part due to ease of implementation) and well-known is ray-casting $[8,24,41,69]$. With ray-casting, a virtual ray originates from the virtual hand/controller. The direction vector of the ray is controlled by the pose of the virtual hand or device. In situations where it is difficult or impractical to (accurately) track the user's hand, the direction vector can instead be based on the head pose, which is referred to as gaze-based ray-casting [37]. Ray-casting is a 
useful technique, except when high precision is required, e.g., selecting a small object at a far distance; such targets are difficult to select due to a combination of perspective scaling and the small angular motion required [14,28]. Another problem is that raycasting performs poorly in densely packed environments, especially when selecting small/remote objects. In such situations, the performance of ray-casting is limited since hand and tracker jitter is also amplified with increased distance [57].

Several techniques were developed to address this issue, such as flashlight and cone-casting [39]. The pointing direction works the same as standard ray-casting with these techniques, but it replaces the actual selection ray with a conic selection volume. The problem with these techniques is selection ambiguity when more than one object falls within the selection volume. For such cases, researchers defined additional criteria to select the desired object in the selection volume. With flashlight [39], for example, if more than one object falls into the selection cone, the object closer to the cone's center line is selected. If all objects in the selection cone are the same distance to the center line, the object closest to the input device will be selected.

IntenSelect [24] and the bubble [20] cursor solve this issue in different ways. With IntenSelect, objects inside the selection cone are scored based on different frames. With the 2D bubble cursor, a circle dynamically changes its radius to find and touch the closest object to itself. Both techniques are suitable for selecting small or moving objects in cluttered environments, yet they are not fast for all types of environments.

Vanacken et al. [66] extended the bubble cursor for use in 3D environments. The concept of both methods is the same, except in the $3 \mathrm{D}$ bubble cursor, there is a semitransparent sphere as a dynamic ray rather than a circle. The 3D bubble cursor is a kind of 
enhancement of the hand-based grasping technique as well [37]. A limitation of these techniques is that in dense virtual environments, even small movements cause the cursor or ray to resize or snap [2], which increases the selection time and makes the techniques difficult for users to understand.

Other techniques have been devised to improve ray-casting accuracy. One example is the PRISM technique (Precise and Rapid Interaction through Scaled Manipulation) [18]. When a user moves their real hand slower than a specific threshold, PRISM further scales down the motion of the user's virtual hand, improving selection accuracy. Kopper et al. [31] presented the ARM (Absolute and Relative Mapping) technique, which is similar, and involves changing the Control/Display (C/D) ratio and zoom level based on an algorithm defined in the system, rather than the user's distance/angle to the cursor. C/D gain is about showing the amount of movement in a display object. For instance, if a C/D gain is two, it means that one unit of control movement (i.e., mouse) is yielding two units of display movements (i.e., cursor) [46]. However, such techniques can offer high precision by reducing C/D gain, PRISM, and ARM yield with a mismatch of users' real and virtual hand positions. More crucially, they require the user to interact with objects [37] carefully since any fast, slow, or sudden movements can dramatically and inadvertently change the C/D ratio or zoom level. These limitations led researchers to think about techniques that do not require precise selection as the first step. This led to the development of progressive refinement selection techniques. 


\subsubsection{Occlusion}

With occlusion techniques, the user must line up their hand/controller, etc. with the object that they want to select so as to occlude it [1]. Image-plane interaction [47] is an example of an occlusion technique. A major advantage of such techniques is that the user needs to control just two degrees of freedom (2DOF), which makes selection easier [62]. With image plane interaction, users can select and manipulate 3D objects by touching and manipulating their $2 \mathrm{D}$ projections on a virtual image plane located in front of them, rather than directing interaction with the object's 3D model. Although this technique works well for selection, it does not work well for manipulating 3D objects, as their distance from the user cannot be directly controlled since objects are scaled down for manipulation. The limitation of the scaled-world grab technique is that it does not scale well. This matter would make accurate selection and manipulation difficult.

\subsubsection{Indirect Selection}

Indirect selection techniques allow users to select and manipulate virtual objects without directly interacting with them; for example, moving example, moving up and down, or selecting a list of items shown on a TV screen by moving your fingers on your tablet's surface. Simon [56] first proposed indirect touch, where the user can touch an external multi-touch surface to control the display's cursor. Then, with a second finger, they can select a virtual object under the cursor [29]. Once the object is selected, users can manipulate it by one of the indirect manipulation techniques such as Indirect6 [56]. There are several benefits of indirect touch, like interacting with distant objects without requiring travel. Indirect touch also avoids occlusion due to the user's body, which visually blocks interactions, much like the so-called "fat finger" problem [58]. 
Nonetheless, it is still time-consuming to select and manipulate virtual objects [25]. The motion-pointing technique [17] was designed to improve accuracy while keeping selection speed fast by selecting individual objects without pointing at them. With this technique, a specific elliptical motion is dedicated to each potential target. To select an object, users emulate the motion associated with the desired object using the input device. The technique then displays a pie menu depicting the top four known motions that best match the motion performed by the user. The desired object is then directly selected via the pie menu, and thus the number of selectable objects is reduced in this two-step process. However, the technique is not appropriate for environments with many objects since the precision will increase.

\subsection{Progressive Refinement}

Many issues influence VR selection task difficulty, including target size, target distance, and density of objects in the environment. Other issues, like hand/tracker jitter and target movement, are not discussed here but do also impact VR selection tasks [37]. Kopper et al. [30] introduced progressive refinement to address these issues.

Unlike immediate selection techniques, progressive refinement breaks the selection process into several steps. In the first step, the user initially selects a large set of objects, including the target object. The technique then supports the refinement of this initial selection set by progressively reducing the set of selected objects until only the desired one remains. This refinement component provides better control for users by distributing objects into a quad menu and prevents selecting the wrong object (see Figure 2). 


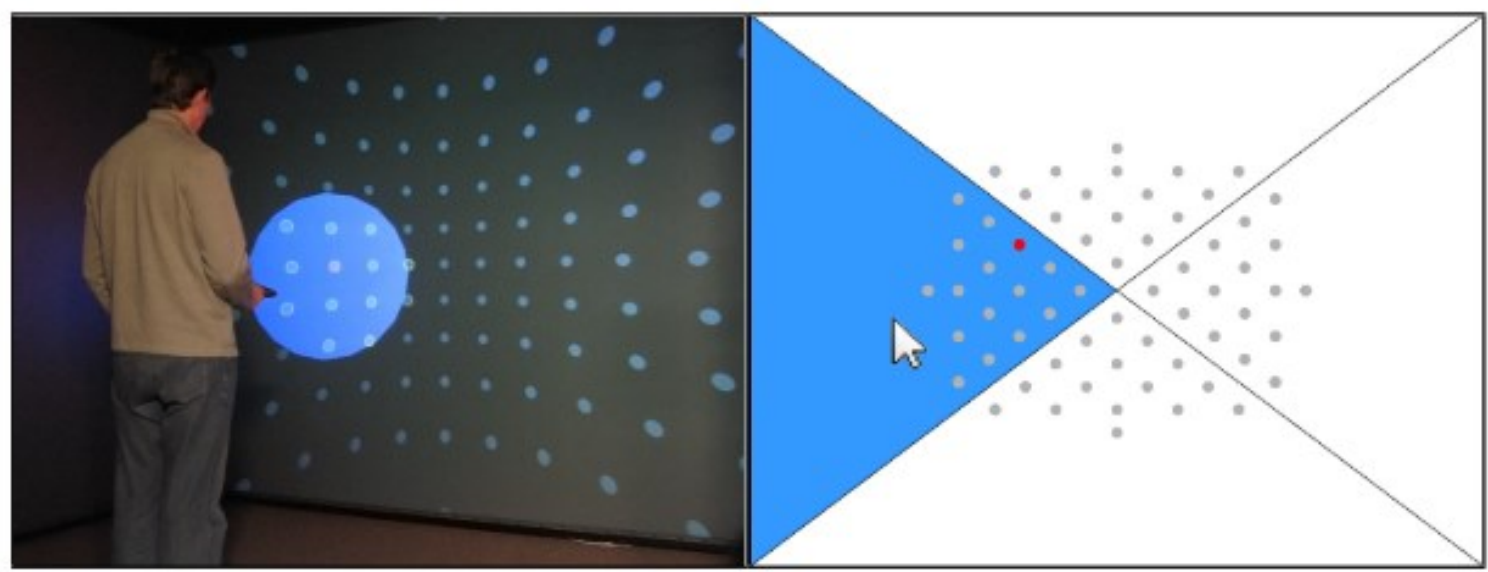

Figure 2: Left) Progressive refinement first step selection; selecting target area. Right) Progressive refinement second step, distributing selected objects inside a quad menu [30].

Kopper discussed three components for progressive refinement. The first is the type of progression, which evaluates selectable objects in each frame, either in a discrete or continuous series of actions. The second is the refinement criteria that define how the user reduces the number of selectable objects. The last component is the method to display the selectable objects. Improvement in any or all of these components improves the overall selection process.

According to Mendes et al. [40], there are two types of progressive refinement: discrete and continuous. There are a few examples of each, as described in the following sections.

\subsubsection{Discrete Progressive Refinement}

With discrete progressive refinement, one or many separate (i.e., discrete) actions happen iteratively to select an object. For example, suppose the user wants to zoom in to see the target object better. In that case, they should repeat the zoom action as many times as necessary to reach the desired zoom level, rather than pressing and holding the trigger button once. The SQUAD technique [30] is one example of a discrete progressive 
refinement technique. SQUAD employs sphere-casting and a QUAD menu (hence the name SQUAD), which reduces the number of selected items step by step to reach the target object. First, users select a set of objects, including the target object using spherecasting, a variation of ray-casting (selects only one object) with a sphere-shaped selection volume on the end of the ray (selects more than one object). All objects inside the selection sphere define the initial "cluster" of selected objects. Then, the selected objects are spread evenly across the four quadrants of a quad menu. The user can then select the target object's desired quadrant using ray-casting (see Figure 2). After choosing the desired quadrant, objects in all other quadrants are discarded from the quad menu in subsequent steps of the selection process. The remaining selectable objects (i.e., those in the selected quadrant) are again re-distributed among a four-quadrant menu. Users repeat this process, reducing the number of selectable objects by three-quarters with each step until only the target object remains. At this point, the selection is trivial.

SQUAD offered significantly better accuracy than ray-casting [37]. Still, it was found to be slower than ray-casting except when selecting small objects or in low-density environments, i.e., where density refers to the number of objects in a unit of space. Highdensity environments have many objects per unit of space in the environment, whereas low-density environments have few objects per unit of space. Higher density environments require more steps through the refinement process, increasing selection time.

One issue with SQUAD is that users lose the original view of the virtual environment during the quadrant menus step since SQUAD replaces the room view with the menu view. Hence, SQUAD may negatively impact presence, the feeling of being 
physically and spatially located in an environment in VR [11]. The Expand technique [12] was proposed as a solution for this problem. Like SQUAD, Expand first selects a group of objects, including the target object, which are placed in the VR scene but employs a different refinement mechanism. With Expand, the user's view zooms to the area they defined in the first step (same VR scene that object were selected initially), rather than opening out-of-context quad menus like SQUAD. Users then can choose the target object using ray pointing (see Figure 3).

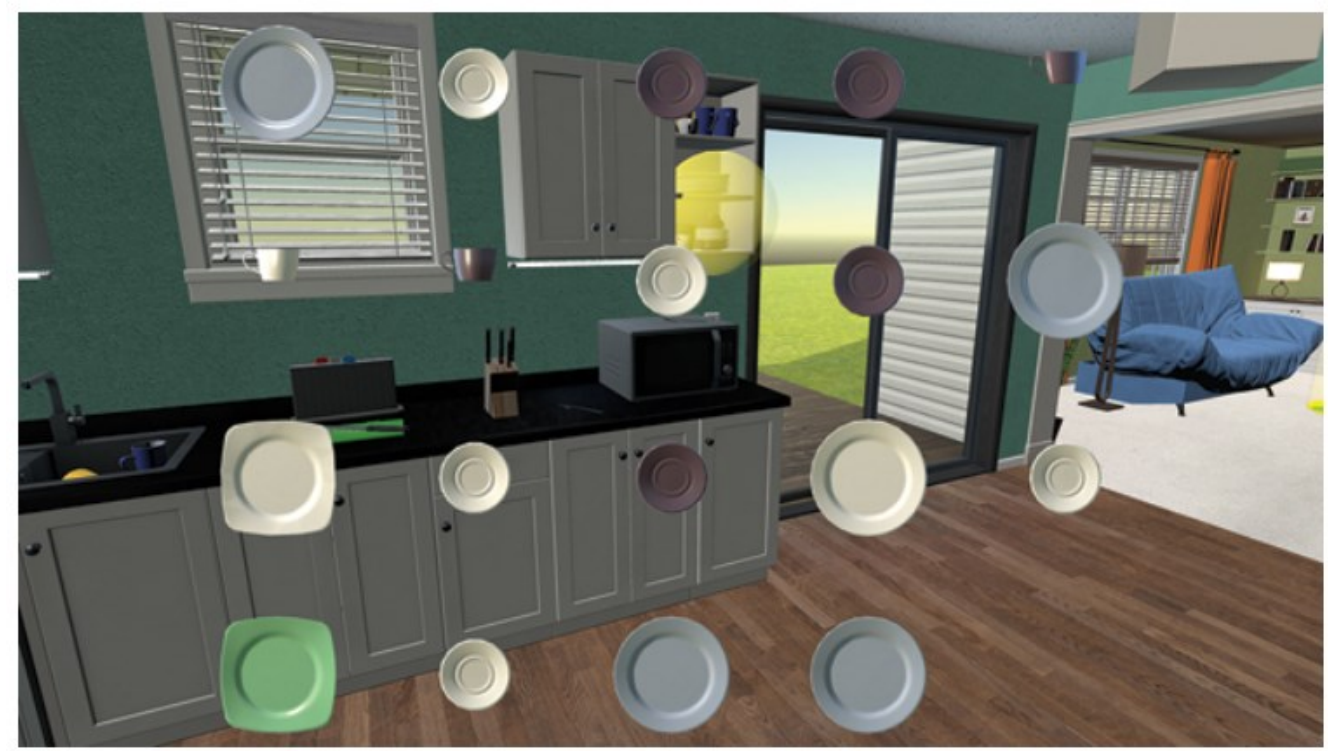

Figure 3: With the Expand technique, selectable objects remain in their original context while clones are displayed in a virtual grid for making a final selection [37].

Cashio et al. [12] compared these techniques and report that Expand is faster than SQUAD, especially in dense environments, but offers lower accuracy. However, neither SQUAD nor Expand perform well in dense environments. A limitation of both Expand and SQUAD is that environments with packed virtual objects put more objects in the initial selection volume. This ultimately increases selection time since the initial refinement menu has more objects in it, necessitating additional steps to refine the 
selection. Based on this observation, Bacim et al. [3] proposed the double bubble technique, which uses a 3D bubble cursor [20] to reduce the number of objects selected in the first step. If there is only a single object in the bubble when the user confirms the selection, it is selected directly. If there is more than one object, all selected objects in the bubble spread into a menu in the image plane, similar to the Expand technique. Both selection steps required by double bubble are fast and do not require high precision, like SQUAD. Double bubble was found to offer better speed and accuracy compared to raycasting.

Other techniques like discrete zoom [4] and PRECIOUS (Progressive REfinement using Cone-casting in Immersive virtual environments for OUt-of-reach object Selection) [40] are both discrete progressive refinement techniques designed for selection accuracy. Discrete zoom distributes the scene into regions like SQUAD's quadrant menu (Figure 4). Users can point to an object to select it directly or point to the target object's region to zoom in, increasing the target size. After selecting the object, the view returns to its original un-zoomed state.
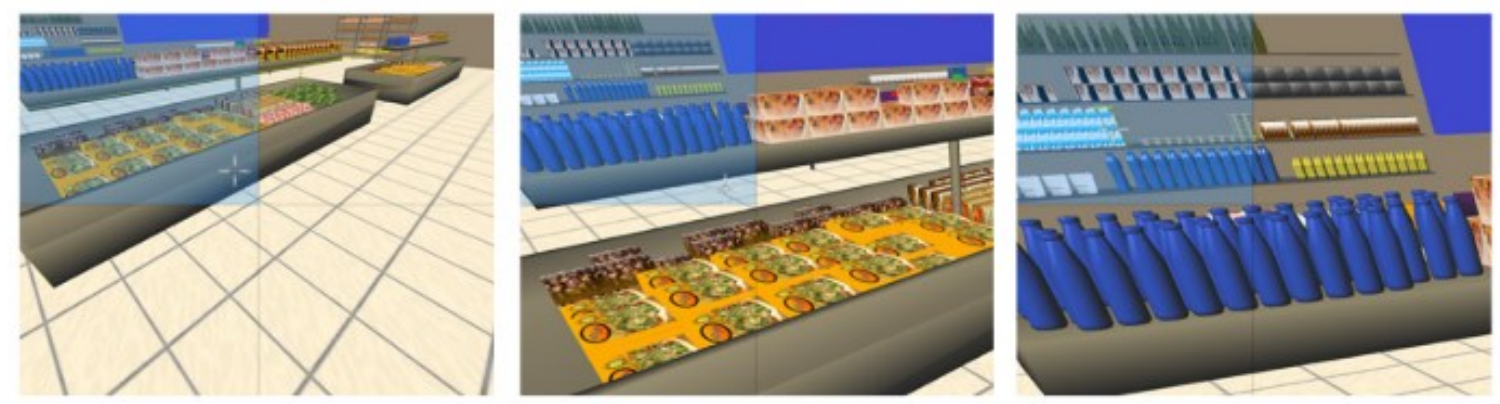

Figure 4: Three refinement steps of the discrete zoom technique [4], left) the first zoom level, middle) the second zoom level, right) the third zoom level.

PRECIOUS uses a selection cone volume cast from the user's virtual hand. Moving closer to the objects offers a more accurate selection (Figure 5). 


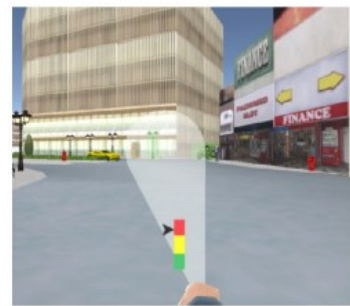

(a)

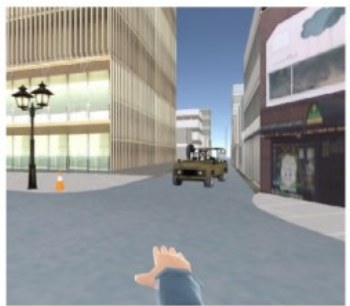

(b)

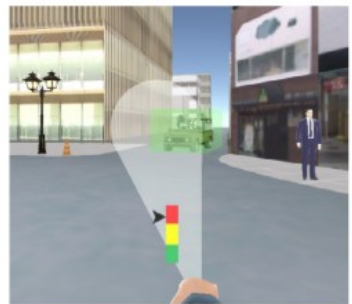

(c)

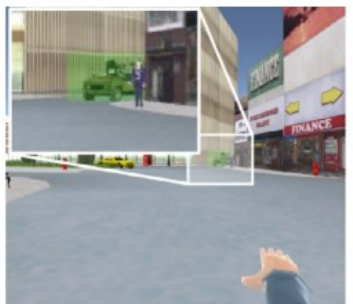

(d)

Figure 5: PRECIOUS technique: (a) selection cone intersecting various objects, (b) refinement phase, moving the user closer to the objects, (c) single object selection, (d) returning to the original position with the object selected [40].

With both techniques, the desired location is zoomed in to the user, whether by moving the scene closer to the user (discrete zoom) or the user closer to the scene (PRECIOUS). Unlike SQUAD, the quadrants menu is always visible (and transparent) with discrete zoom, preserving user presence. Both techniques achieve high accuracy by sacrificing speed. According to previous studies, providing both good speed and high accuracy with one technique requires consideration of the target size and the environment density [4]. This is because these are two important factors that affect selection speed and accuracy the most. For example, it is more time-consuming to find a tennis ball than a football ball in a virtual store since it is smaller. Therefore, while SQUAD works well for selecting tiny targets, the zoom techniques may work better in cluttered environments, while Expand is faster than discrete zoom [34].

\subsubsection{Continuous Progressive Refinement}

Unlike discrete progressive refinement techniques, continuous progressive refinement techniques use one or more continuous actions to acquire the target object. For example, continuous zoom [4] is similar to discrete zoom, except instead of spreading the scene to a quadrant menu, it zooms to the cursor area continuously and constantly (Figure 6). 

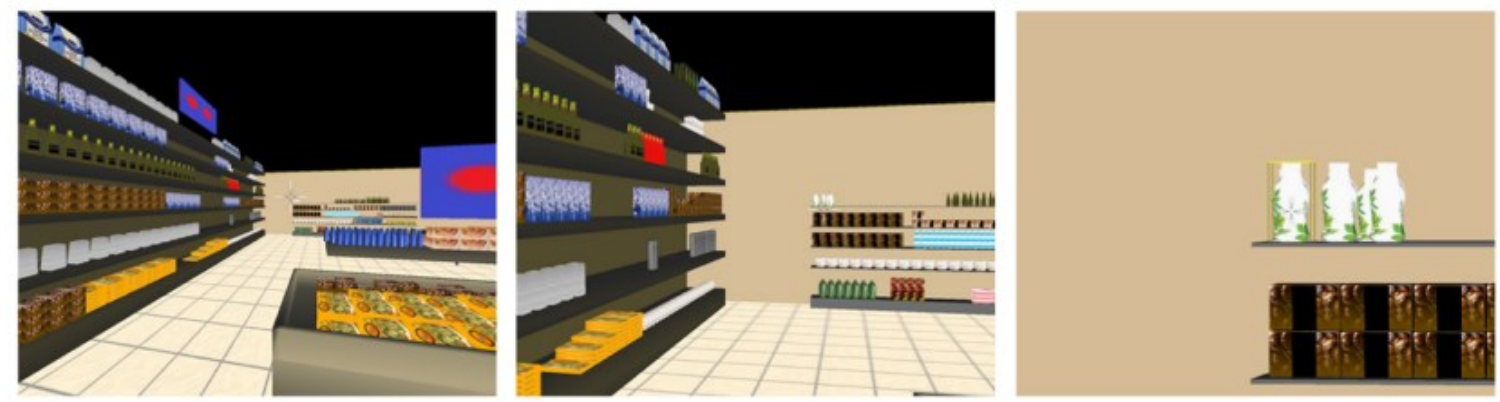

Figure 6: Three snapshots of different zoom levels achieved with the continuous zoom technique [4].

With the intent-driven selection (IDS) technique [45], users manually adjust the selection sphere volume and zoom in and out into the environment by changing their hand and finger position, making the sphere smaller or bigger until it reaches the target object. However, selecting objects far from arms-length is difficult because of the physical constraints of real hand gestures and positioning.

Other techniques that are similar in concept are based on ray-casting [8] or conecasting [40]. Flower ray [21] is one example, which is the enhanced method of depth ray and lock ray [21]. With flower ray, when the user wants to select an object, the items intersected with the pointing ray are selection candidates. The user then presses and holds a button first to lock the point and then to distribute the intersected items into a marking menu (see section 2.3 for details). Depth ray is faster than flower ray and lock ray; however, they offer comparable accuracy. However, none offer high selection speed in dense environments.

Shadow cone-casting [57] was designed for group selection, but it did not solve the selection speed issue. Shadow cone-casting works like flashlight, except there are no pre-defined rules for selecting objects inside the cone. With this technique, when a group of objects is contained in the selection cone, the user can press a button and move their 
hand to reduce the number of selected objects. Whenever users release the button, all objects inside the cone will be considered as target objects.

The disambiguation canvas [14] is another method based on progressive refinement, which uses a mobile device as a controller. This technique uses two steps. First, the user selects the target object's region by pointing the mobile device into the area and using a volume casting technique. Second, a rectangle appears in front of the user by touching the screen, rearranging the selected object in a matrix form. The user can then select the desired object by touching the screen to move a cursor. Such a technique is potentially convenient due to the familiarity of mobile devices. However, the technique does not offer fast selection since many objects in the cone make the selection task difficult.

\subsection{Marking Menus and Count Menus}

A marking menu [36] is a selection technique that allows users to select items from a radial/pie menu in the desired menu's direction. For example, the menu seen in Figure 7 is distributed into four items. A menu with more than four items, with only four items per "layer" of the menu, means users must navigate to a deeper layer of the menu to select the desired item (as seen in Figure 7). Research on marking menus has shown that eightitem menus are the practical upper limit to avoid accuracy problems. This reduces the number of depth layers of the menu but decreases the accuracy of selection at any given layer. 


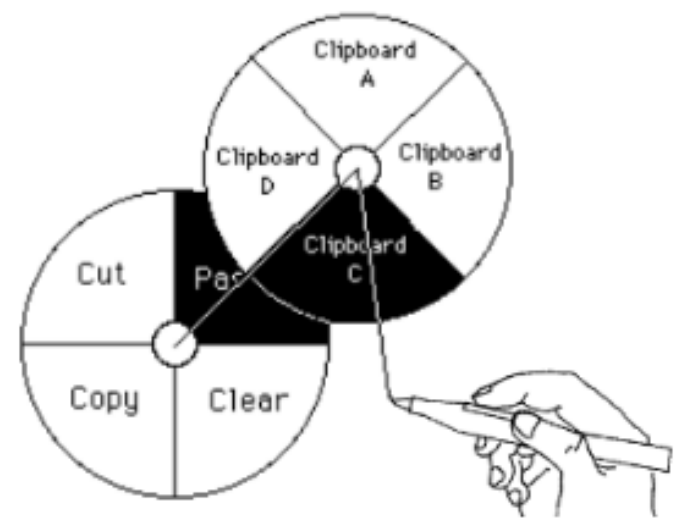

Figure 7: Two layers of a marking menu with four items per layer [48].

Marking menus support swipe gestures for faster and more accurate selection than the linear selection menus [7]. One reason is that the display of the menu does not delay the user; a user familiar with the layout of the menu can simply perform the gesture (i.e., the "mark") required to reach a given item in a sub-menu, without waiting for the menu to visually appear. Unlike linear menus, marking menus can be operated "eyes-free" since selection is based on movement direction, not the menu item's position [35].

The problem with marking menus is when there are eight items or more at levels greater than two, which increases the error rate [34]. According to Kiger et al. [28], users perform better in selecting single-level menu items even with many objects than selecting multi-level menus since the users get lost while drilling down into multi-level menus to reach the desired target. Therefore, different marking menu variations such as the flower menu [6] and polygon menu [70] were developed to increase menu breadth, which can increase selection speed and accuracy.

Lepinski et al. [38] proposed a method to increase menu breadth using multitouch input. In this method, users put their fingers in different combinations, like guitar chords, on a touch screen. As a result, different menus pop up, and the user can select the 
desired menu by swiping in the desired direction on a large touch screen. Their experiment revealed that the simple subset of finger combinations is faster than traditional marking menus, although most combinations are complicated for users to perform [48].

The count menu [5] is a bimanual selection technique that lets users select a menu and items from the menu based on how many fingers are touching the surface. For example, in Figure 8, the user employs their three fingers of the left hand to select the menu on top (form menu) and their four fingers of the right hand to select the fourth item inside the menu (Ellipse).

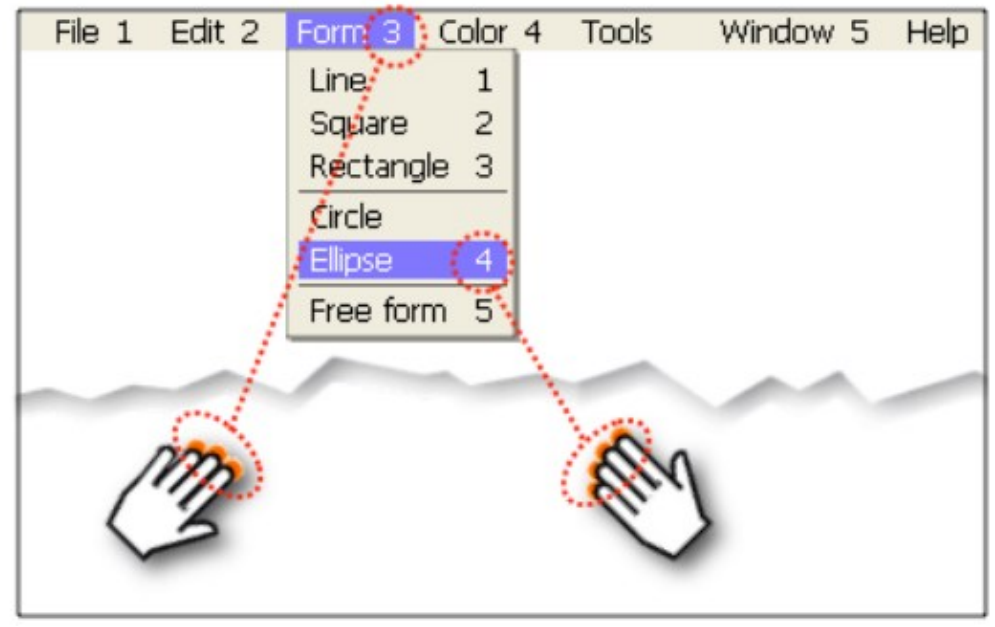

Figure 8: Finger-Count Shortcuts: the left hand selects the menu bar's pulldown menu while the right hand selects the item [5].

One hand selects the menu, while the other hand chooses items from that menu. Thus, users can choose from up to five menus with up to five items each. According to Lepinski et al. [19], the count menu is easy to use and learn. It is also faster than the 3D in-air marking menu [52] with the same accuracy [32]. Pollock et al. [48] combined the multi-touch feature of count menus with the swipe gestures of marking menus to develop the CountMarks menu. CountMarks provides more menu items in fewer sub-menu layers 
than a marking menu. The fingers of only one hand are required to select the items, unlike the count menu. The number of fingers the user touches to the touchscreen indicates the item inside each menu. Swiping in a given direction will select the chosen item from the menu in that direction. For instance, in Figure 9, by putting two fingers on the screen, the second options in all eight menus are candidates for selection. By then swiping to the right, the item "Orange" will be chosen from the fruit menu (i.e., the menu on the right-hand side, or three o'clock position).

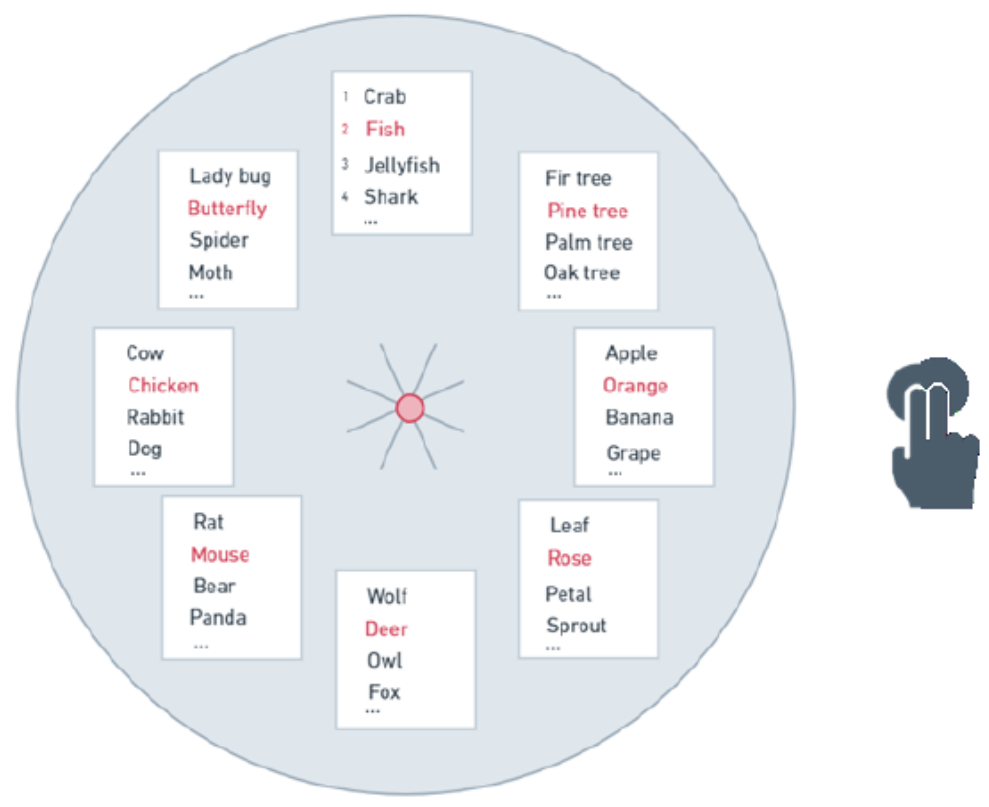

Figure 9: CountMarks technique: the user presses two fingers to the touch surface; this highlights all the second options in each sub-menu. A two-finger swipe up would select "fish" from the top menu [48].

Other research has shown that marking menu navigation is not easy, and users may be lost in the hierarchy [28]. Broader (rather than deeper) menus like CountMarks offer an easier way to find a desired item [55]. Also, results of the Pollack et al. study [48] showed that CountMarks is faster than the count menu and marking menu. However, 
CountMarks offers worse selection accuracy than conventional marking menus due to the possibility of making mistakes with the number of fingers on the screen.

\subsection{Summary}

To summarize, there are many different VR selection methods, with progressive refinement being one of the most accurate ones. Yet, despite offering good accuracy, such techniques do not offer high speed, especially in densely packed environments. We want to design a technique to address remote selection in densely packed environments to offer high precision and low selection time by using existing progressive refinement techniques. The CountMarks menu technique offers potential improvements here by increasing menu breadth and potentially allowing quicker selection (based on how many fingers are touching the screen) rather than digging down into a menu. Therefore, we propose to combine CountMarks with progressive refinement to select objects, to initially select objects in a group, and then use CountMarks menus to choose items inside the menu. We argue that this should improve progressive refinement techniques' speed without reducing accuracy in dense environments. We describe this technique fully in the next chapter. 


\section{Chapter 3: Design and Implementation of Multi-Touch Progressive Refinement}

Selection speed and accuracy are two key factors influencing selection efficiency and have always been a challenge for VR researchers. We designed the multi-touch progressive refinement technique to improve selection speed while offering precise accuracy. We present two versions of the technique: a first version, MTPR, evaluated experimentally in this chapter, then an improved version, IMPReSS, enhanced based on the results of the first evaluation.

\subsection{Design and Implementation of MTPR}

Similar to conventional progressive refinement techniques, the technique involves several distinct steps to accomplish the selection task. Each step is described below.

\section{Step 1: Selecting Target Area}

Like SQUAD and other progressive refinement techniques, users do not need to be precise in the first step of selection. Users first select the general area of the target, the same as other progressive refinement techniques. This employs ray-casting, with a selection sphere at the intersection of the ray and scene. Objects within the sphere are deemed selection candidates. See Figure 10. 


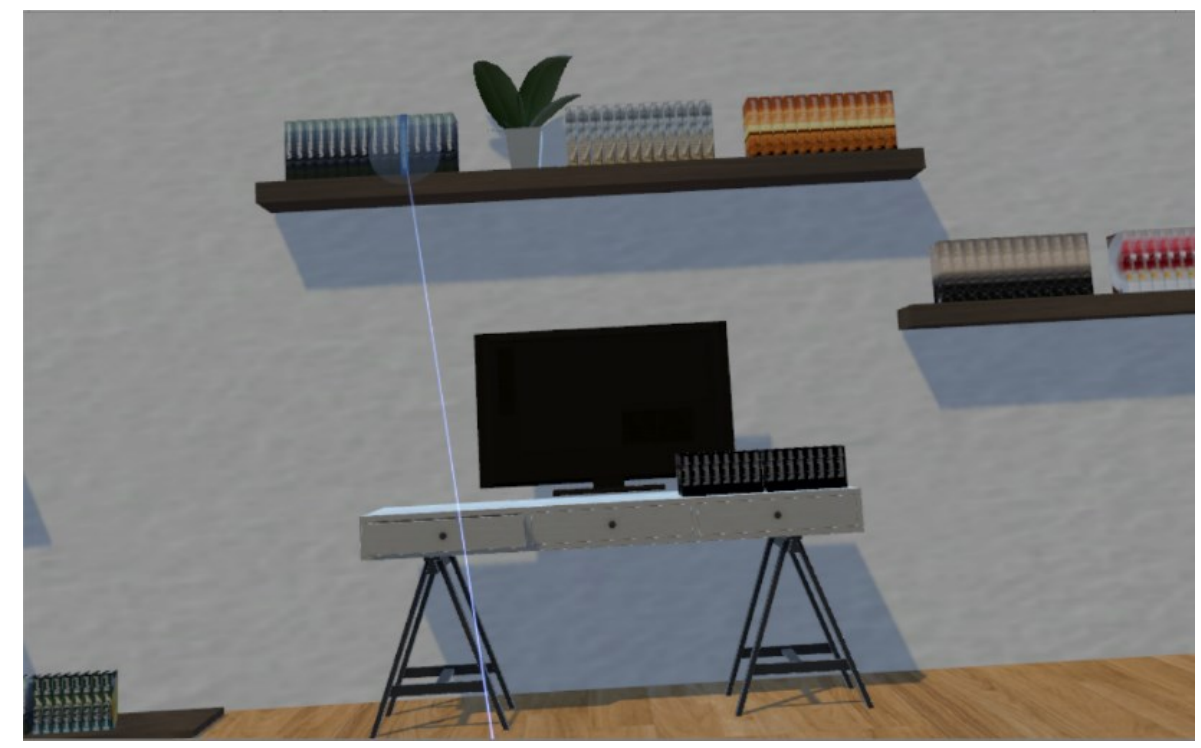

Figure 10: First step of MTPR, selecting target area via sphere-casting technique.

\section{Step 2: Showing Selected Objects to Users}

After selecting the target area, multiple objects are initially selected. Then, we distribute all selected objects inside a quad menu, similar to SQUAD. Objects are randomly distributed (same as progressive refinement) to each quad menu. For the right and left menu, we place the objects from top to bottom, and for the top and bottom menu, objects are placed from left to right. We start arranging objects from the outside of each triangle and continue to the middle of that. The number of objects inside each quadrant of the quad menu depends on the environment density (i.e., virtual environments with more densely packed objects will tend to result in more objects in the initial selection). If the density was low, objects inside the menu were bigger and closer to the center of the triangle. Also, horizontal and vertical spaces between objects were more. However, when the density was high, objects inside the menu were smaller and placed closer to the edge of triangles. In addition, spaces between objects were less, so we could put more objects inside the menu. For example, Figure 11 (left) depicts the object distribution inside the 
quad menu for a low-density environment, while Figure 11 (right) depicts the initial selection in a high-density environment. The maximum number of objects inside each menu is 16 (maximum number of menu items in marking menu technique was eight) according to the size of each quadrant menu, size of each object inside the quadrant menu, and spaces between them. Hence, we can support up to 16 (objects) * 4 (number of menus) $=64$ objects in each menu level.
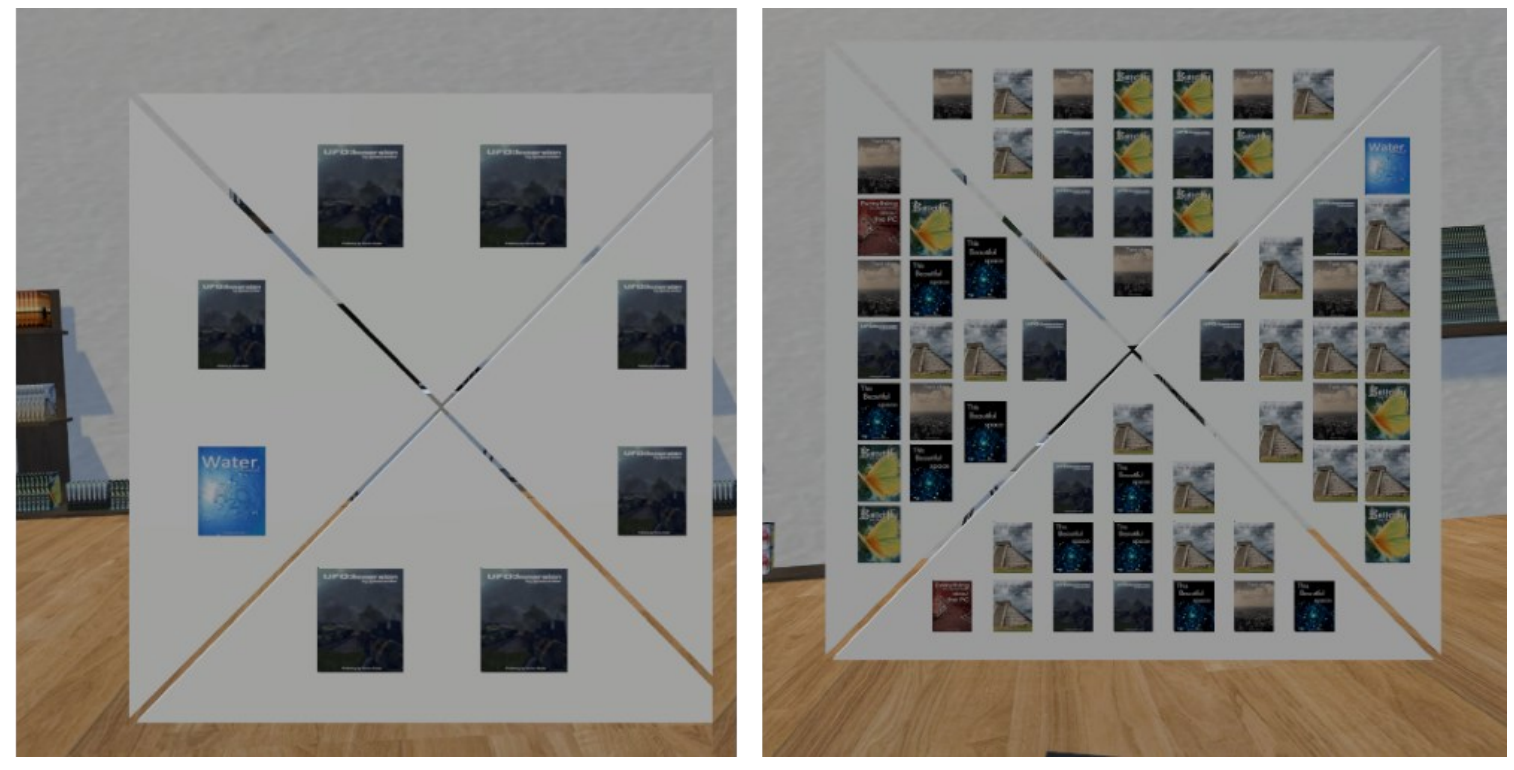

Figure 11: Second step of MTPR, showing selected objects to users. Left) distributed objects inside a quad menu in low density. Right) distributed objects inside a quad menu in high density.

\section{Step 3: Selecting a Target Object Inside the Quad Menu}

Assuming the desired target object was in the initial selection, the user must next find the target object inside the desired quad menu in order to select that. If the desired object was not inside the initial selection, the menu would not open and users must repeat the initial selection until it is. While the preceding steps of our technique are similar to other techniques [28], this step is the main difference in our technique. Thus, we break down this step into sub-steps to explain it clearly. 


\section{Step 3.1 Using Fingers to Indicate the Target Object}

After finding the desired object in the quad menu, users can select it by touching the smartphone screen with the appropriate number of fingers related to the target object's position. The technique supports up to a maximum of four fingers. It does not matter which finger (thumb, index, etc.) of which hand (right or left) is touching the surface in determining this count. As the user touches the screen, the current selection candidate object related to the number of fingers placed on the screen is highlighted in yellow. This helps users understand which item will be selected. For instance, in Figure 12, the target object is the blue book, which is in the first position. When users put one finger on the touch screen, all objects in the first position inside each menu are highlighted to yellow as guidance, indicating they will be selected if users swipe their fingers in that direction.

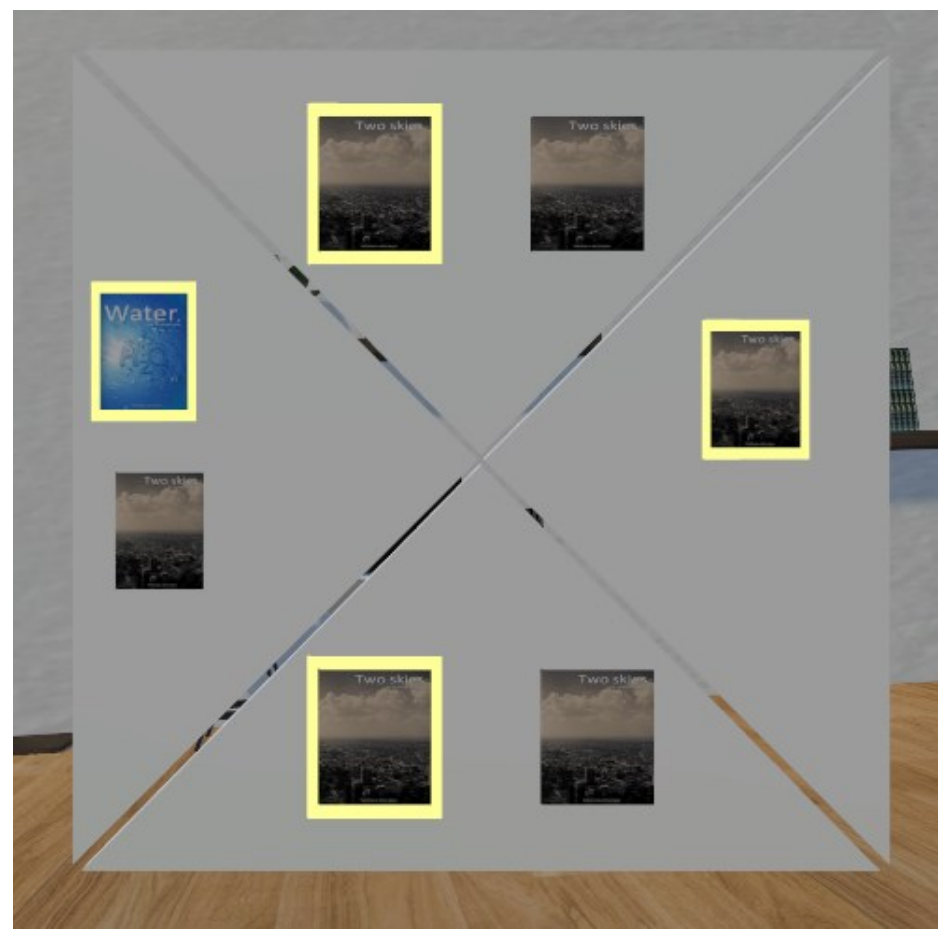

Figure 12: Using fingers to indicate the target object step. All first objects inside each menu are highlighted due to placing one finger on the touch screen.

\section{Step 3.2 Select the Target Object by Swipe Gesture}


After indicating the desired object by touching the screen (as described above), users can finalize their selection by swiping their fingers touching the screen in the desired direction. As soon as the user moves their fingers in the desired direction, the related menu is highlighted in green to help users see which direction they are moving. For example, in Figure 13, the blue book (target object) is in the left menu, in the first position. To select it, the user can put one finger on the screen, which results in the book being highlighted yellow. Swiping their finger in the left direction highlights the left menu green highlight. The selection is completed when the user swipes their fingers with a specific distance, which is entirely to the edge of the touchscreen through the desired menu quadrant. We believed that swiping to the edge is a common action that most people perform with their phone on a daily basis (i.e., swiping to the edge to answer the phone, swiping to the edge to delete a text message), so it helps them to interact with technique easier. Since we are using a smartphone (with a display of 5.8 inches) in our technique, swiping to the edge will not be a problem. However, in the future if we want to test our technique with bigger touch screens like 20 inches, we need to redesign this part and complete the selection task by a flick gesture.

At present, the technique supports only the four primary swiping directions (same as the number of menus in the marking menu technique). However, this could be extended to support more (e.g., up to eight) directions and menus, and to display more selected items, or to distribute items further across each menu. 


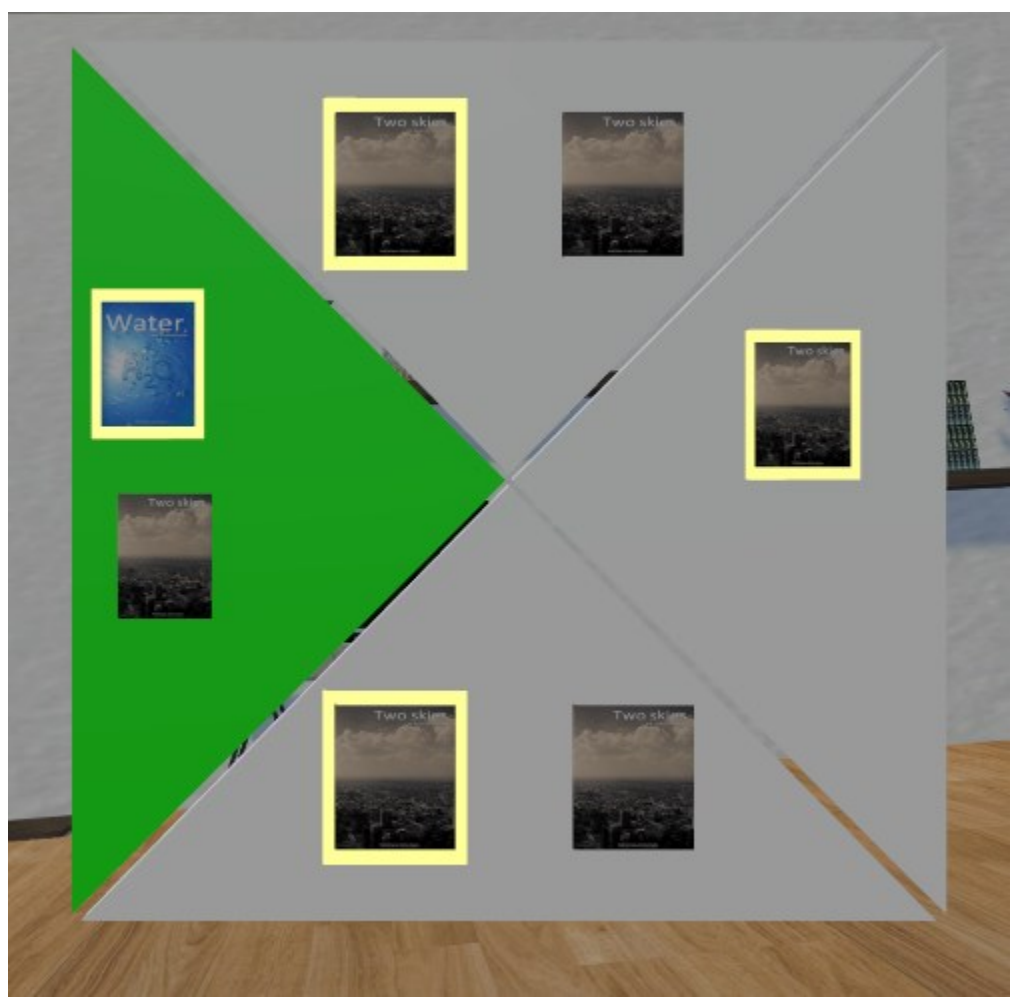

Figure 13: Select the target object by swiping gestures step. The left menu is selected by the user by swiping fingers on the screen to the left direction.

\subsection{Menu Distribution Variations}

When selected objects distribute into a quad menu, two variations happen:

1. When the number of selected items inside each menu is less than four, users can easily select their desired object, as described in sections 3.1 and 3.2 (Figure 13), in one step.

2. When the number of selected items inside each menu is greater than four, users need to go into a deeper menu (which we refer to as "levels" of the menu) to be able to select the target object (to further refine the selection). To go to a deeper menu level, they need to put one or more fingers on the touch screen and swipe it in the desired quad menu direction. See Figure 14. Since there are more objects in the quad menu than the user has fingers, none of the objects highlight in this 
situation; this step is only used to further refine the selection. After that, the menu items from the selected quadrant redistribute, containing items inside that specific menu in the fashion seen in Figure 11 (left). They repeat this process until there are four or fewer objects inside each quad menu. They can then select the object as explained in sections 3.1 and 3.2.

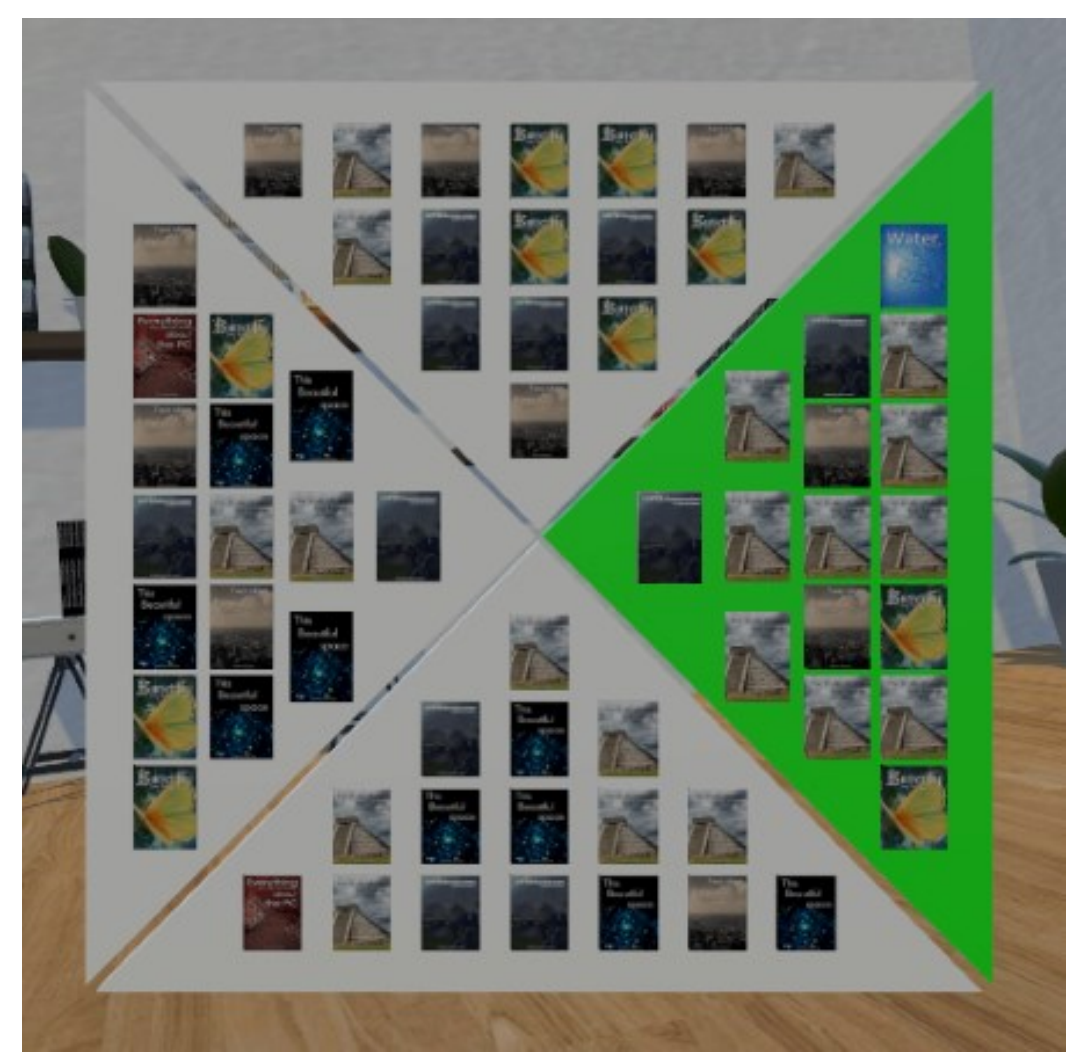

Figure 14: Second variation of menu distribution. Objects inside each menu is more than four, so users have to select the desired menu by putting one finger on the screen and swiping it to the right.

\subsection{User Study 1: Performance Evaluation of Multi-Touch Progressive}

\section{Refinement}

We conducted a user study to compare the speed and accuracy of selection using MTPR to the SQUAD progressive refinement technique and multi-touch technique. We chose SQUAD and multi-touch to be compared with MTPR since all three techniques operate 
similarly in the first and second steps (selecting the target object from the VR scene and distributing selected objects into a quad menu). However, they have some fundamental differences in the selection process for the objects inside the quad menu. Our hypothesis was that MTPR offers lower selection time than progressive refinement and multi-touch for all densities. We also hypothesized that finger error rate is high in all densities in the MTPR technique. The reason for the second hypothesis was the uncertainty over the number of fingers participants have to put on the screen to select the target object.

\subsubsection{Participants}

We recruited 12 participants ( 5 male, 7 female). Participants were between 30 and 46 years old $(\mu=33.6, S D=5.9)$. We surveyed their level of experience with VR systems:

$58.3 \%$ had some VR experience before (used VR 4-5 times overall), while $41.7 \%$ had no VR experience so this was their first time using a VR system. None of the participants were expert VR users (use VR at least three times per week). All participants were righthanded and had normal or corrected-to-normal stereo vision, assessed based on questioning during recruitment.

\subsubsection{Apparatus}

This section describes the hardware and software we used to perform the experiment.

\subsubsection{Hardware}

We conducted the experiment using a PC with an Intel Core $17-7700 \mathrm{~K} \mathrm{CPU}$ at $4.20 \mathrm{GHz}$ with 32 GB of RAM, with an Oculus Rift CV1 head-mounted display and two-sensor $360^{\circ}$ tracking. A Samsung Galaxy S8 (OS Android 9.0) was used in this experiment as the VR controller. Figure 15 depicts the hardware used in the study. Due to the COVID19 pandemic, we delivered this hardware to the participants via a contactless drop-off 
protocol. Other hardware such as the monitor, keyboard, and mouse were expected not to influence the experiment, and thus participants used their own devices.

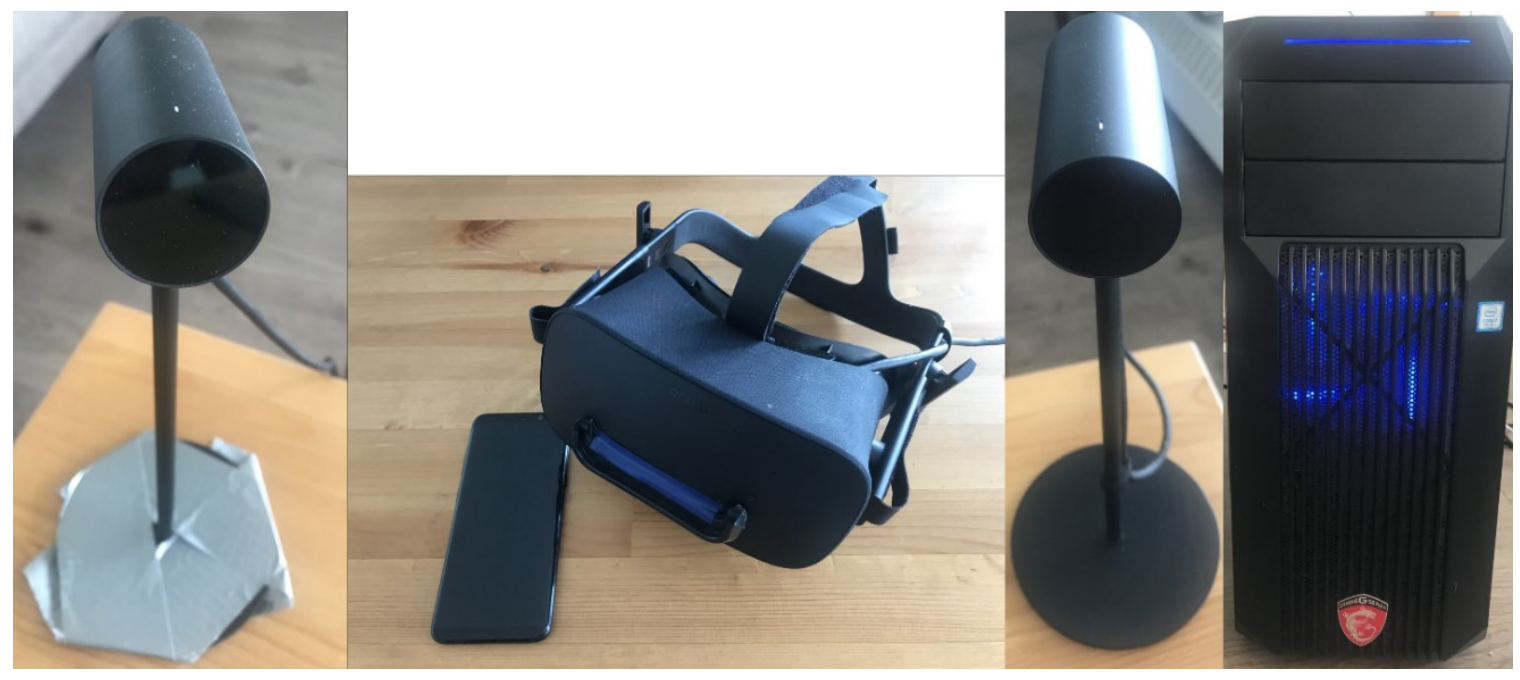

Figure 15: Essential equipment used in the experiment. From left, the first image is related to sensor $360^{\circ}$ tracking, the second image is the Oculus Rift with the Samsung Galaxy S8, the third image is another sensor $360^{\circ}$ tracking, and the fourth image is the $\mathrm{PC}$ we used.

\subsubsection{Software}

We developed two applications using Unity 3D. To make the smartphone operate as a VR controller, we needed to access the phone's sensors. To do that, we used the PhotonView application [46], a client-hosted networking for Unity. We made the desktop Unity application act as a server, and the smartphone Unity application act as a client that connected to the server upon starting. We used the client/server model to transmit the phone's gyroscope data such as navigation and rotation axis. The software started reading the gyroscope data as soon as the server and client connected to one another. We used the smartphone gyroscope data to duplicate the same motions and rotations of the smartphone in the user's hand into the desktop VR scene. In this fashion, we could make 
the smartphone act as a VR controller. This supported the 3DOF orientation of the device, sufficient for ray-casting.

On the server side, we designed a virtual library scene with some bookshelves and a table with a TV on it. We also added some books to the shelves and some small sized books on the left and right sides of the TV. Users were tasked with finding and selecting the blue book, as shown in Figure 16. The book was positioned pseudo-randomly on the library shelves. The software also presented the quad menus during the selection process.

In the client environment, we designed an empty scene with a ray that had a sphere at the end with a fixed diameter in all densities and techniques to select objects from the shelves by the ray-casting technique. We replicated the client's ray into the server and showed that to the user. Changing the position or orientation of the smartphone updated the ray in the desktop scene accordingly. To select books from shelves, the user had to first find the book by looking around the environment; then, they had to point at the desired book by moving the smartphone and finally touch the smartphone's screen. After that, a quad menu opened for them to select the target object. If users selected a wrong menu or wrong book inside the menu, no other menu opened for them, and they could not go to a deeper level to avoid losing any time. 


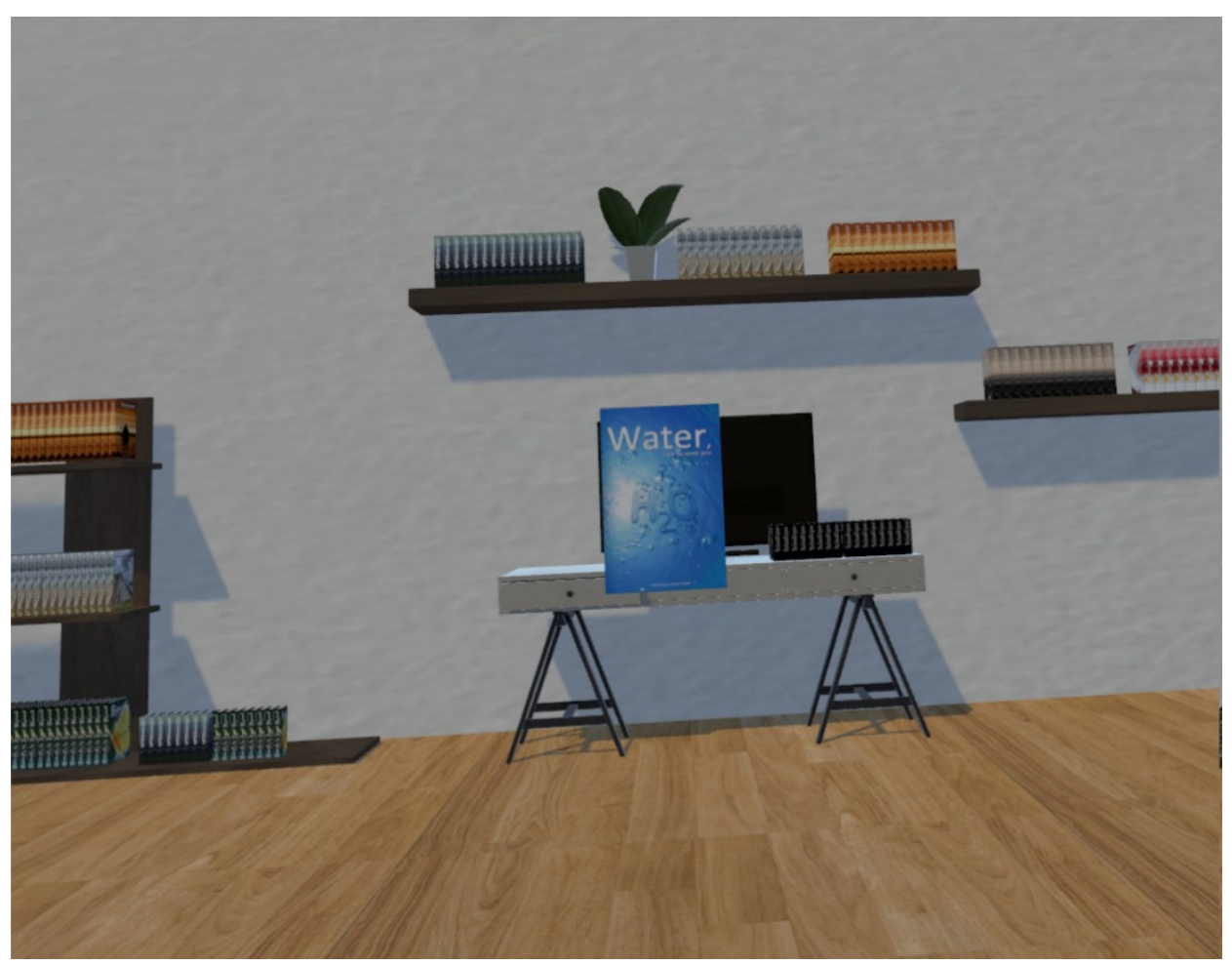

Figure 16: The virtual library scene and the blue book as the target to find.

We also developed multi-touch and SQUAD techniques to be able to compare MTPR with them. Multi-touch technique implementation had two parts. The first part was related to finding and selecting the target book, which was exactly the same as MTPR. The second part was selecting the target book from the menu, which was similar to MTPR's second version of menu distribution when the number of objects inside the menu is more than four. When the quad menu opened, participants had to put their fingers on the touchscreen (no matter how many fingers) and swipe it to the desired menu direction. They had to repeat this action till only the target book remained on a menu. The SQUAD technique [30] was the last technique we implemented, which was described in section 2.2.1.

Upon starting, the software presented a start-up screen requesting the participant's ID number, number of blocks, and experimental conditions, including the selection 
technique and environment density (see Figure 17). The software also recorded the time users spent to find the target book (search time), the time required to select the target book from the menus (selection time), and the number of errors they made in selecting the right book and/or right menu (error rate).

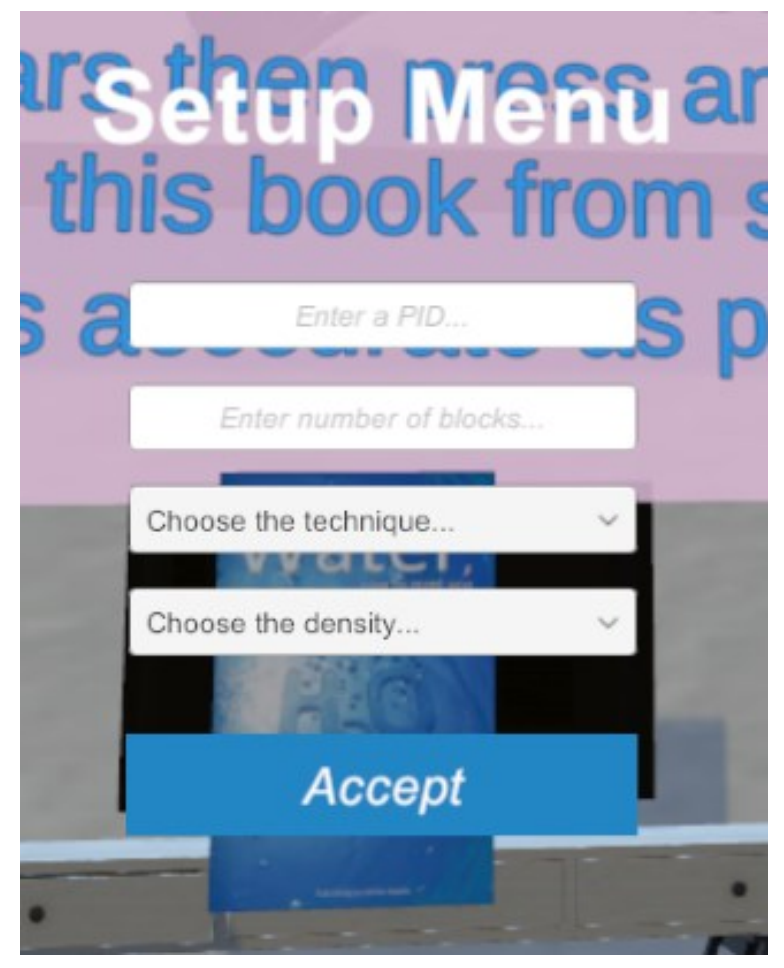

Figure 17: Setup menu shown to participants upon starting the program to enter PID, number of blocks, selection technique, and density.

Upon starting the task, a message was shown in the scene, which provided instructions to participants on how to continue the experiment (see Figure 18). To advance to the next trial (which would randomly put the book somewhere else), participants had to press any key on the keyboard after each time selecting that. Upon pressing any key, the timer started to count the task completion time. Upon pressing a key after the last selection trial, the experiment ended. 


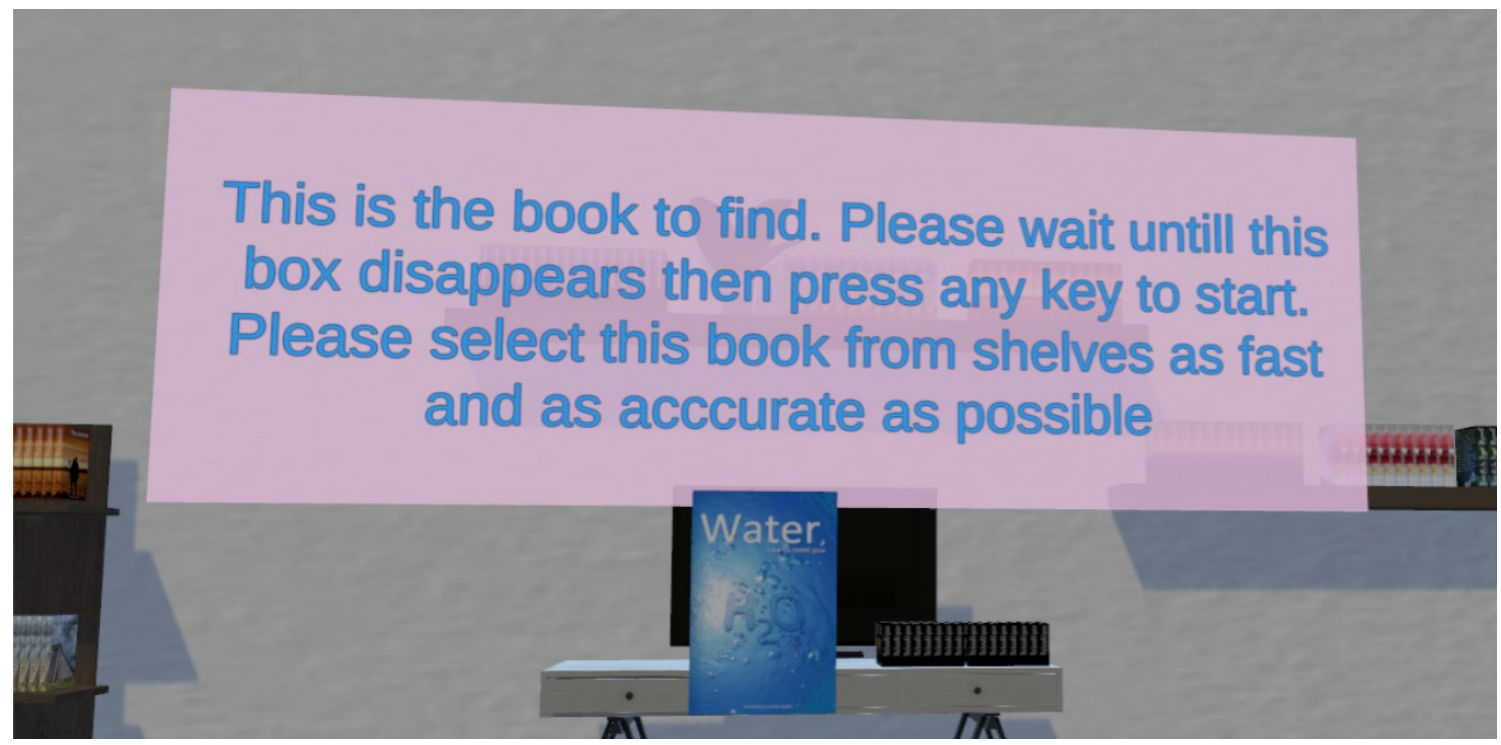

Figure 18: The scene instruction text box to ask participants to perform the task as fast and as accurate as possible.

The books on the library shelves were presented at varying levels of density. To design the density, we considered the maximum neck rotation $\left(90^{\circ}\right.$ of rotation to both sides and approximately $80-90^{\circ}$ of flexion) [59] for humans. So, we put one shelf on the right side (needed approximately $80^{\circ}$ rotation from the centre) and one shelf on the left side (needed approximately $80^{\circ}$ rotation from the centre), which contained different rows of books. Then we put up three more shelves and one table with a TV on it between the right and left shelves. Accordingly, we defined three different density levels: low, medium, and high. We will explain the details of setting books inside shelves next.

Low density: This included four rows of books on three shelves and one row of books on two shelves. At this density, there were a total of 425 books in the whole scene. See Figure 19. 

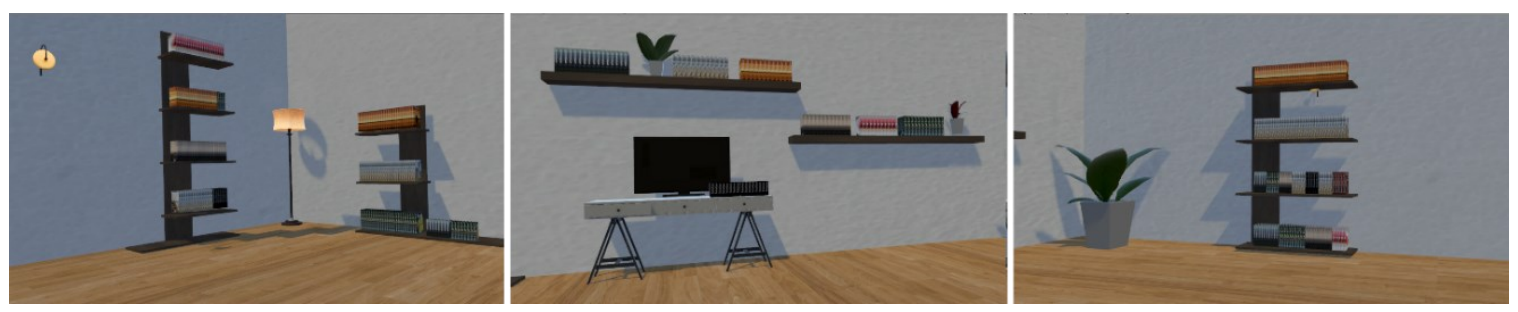

Figure 19: Desktop scene, low-density virtual library with 425 books in it.

Medium density: In order to increase the number of books and make the

environment more cluttered, we first scaled the book models to be narrower to fit more books in the same amount of space on each shelf. We then added more books on half of each row on top of the previous ones. Consequently, we had 1,058 books in the mediumdensity virtual library. See Figure 20.
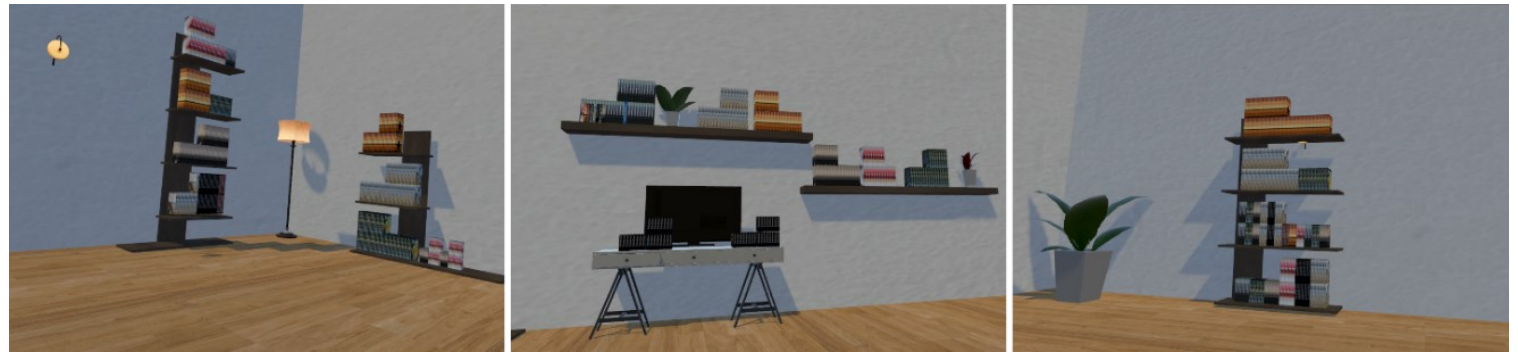

Figure 20: Desktop scene, medium-density virtual library with 1,058 books in it.

High density: We made the width of books even thinner than in the medium density, then we added a full row of new books on top of each previous row for both shelves and the desk to make the environment more crowded. Hence, we had a total of 1,901 books in the high density. See Figure 21.
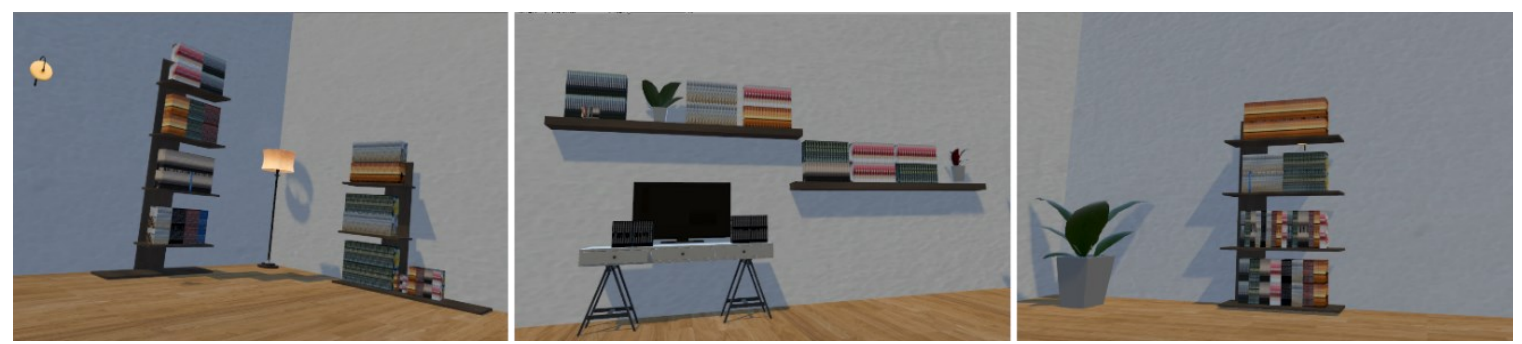

Figure 21: Desktop scene, high-density virtual library with 1,901 books in it. 


\subsubsection{Procedure}

The experiment was conducted remotely due to the COVID-19 pandemic rendering inperson studies impossible. To limit the risk of spreading COVID-19 in our experiment, we asked interested people first to fill out a screening form regarding having any symptoms or being in contact with anyone who has had COVID-19 recently. If the answer to all questions was NO, we dropped off the disinfected VR equipment and PC at the participants' home, following physical distancing protocols. To ensure participant privacy, we destroyed the participants' home addresses and contact information as soon as we got back the equipment.

After receiving the equipment, participants were instructed to first read the provided "setup and experiment instructions" file. To ensure a comparable setup among participants, we prepared a section in the instructions to explain how to configure all of the VR equipment on a table, as seen in Figure 22. The PC and the smartphone were preloaded with all required software before dropping them off to participants.

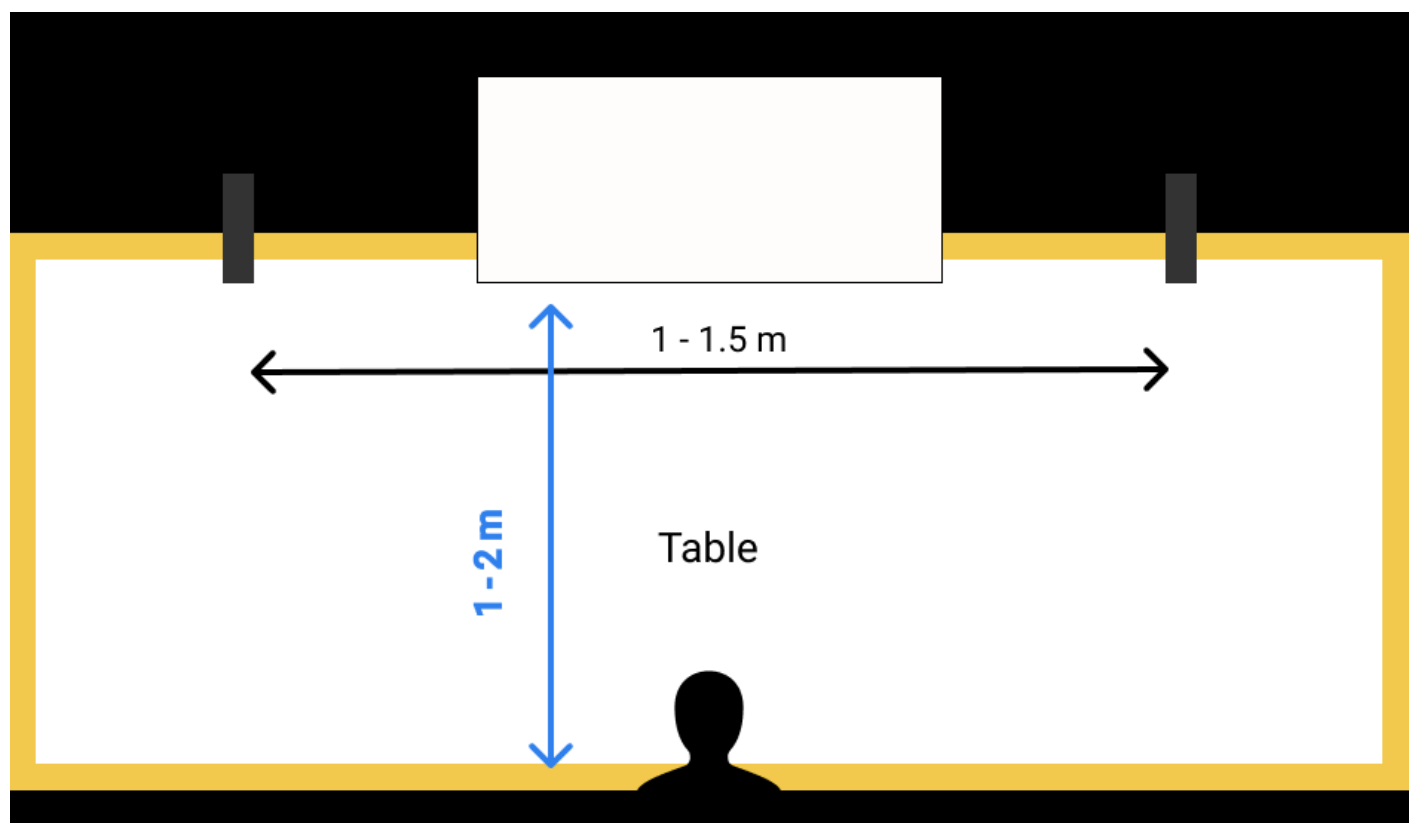

Figure 22: Equipment set up on a table, shown in the "setup and experiment instructions" file. 
Before starting the experiment, participants provided informed consent and completed a demographic questionnaire online through Google Docs as we asked them in the instruction file. Upon starting the experiment, they had to enter the required start-up information (we shared related information with them in an email or in the "setup and experiment instruction" file). See Figure 22. After this, the software started and the experiment began. We put an instruction text inside the VR scene that asked participants to perform the task as fast and as accurately as possible (see Figure 18). For each density with each technique, participants had to find and select the target book from the shelves eight times (i.e., block).

After participants finished all trials with a given technique, they completed a questionnaire about their experience using that technique. After completing the experiment, they also completed a "post-experiment" questionnaire to share their final thoughts and rank the techniques.

Overall, the experiment took about one hour, with participants in VR for $\sim 45-50$ minutes following a $\sim 10-15$-minute setup. Upon completion, they received a $\$ 15$ (CAD) Amazon digital gift card.

\subsubsection{Experiment Design}

Our experiment employed a within-subjects design with two independent variables: selection technique and density. Each had three levels.

Selection technique: MTPR, progressive refinement, multi-touch

Density: low, medium, high 
The selection technique was counterbalanced according to a Latin square. However, we did not counterbalance density, so participants could initially learn how to perform the task at lower (easier) density levels before moving onto high (harder) density.

Our experiment included three dependent variables: search time, selection time, and error rate.

Search time: the time participants spent to find the target book on the shelves in seconds. This time started as soon as participants pressed any key on the keyboard and ended when they opened the selection menu.

Selection time: the time participants spent to select the target book from menus in seconds. This time started as soon as the menu opened and ended when the target book was ultimately selected and the menu was closed. There was no overlap between search time and selection time.

Error rate: the average number of wrong menu selections (as a percent).

Overall, participants completed three selection techniques $\times 3$ densities $\times 8$ targets (one block) for 72 target selections from shelves. Across all 12 participants, this yields 864 target selections from the shelves in total.

\subsubsection{Results}

We employed two-way ANOVA to analyze the significance of selection techniques and densities. Horizontal bars $(\cdots \bullet)$ in bar charts indicate significant pairwise differences with Bonferroni adjustments between different factors.

\subsubsection{Search Time}

The two-way ANOVA revealed that the effect of the selection technique on search time was not statistically significant $\left(F_{2,22}=1.561, p>.05\right)$, yet the effect of density on search 
time was statistically significant $\left(F_{2,22}=122.052, p<.0001\right)$. However, the interaction effect between the selection technique and density was not significant $\left(F_{4,44}=1.808, p>\right.$ $.05)$. See Figure 23.

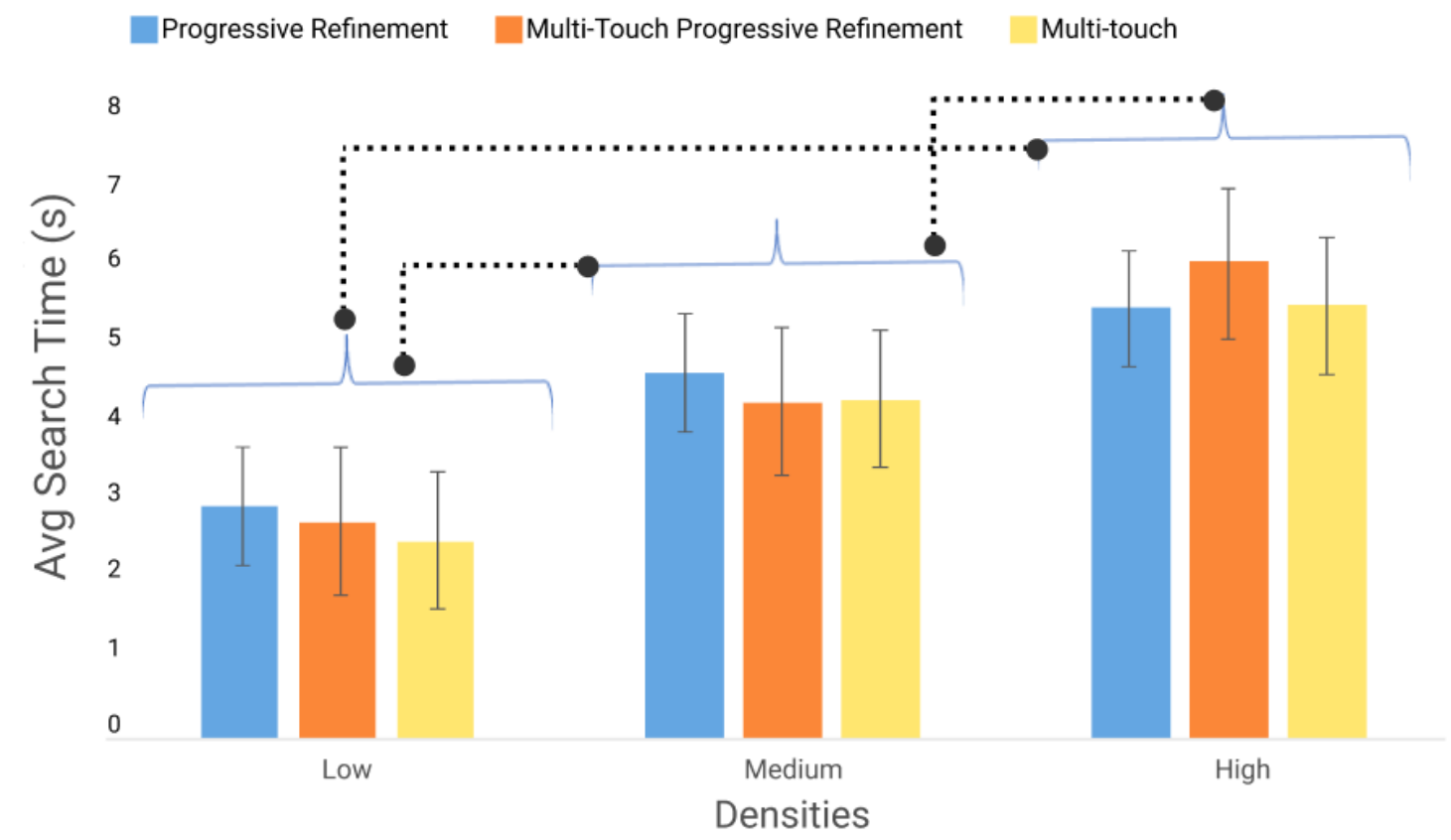

Figure 23: Result of two-way ANOVA and Bonferroni-Dunn analysis for book search time. The error bars represent standard error.

A Bonferroni-Dunn posthoc test $(p<.05)$ on density revealed that regardless of the selection technique, low density required less search time than both medium and high density, while high density was slowest.

\subsubsection{Selection Time}

A two-way ANOVA on selection time revealed that the effect of the selection technique on selection time was statistically significant $\left(F_{2,22}=10.946, p<.001\right)$. It also showed that the effect of density on selection time was statistically significant $\left(F_{2,22}=23.958, p<\right.$ .0001). However, the analysis failed to show any significant interaction effect between selection technique and density $\left(F_{4,44}=0.701, \mathrm{~ns}\right)$. See Figure 24 . 
Then we applied a Bonferroni-Dunn posthoc analysis to determine which technique was efficient in terms of selection time. An analysis demonstrated that, multitouch was faster than progressive refinement and MTPR.

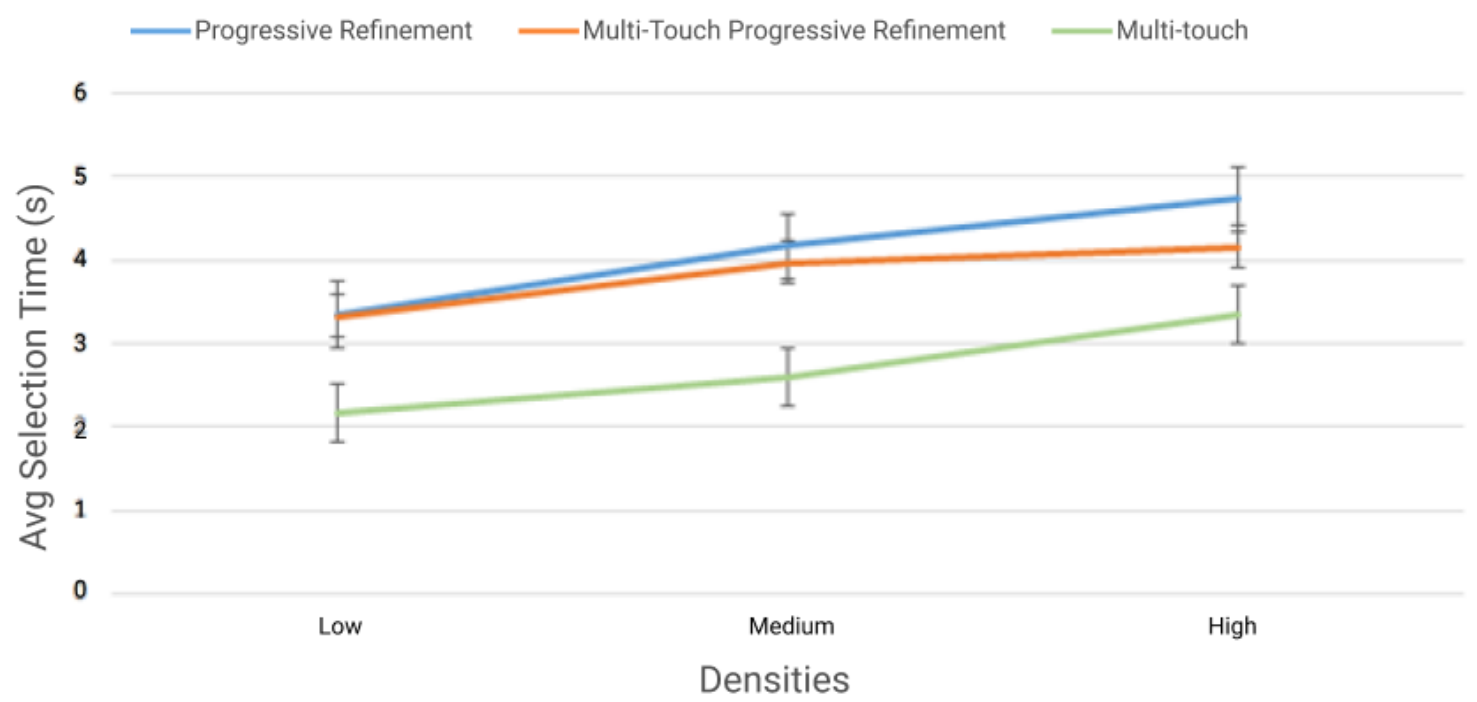

Figure 24: Result of two-way ANOVA and Bonferroni-Dunn analysis for selection time. This is the average selection time for low, medium, and high density per technique. The error bars represent standard error.

\subsubsection{Error Rate}

There are two different types of errors possible in our experiment, one being the navigation error rate and the other the finger error rate. Finger error rate only exists for the MTPR technique and relates to the wrong number of fingers that touch the screen. We analyze these separately below.

\subsection{Navigation Error Rate}

A navigation error occurs when the participant swipes in the wrong direction (in MTPR and multi-touch) or points the ray in a sub-menu (in progressive refinement) in a given menu. When such errors occur, i.e., the participant incorrectly selects a sub-menu, the 
selected sub-menu simply does not open, and the participant has to try again. We do not show any error messages to them to avoid distracting them and increase the search time accordingly. This type of error can occur with all three selection techniques. To calculate the navigation error rate, we counted all menu selections made by a participant in each density. We then divided the number of navigation errors by the total number of selections to get the average number of errors per participant. In the end, we calculated the average number of errors per density which is seen in Figure 25.

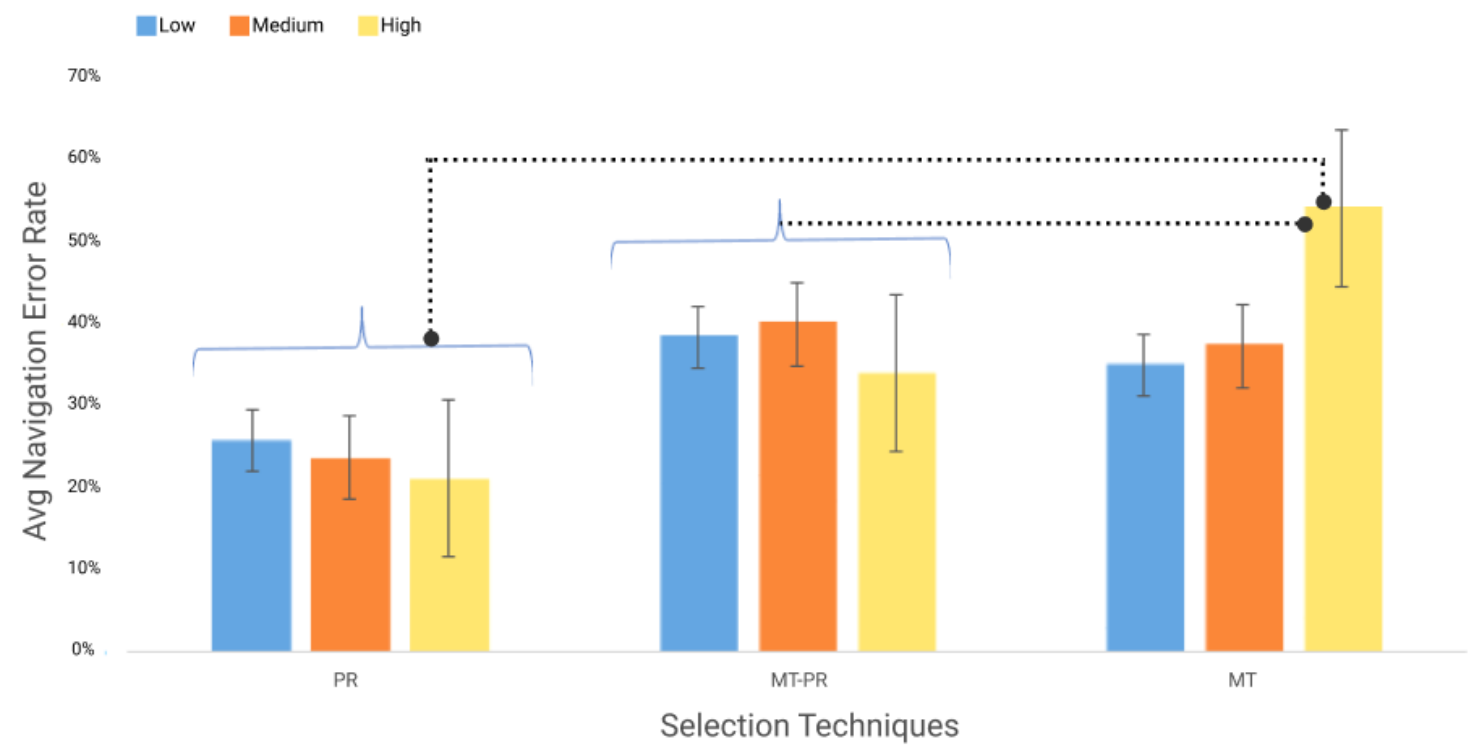

Figure 25: Result of two-way ANOVA and Bonferroni-Dunn analysis for error rate. The error bars represent standard error.

A two-way ANOVA showed that the effect of the selection technique on error rate was statistically significant $\left(F_{2,22}=4.042, p<.05\right)$. Meanwhile, the effect of density on error rate was not statistically significant $\left(F_{2,22}=2.530, p>.05\right)$. The analysis also showed that the selection technique * density interaction effect was not statistically significant $\left(F_{4,44}=0.657, \mathrm{~ns}\right)$. A Bonferroni-Dunn analysis revealed a significant difference between multi-touch and the other two selection techniques. We hypothesized 
that the selection technique influences error rate, and this was confirmed here. However, we had not expected such a high error rate with the multi-touch technique. Some participants mentioned that this could be related to the low touch sensitivity of the smartphone. Of course, the same touchscreen was used for MTPR as well, but since participants see fewer menus with it than multi-touch, the error rate was lower.

We expected that when participants had to put more than two fingers on the smartphone surface, the swiping action gets more challenging with the MTPR technique. Thus, the error rate increases. According to Figure 25, the results showed that it was not as influential as we thought.

\subsection{Finger Error Rate}

A unique characteristic of our MTPR technique is the ability to put multiple fingers on the screen to select a specific item in one of the quad menus. This introduces the possibility that users might put the incorrect number of fingers on the screen. So, in order to select the target object, participants could place the wrong number of fingers three or four times or even more on the screen. We therefore analyzed how often these errors occurred. Since this error is related to MTPR only, we do not compare it with other techniques and report it as a reference for how frequently such errors occurred. See Figure 26 - the number on the graph shows the average number of finger error rate per density.

A one-way ANOVA revealed that the effect of density on this finger error was not statistically significant $\left(F_{2,14}=0.928, \mathrm{~ns}\right)$. This supports our hypothesis regarding finger error rate. This is further supported by some participants mentioning this confusion in the feedback after the experiment. 


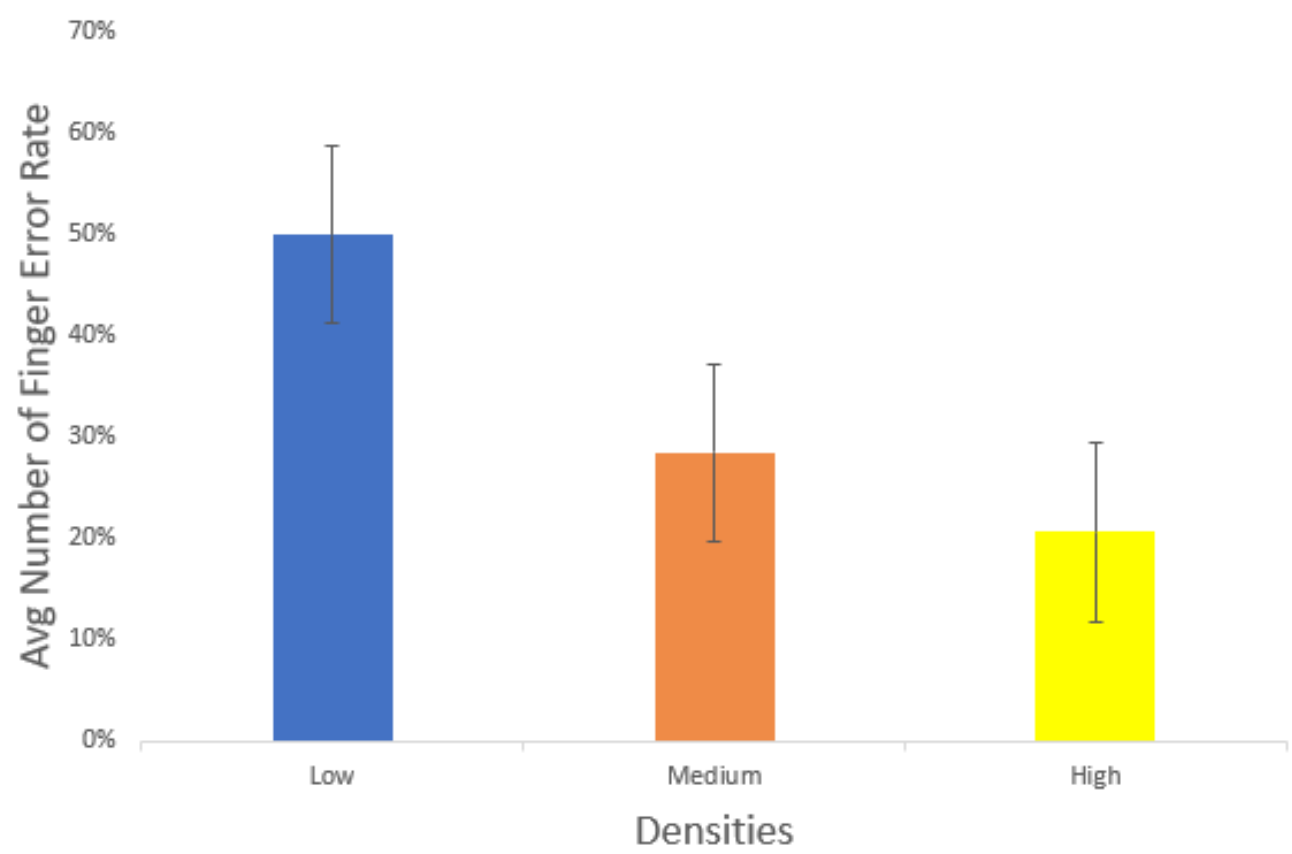

Figure 26: Result of one-way ANOVA analysis for finger error rate.

\subsubsection{Questionnaire Results}

We designed a questionnaire that participants completed after performing each technique. We asked how easy, accurate, and fast each selection technique was and allowed participants to rank the selection techniques, providing their reasons. Figure 27 shows the results of the questionnaires. According to the participants, the most preferred technique was first multi-touch (50\%), then MTPR (38\%), and finally progressive refinement (12\%) last. Participants indicated that they most preferred multi-touch because it was easy and fast to select objects. Many participants mentioned that they were familiar with such actions from smartphone usage. Conversely, MTPR was ambiguous for them, and progressive refinement was harder than the other two techniques to perform.

Participants also mentioned that the concept of MTPR made sense, as it reduced the number of menus, but they found it difficult to determine how many fingers to use to select the desired object. 


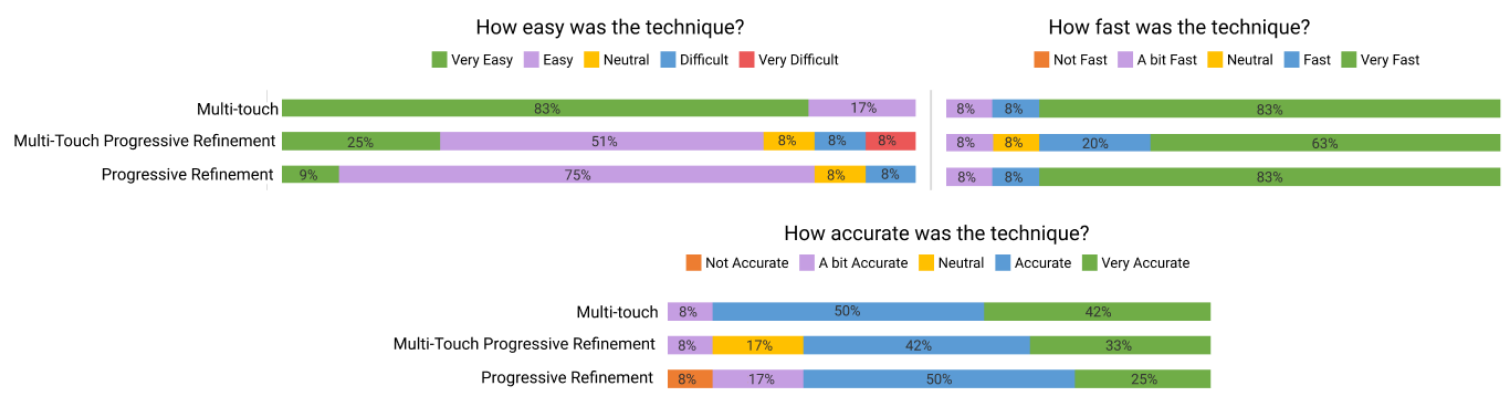

Figure 27: Selection technique usability and assessment questionnaire. Label numbers indicate the percentage of participants choosing each answer.

\subsection{Discussion}

Overall, our analysis found that the selection technique did not influence search time, but density did. When the density increased, search time increased accordingly. This makes sense since visual search is known to increase linearly with the number of items being searched [4].

We hypothesized that MTPR offers lower selection time relative to standard progressive refinement. However, according to our results, while selection time is better, the difference is not significant. We anticipate that even more densely packed environments would further separate these two techniques, i.e., that MTPR will perform much faster than progressive refinement. One reason for that is users do not need to dig deeper in menus and can select the target object sooner. However, our results also indicate that multi-touch is faster than MTPR, likely because of its simplicity and familiarity. Users neither need to think about the number of fingers they have to put in the screen like MTPR, nor need to move their hand to point the ray on the target menu like progressive refinement.

Another interesting finding was that progressive refinement was the most accurate technique. In contrast, the number of navigation errors was high with multi-touch, 
especially in high density. This could be due to a lack of smartphone touchscreen sensitivity, which was mentioned by some participants in their questionnaires. This problem could happen for MTPR as well, but since menu navigation is reduced with this technique, the navigation error rate decreased. Overall, while multi-touch was the fastest, and progressive refinement was most accurate, MTPR was consistently between them in terms of both speed and accuracy (Figure 24 and Figure 25). This suggests our technique has promise for situations calling for a compromise between speed and accuracy.

Finally, we did not compare finger error in techniques since it only exists for the MTPR. As we hypothesized, this error rate is high and likely the main reason the technique yielded a higher selection time in the MTPR. We decided this was a key aspect to improve prior to running another user study.

\subsection{User Study Limitations}

The primary limitation of our study was lack of tutorial/practice session for users. This point affected the experiment negatively, since we believe that users were learning about techniques during their performance in the low density and thus got better in the medium and high density.

The second limitation is that we did not run any pilot study before our experiment. We could have found out some of our technique weaknesses during a pilot study and fixed them before running the first user study.

Lack of density counterbalancing is another problem that may also have had some influence on the data and user experience.

Also, we were limited in the number of participants we could recruit due to the COVID-19 pandemic. Although we provided the setup and experiment instructions to 
participants, there were participants who got confused about understanding and performing some techniques intensely, such as MTPR. Also, due to some time spent setting up the equipment, we limited the number of trials and blocks to make the experiment shorter than an hour for participants.

\subsection{Conclusion}

This experiment revealed that multi-touch was faster than both techniques, but less accurate, especially in high densities. This result was opposing to our hypothesis, and we believe this result can be traced to inability to determine how many fingers should be used to select a given item with MTPR. The next chapter describes the improvement we applied on MTPR and the second experiment for which we improved this issue. 


\section{Chapter 4: Design and Implementation of IMPReSS Technique - Improved Multi-Touch Progressive Refinement Selection Strategy}

Results of the first study revealed that participants were confused about the number of fingers they had to use to select the desired object in a given menu when using our MTPR technique. As we report in section 3.3.6.4, some participants mentioned this explicitly in their questionnaires. Hence, we decided to improve our technique to make it easier for participants to determine how many fingers mapped to a given object position in each menu. We also devised an algorithm to better order objects distributed inside the quad menus not to be randomly positioned. We dubbed the improved version of the technique Improved Multi-Touch Progressive Refinement Selection Strategy or IMPReSS for short. All steps were the same as the previous technique except for step 2: showing selected objects to users. The improved version is explained below.

\subsection{Distributing Selected Objects Inside the Quad Menu}

To implement this enhancement, we introduced some predefined invisible spaces within each quad menu. Then we dedicated a specific quad menu number and position number to them and made a list. For example, inside quad menu number 1 , we created a spatial position, with $\mathrm{x}=0.5, \mathrm{y}=0.2, \mathrm{z}=0.2$ (three-dimensional coordinate system) and saved it as first position in the system. Then we put some space (i.e., space_ $\mathrm{x}=1$ for objects in one row) and created the second spatial position — the space between objects varied based on the number of objects inside the menu.

Thus, we defined 64 spatial positions, a maximum of 16 inside each quad menu to cover all menu levels. See Figure 28. After selecting an initial group of objects via 
sphere-casting, we mapped the position of the objects into those predefined spatial positions and distributed them inside each menu based on the new order.

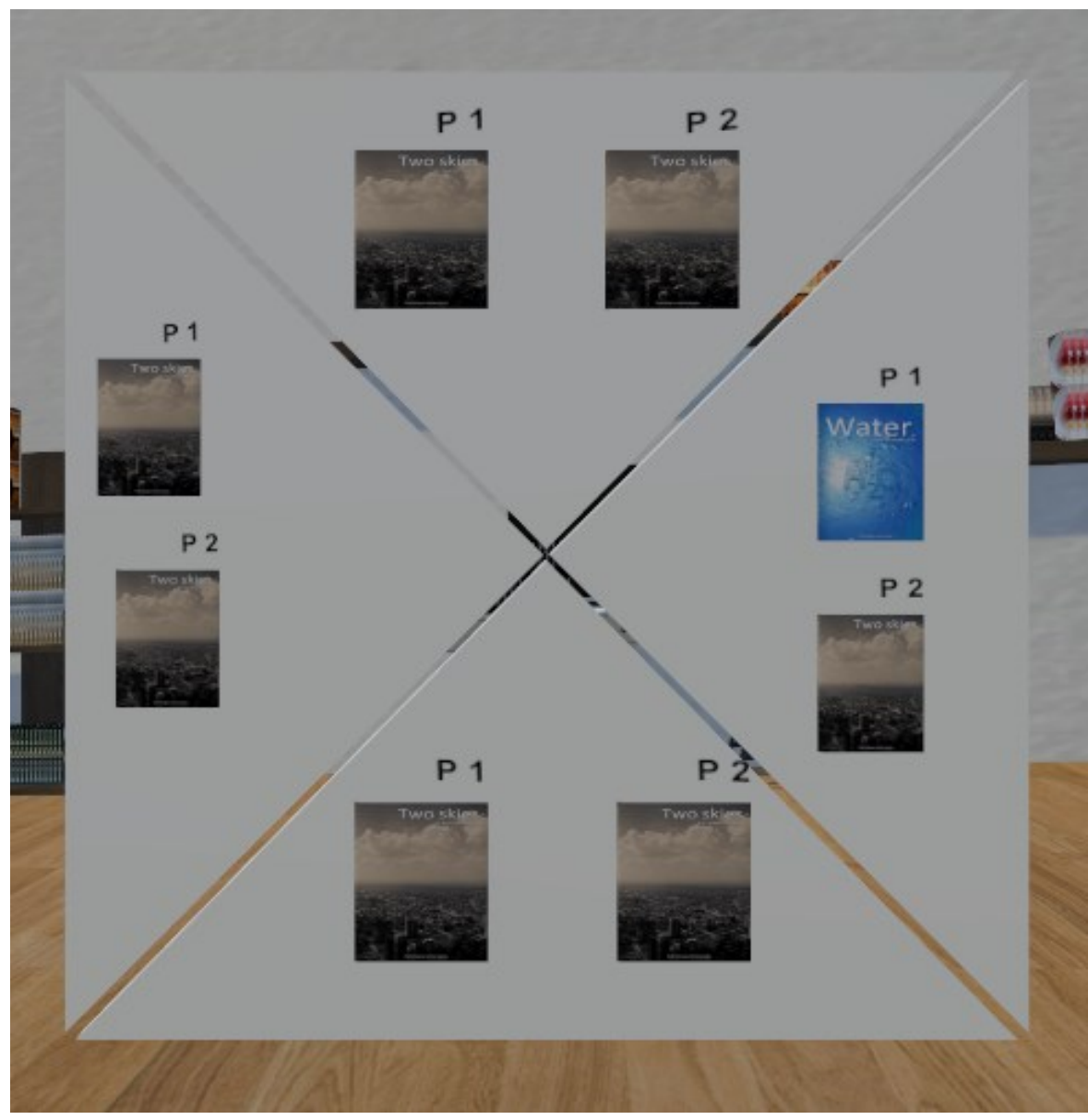

Figure 28: IMPReSS technique quad menu, depicting book positions as text (i.e., number of fingers to select a given item in the menu). For example, the Water book is assigned to position 1; touching one finger to the screen and swiping right would select it.

To ensure that objects were distributed equally inside each menu quadrant (i.e., no menu without any objects or menus full of too many objects), we dedicated one object at a time to each menu. We first filled all the first positions inside the four quad menus, then filled the second positions of them, and so on. For example, when six objects were initially selected by sphere-casting (Figure 29, right), we distributed them as follows inside quad menus: 
I. Yellow book into menu number 1 in the first position

II. Purple book into menu number 2 in the first position

III. Red book into menu number 3 in the first position

IV. Grey book into menu number 4 in the first position

V. Green book into menu number 1 in the second position

VI. Blue book into menu number 2 in the second position

Finally, we also displayed each object's position number inside all menus as text to participants (see Figure 28). We decided to show "P" (not the word position) plus position number only as a guidance label to prevent making any extra clutter inside the menu.
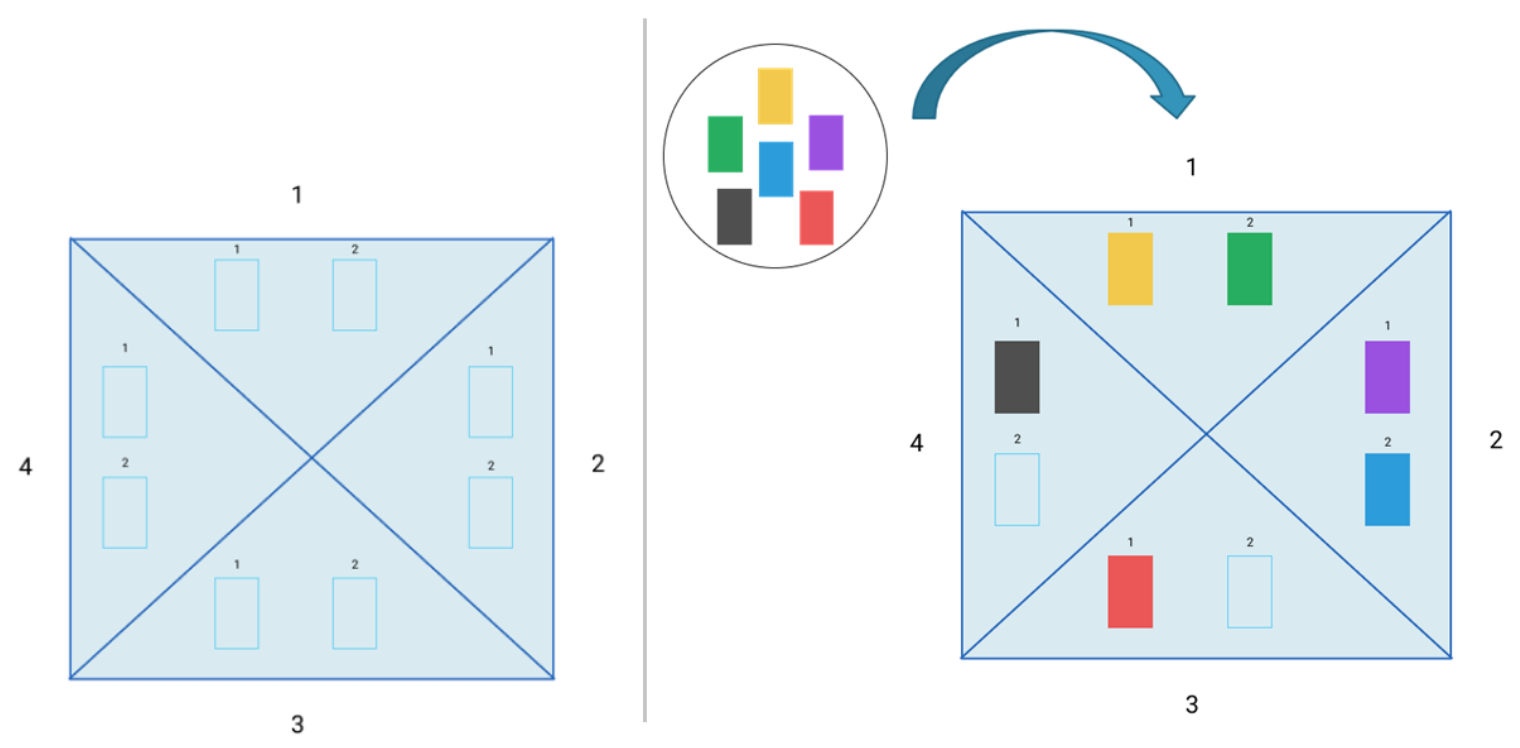

Figure 29: Ordering objects inside the quad menu according to IMPReSS technique. Left) creating some predefined spatial positions and dedicated specific menus and position numbers. Right) distributing selected objects according to IMPReSS ordering.

We designed this to help participants select the desired object as soon as the quad menu opens, therefore improving selection time. 
With IMPReSS, when there are more than four items in a menu, we do not display the object position numbers; these are only shown when there are four or fewer objects inside each quad menu.

\subsection{MTPR and IMPReSS Technique Limitations}

A limitation of both MTPR and IMPReSS is that these techniques do not work well with small touch screens (any touch screens less than four inches), since one finger placement on the touch screen may block the whole screen. For instance, it would be very hard or even close to impossible to apply this technique on smartwatches (which usually have 1.2 to 1.7 inch touch screens).

Besides, the current handheld smartphone implementation of MTPR and IMPReSS requires both hands to perform the interaction: one hand to hold the phone and the other to place fingers on the screen. In the future, we can work on implementing this technique to use only one hand. Scalability will be an essential factor in that case which we need to consider. We can fix the smartphone position by putting that on a handle and fix it to a table and make one of the user's hands free.

Since the technique is relying on touching and swiping on the screen, touch screen sensitivity is an important factor, and users may have a different experience if they have different touch screens. Results also could be affected by changing the touch screen. For example, if the touch screen sensitivity is high, detecting number of fingers and swipe gesture is recognized faster by the device and it decreases selection time. This factor improves user experience and affect their ranking in the post-experiment questionnaire as well. 
Another limitation here is that it could be hard to interact and select objects in this technique while users are in positions like walking or running. This is because while people are walking or running, it is harder to concentrate on the screen to find and select objects. It would also be harder to select any object by putting four fingers on the screen while they are running.

Finally, people who have hand/finger motor disabilities might not be able to use this technique since the number of fingers touching the screen matters in this technique.

\subsection{User Study 2: Evaluating the IMPReSS Technique}

We conducted a user study to evaluate IMPReSS, our improved version of MTPR.

Similar to the first user study, we compared our technique to progressive refinement and multi-touch in terms of search time, selection time, and selection accuracy in this study. Our hypothesis was that IMPReSS has lower selection time and lower error rate than progressive refinement and multi-touch in all densities. We also hypothesized that finger error rate in IMPReSS is lower than finger error rate in MTPR at experiments' same densities only.

\subsubsection{Participants}

We recruited 12 participants ( 4 men, 8 women) for this study. Ten of these had also participated in the first study. Since the second study was performed during the COVID19 pandemic, we recruited all participants remotely. Hence, we had to drop off all essential equipment at participants' places.

Participants were between 26 and 42 years old $(\mu=32.75, S D=4.2)$. We asked them to share their level of experience with VR systems in the questionnaire: $33.3 \%$ had some VR experience before (used VR 4-5 times overall), while 66.7\% had no VR 
experience. Also, none of participants were professional in VR (use VR at least three times per week). All were right-handed. All participants had a normal or corrected-tonormal stereo vision, assessed based on questioning during recruitment.

\subsubsection{Apparatus}

The equipment used in this study was similar to that in the first user study. Like the first user study, to avoid spreading COVID-19, we dropped off all main hardware (see Figure 30) at the participants' residence, applying social distancing and privacy protocols.

Hardware that would not influence the experiment (e.g., monitor, keyboard, and mouse) were not included as part of the dropped-off equipment; participants used their own devices.

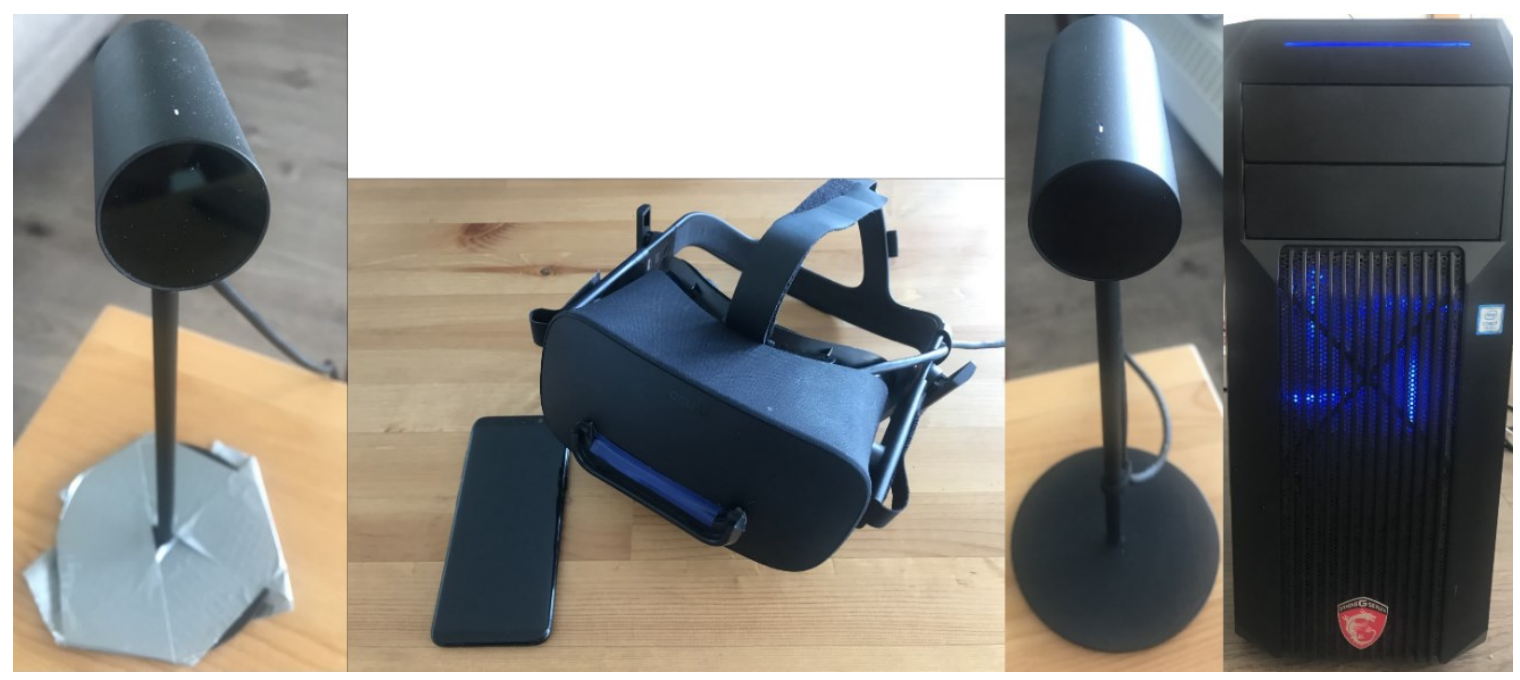

Figure 30: Essential equipment used in the experiment. From left, the first image is related to sensor $360^{\circ}$ tracking, the second image is the Oculus Rift with the Samsung Galaxy S8, the third image is another sensor $360^{\circ}$ tracking, and the fourth image is the $\mathrm{PC}$ we used.

\subsubsection{Hardware}

In this experiment, we used a PC with an Intel Core i7-7700K CPU at 4.20GHz with 32 GB of RAM, with an Oculus Rift CV1 head-mounted display and two-sensor $360^{\circ}$ 
tracking devices. We performed the experiment using a Samsung Galaxy S8 (OS Android 9.0) as a VR controller (Figure 30).

\subsubsection{Software}

We used the same software as in the first study (Unit3D version 2019.2.10 on Microsoft 64-bit Windows 10, Unity Android 7.0) for developing our VR scene on both desktop and smartphone. The software presented a virtual library scene, with the objective being to find a specific book (Figure 31) and selecting it as fast and as accurately as possible.

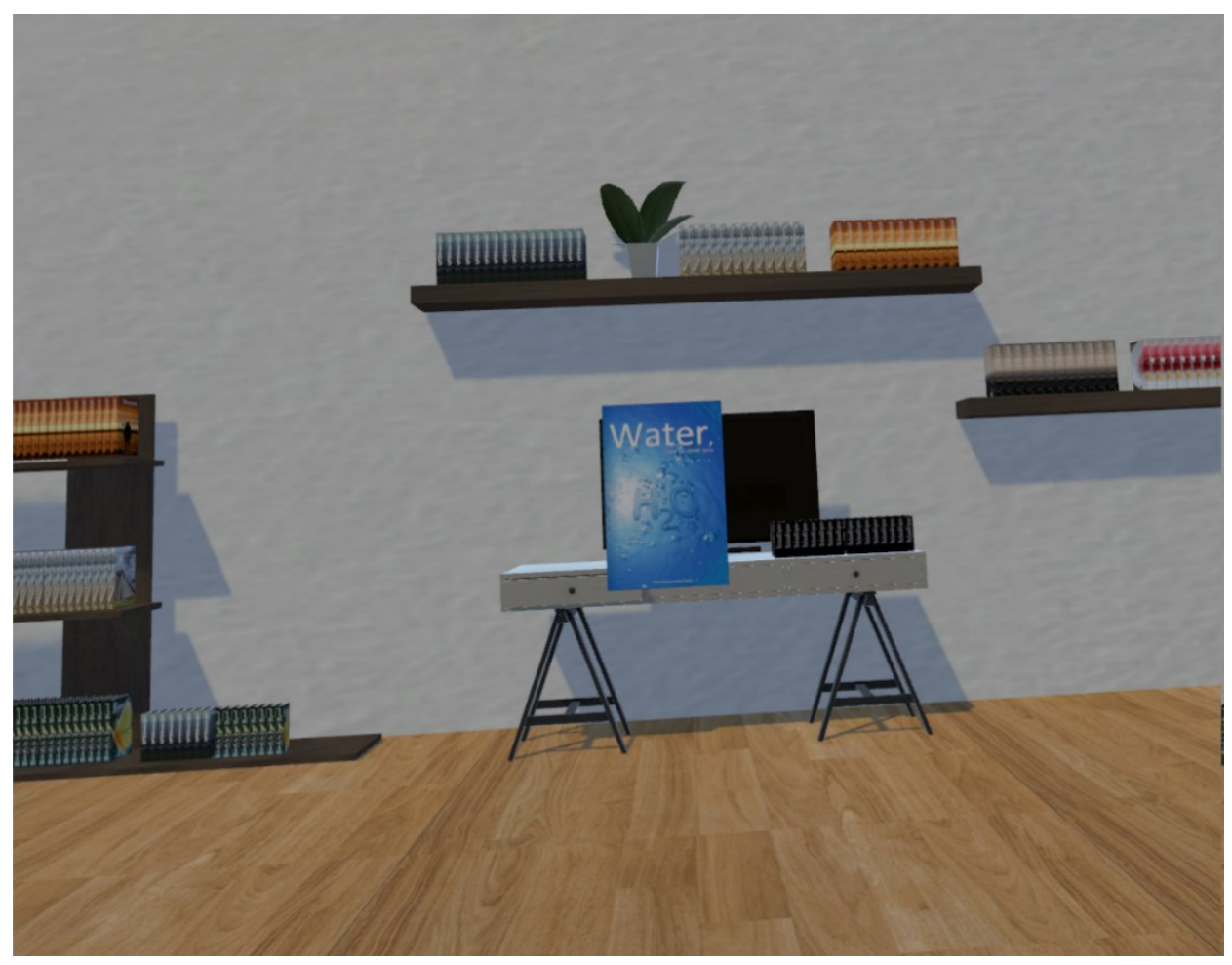

Figure 31: The blue book is the target to find inside the library's shelves.

Participant ID (PID), number of blocks, interaction technique, and density were obtained by the software (see Figure 32). Search time, selection time, and error rate were calculated while the software was running and stored in an Excel file as an output to be used for analysis. 


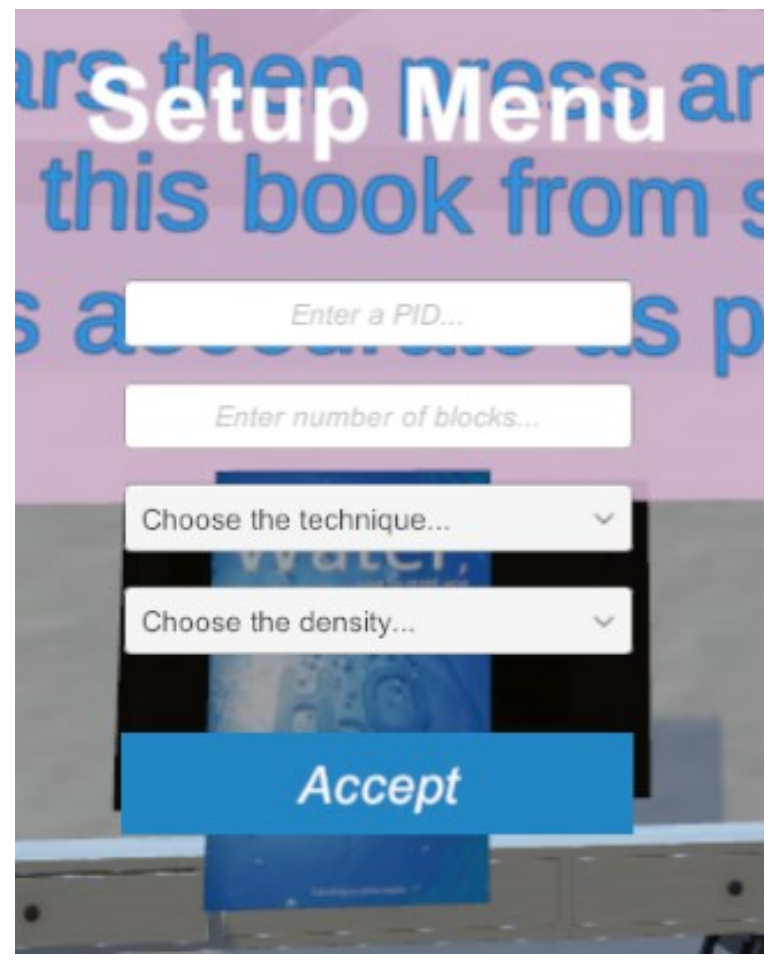

Figure 32: Setup menu shown to participants upon starting the program to enter participant's ID, number of blocks, selection technique, and density.

To further study the effect of environment density, we omitted the original lowdensity condition and added a new high-density condition for this experiment. Thus, new densities could be described as follows:

Low density: We set the previous medium-density as the new low density for this experiment. Consequently, we had 1,058 books in the new low density (see Figure 33).
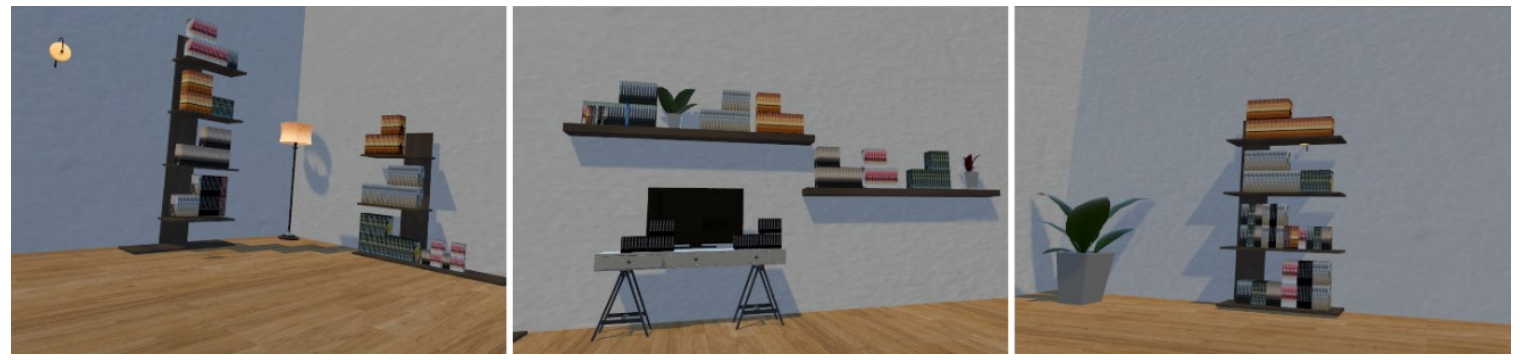

Figure 33: Desktop scene, low density of the virtual library with 1,058 books in it. 
Medium density: The previous experiment's high density condition replaced the medium density in this experiment. Hence, we had 1,901 books in the new medium density (see Figure 34).
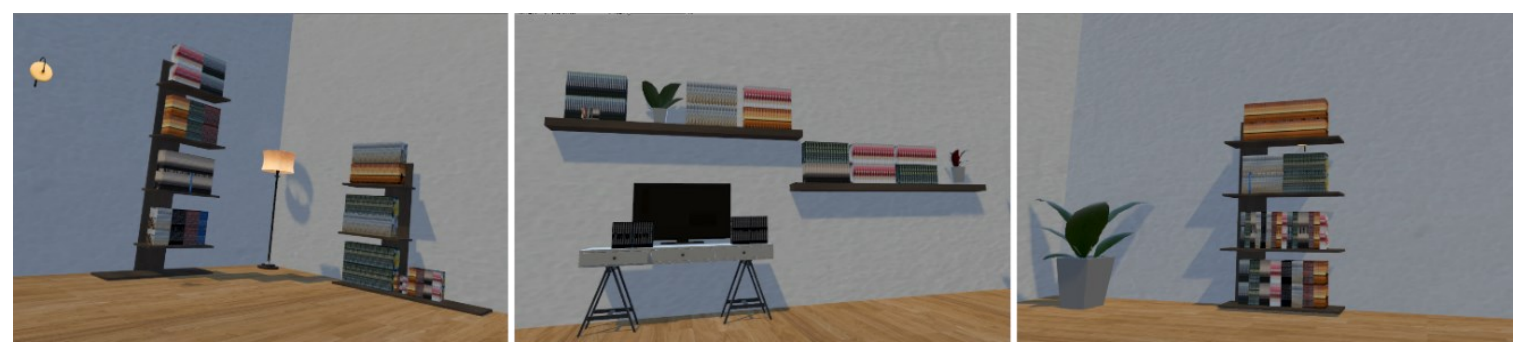

Figure 34: Desktop scene, medium density of the virtual library with 1,901 books in it.

High density: We created a new "super" high density condition, with more books than the previous experiment's high density. This used the same five shelves we had previously. To make the environment more densely packed with virtual objects, we decreased the books' width, making them thinner so we could place more books on each bookshelf. Then, we added three rows of books on shelves with one level and two rows of books on shelves with more than one level. As a result, we had 3,055 books in the scene for the new (super) high density. See Figure 35.
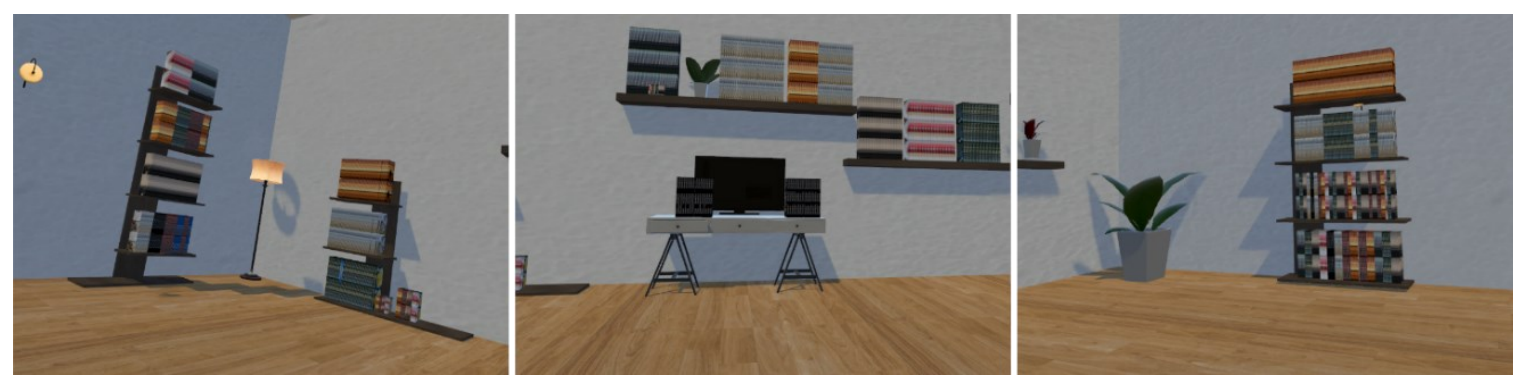

Figure 35: Desktop scene, high density of the virtual library with 3,055 books in it.

Selection techniques were counterbalanced according to PID. We shared related PID with participants by email, which assigned them to a group to counterbalance condition ordering. All participants completed each density, starting with low and ending with high density for each selection technique. We did not counterbalanced density to 
prevent any ambiguity for users. We wanted them to get familiar with the technique first with low density which was easier to interact with. To start the trial, which placed the target book on a library shelf pseudo-randomly to select, participants had to press any key on the keyboard. The target book was positioned in different pseudo-random positions inside each shelf. These positions were different from the first study. Upon pressing a key after the last selection trial, the experiment ended.

\subsubsection{Procedure}

We conducted all experiment sessions remotely due to the COVID-19 pandemic. All applicants who were interested in participating in the study first had to complete a COVID-19 screening form regarding having any symptoms or being it contact with anyone who has had COVID-19 recently. If their answer to all the questions was NO, we considered them participants and dropped off all essential hardware for each of them at their places. We emailed a PDF to participants that included instructions on setting up and performing the experiment in addition to setting virtual meetings via Zoom or Skype to assist them.

After receiving the experiment equipment, participants were instructed to first read the setup and experiment instructions to know how to perform the study. To ensure consistent equipment setups, we included table setup instructions (see Figure 36) in the instruction file. The experiment software was pre-loaded on the provided PC and smartphone. We sent all instructions and Google forms to participants via email. 


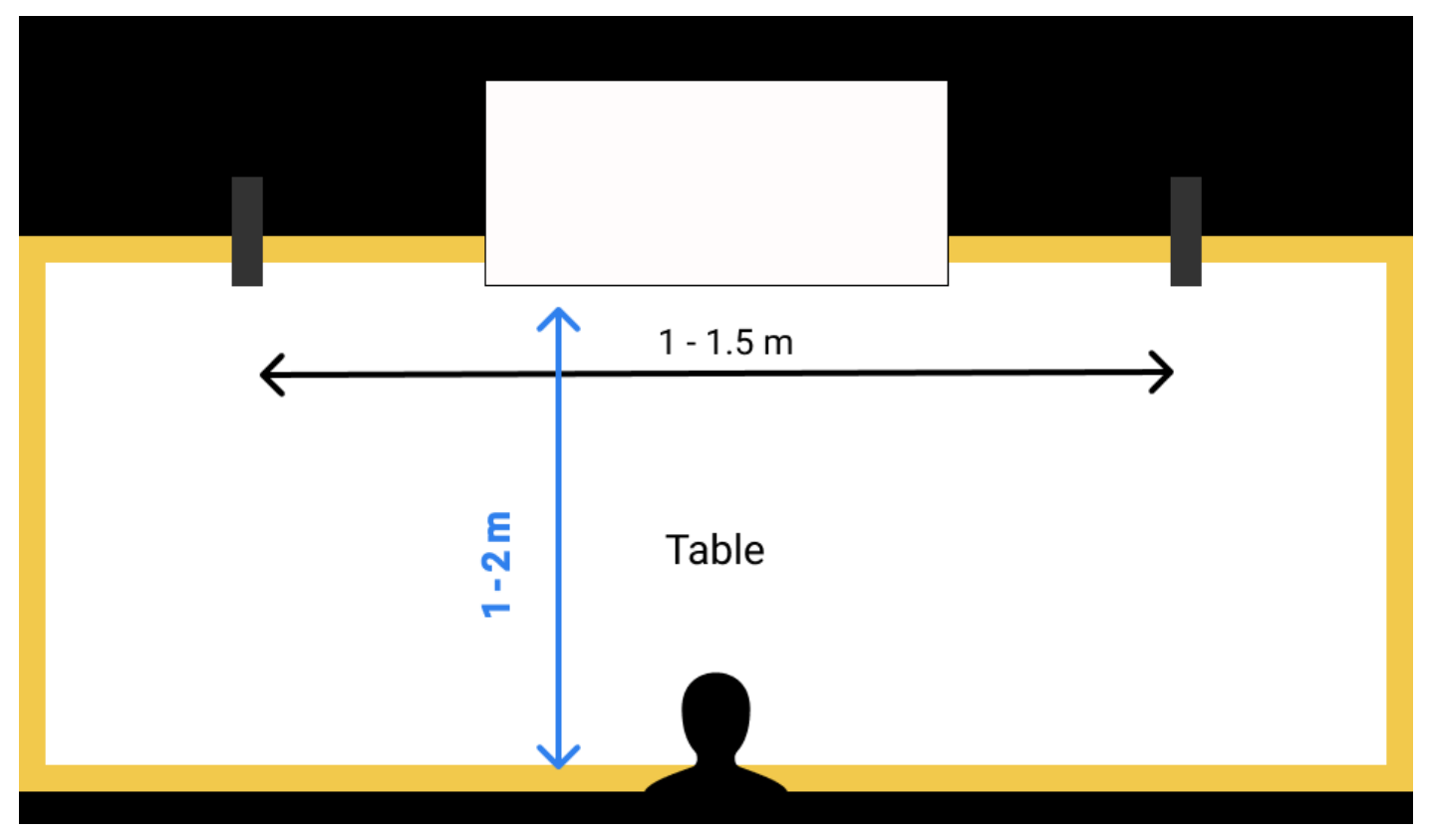

Figure 36: Equipment set up on a table, shown in the "setup and experiment instructions" file. After setting up the equipment, participants read and signed the provided consent form. They then completed a demographic questionnaire. After this step, they were ready to start the VR experiment. Each participant received a specific PID via instruction emails. After starting the software on the PC, they had to fill in setup information (see Figure 32) and enter their dedicated PID, the number of blocks, the interaction technique (their order was different according to the PID), and the density. Upon pressing the accept button, the program started, and the instruction text was presented (see Figure 37). Pressing any key on the keyboard placed the target book in a random position. Upon pressing any key after the last trial of each density (end of the block), the system removed all books from the library shelves, which indicated to participants that the block was over. 


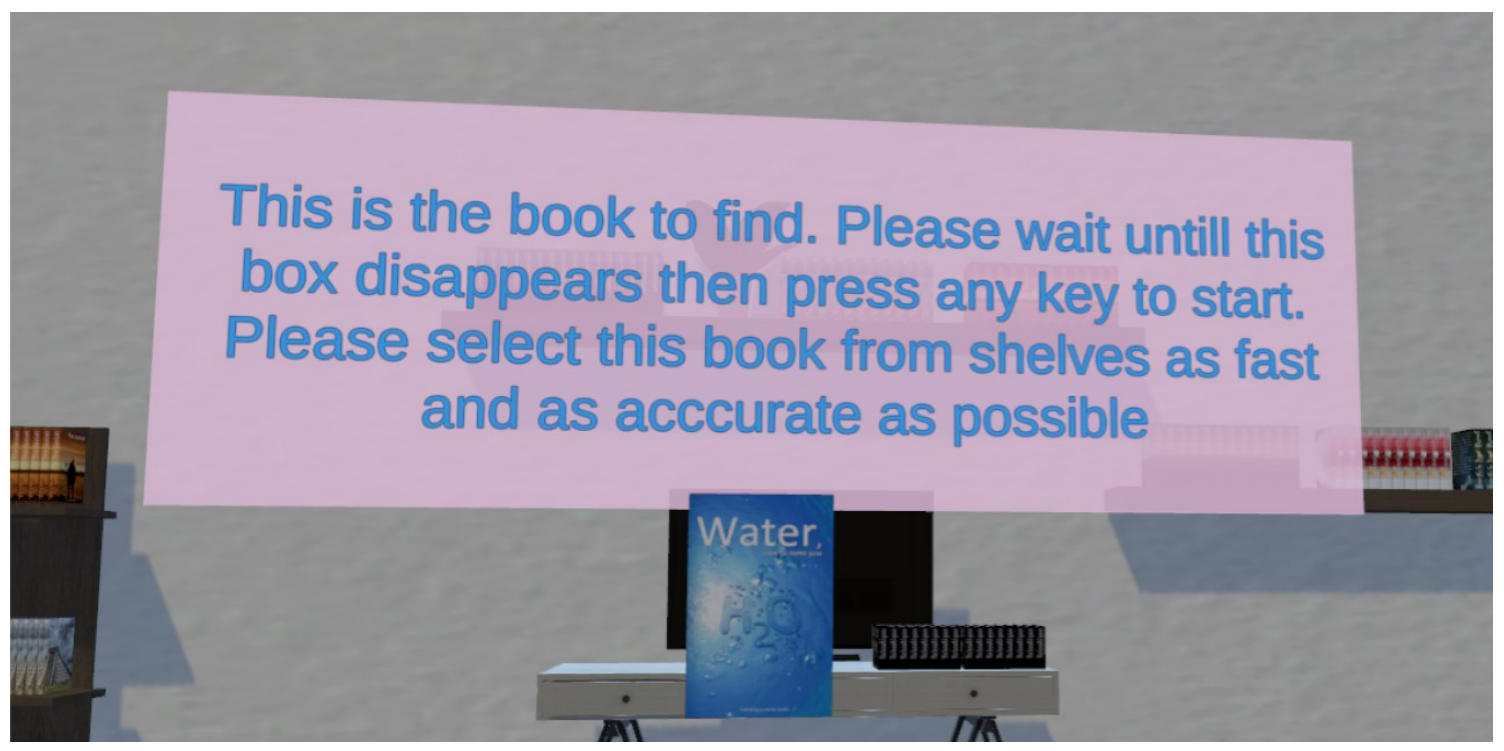

Figure 37: The scene instruction text regarding performing the experiment as fast and as accurately as possible.

After completing all density levels for a given selection technique, participants completed a "device assessment and usability" questionnaire related to the technique and share their experience using it. Participants could take breaks as needed between each block. At the end of the whole experiment (completing all three selection techniques), they completed a "post-experiment" questionnaire and ranked the selection techniques. Overall, the experiment took $\sim 45-50$ minutes for the VR part, following $\sim 10-15$ minutes for setting up the equipment. Participants received a \$15 (CAD) digital Amazon gift card as compensation for participating in the experiment.

\subsubsection{Experiment Design}

We employed a within-subject design with two independent variables, selection technique and density, with each of them having three levels:

Selection technique: IMPReSS, progressive refinement, multi-touch

Density: Low, medium, high 
We also had three dependent variables in this study: search time, selection time, and error rate. These are the same dependent variables as in the first user study, as described earlier in section 4.4.

In total, participants completed three selection techniques $\times 3$ densities $\times 8$ targets (one trial) for 72 selections from shelves. 12 participants $\times 72=864$ target selections from shelves in total for the whole study.

\subsubsection{Results}

Since we had two independent variables, selection technique and density, we employed two-way ANOVA. Horizontal bars $(\cdots \cdot)$ in bar charts indicate significant pairwise differences with posthoc adjustments between different factors.

\subsubsection{Search Time}

Two-way ANOVA showed that the effect of the selection technique on search time was not statistically significant $\left(F_{2,22}=2.161, p>.05\right)$. It also revealed that the effect of density on search time was statistically significant $\left(F_{2,22}=911.721, p<.0001\right)$. The interaction effect between selection technique and density was also not significant $\left(F_{4,44}=0.324, \mathrm{~ns}\right)$

To determine a pairwise difference of density, we applied a Bonferroni-Dunn posthoc analysis. This revealed that lower densities required less search time than higher ones, as expected. See Figure 38. 


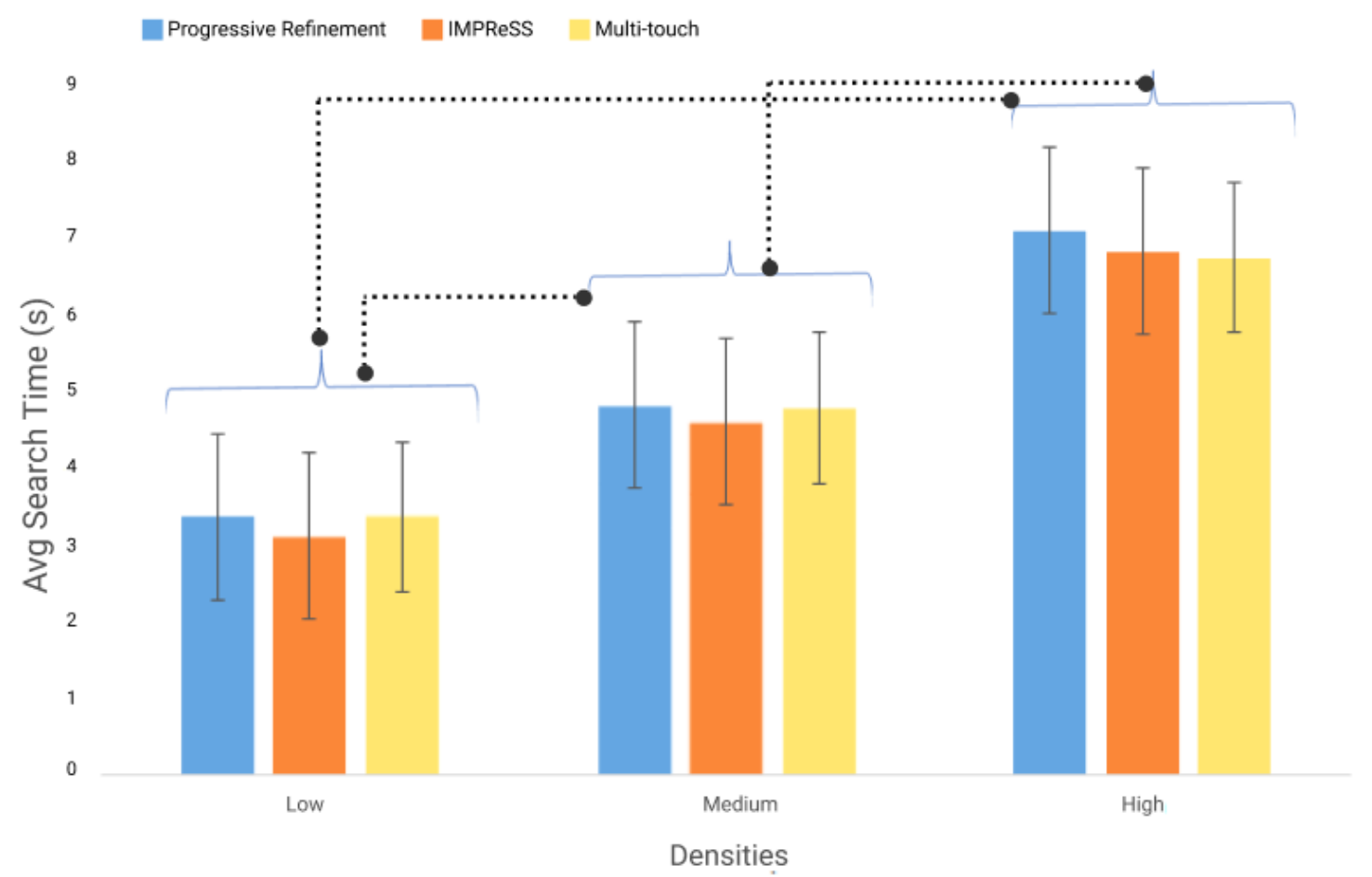

Figure 38: Result of two-way ANOVA and Bonferroni-Dunn analysis for book search time. The error bars represent standard error.

\subsubsection{Selection Time}

We applied two-way ANOVA on selection time, which revealed that the effect of the selection technique on selection time was statistically significant $\left(F_{2,22}=85.764, p<\right.$ .0001). Similarly, the effect of density on selection time was statistically significant $\left(F_{2,22}=25.249, p<.0001\right)$. Interestingly, the analysis revealed that the selection technique and density effect was statistically significant $\left(F_{4,44}=4.403, p<.005\right)$. As seen in Figure 39, IMPReSS offered a much faster selection than the other two techniques. 


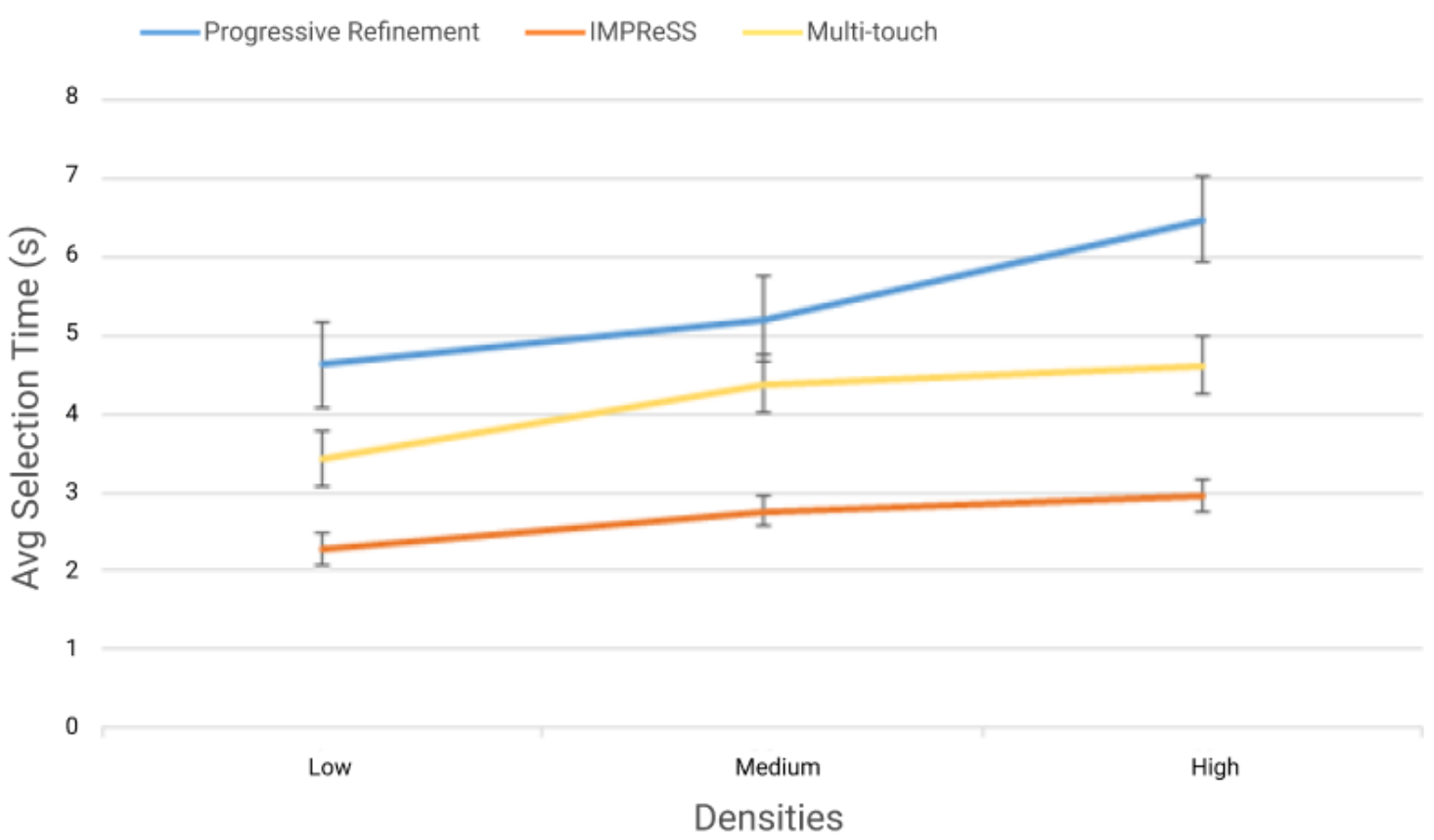

Figure 39: Result of two-way ANOVA analysis for selection time. The error bars represent standard error.

A Bonferroni-Dunn posthoc test revealed that IMPReSS was significantly faster than the other two techniques regardless of density. This finding supports our hypothesis that distributing books inside the menu in a specific order and providing position labels for objects on the menus helped participants select their intended object faster. As seen in Figure 39, the multi-touch technique also performed better than progressive refinement, especially in high density.

\subsubsection{Error Rate}

Like in the first study, we have two different error rates in this experiment. The first is the navigation error rate with all selection techniques and the second is the finger error rate dedicated to the IMPReSS. 


\subsection{Navigation Error Rate}

A navigation error occurs when swiping in the wrong direction (in the IMPReSS and multi-touch technique) or by selecting the wrong menu using the ray (in progressive refinement) inside each menu. Note that if participants select a wrong sub-menu, it did not actually open in our experiment and participants just had to try the selection again. We designed it in this way to reduce variability in selection times due to navigating into incorrect menus.

The navigation error rate was calculated as the percentage of erroneous menu navigations out of all menu selections for a given participant and experiment condition. We then calculated the average number of errors per density. Two-way ANOVA revealed that the effect of the selection technique on error rate was statistically significant $\left(F_{2,22}=\right.$ $11.708, p<.0005)$. However, the effect of density on error rate was not statistically significant $\left(F_{2,22}=3.139, p>.05\right)$, nor was the interaction effect between selection technique and density $\left(F_{4,44}=0.948, n s\right)$. Surprisingly, progressive refinement's error rate was higher than two other techniques in this experiment. See Figure 40. IMPReSS had the lowest error rate compared to other techniques, which means it was the most accurate technique in the second study. It is also lower than the version used in the previous study, which suggests the improvements to the technique also enhanced accuracy. 


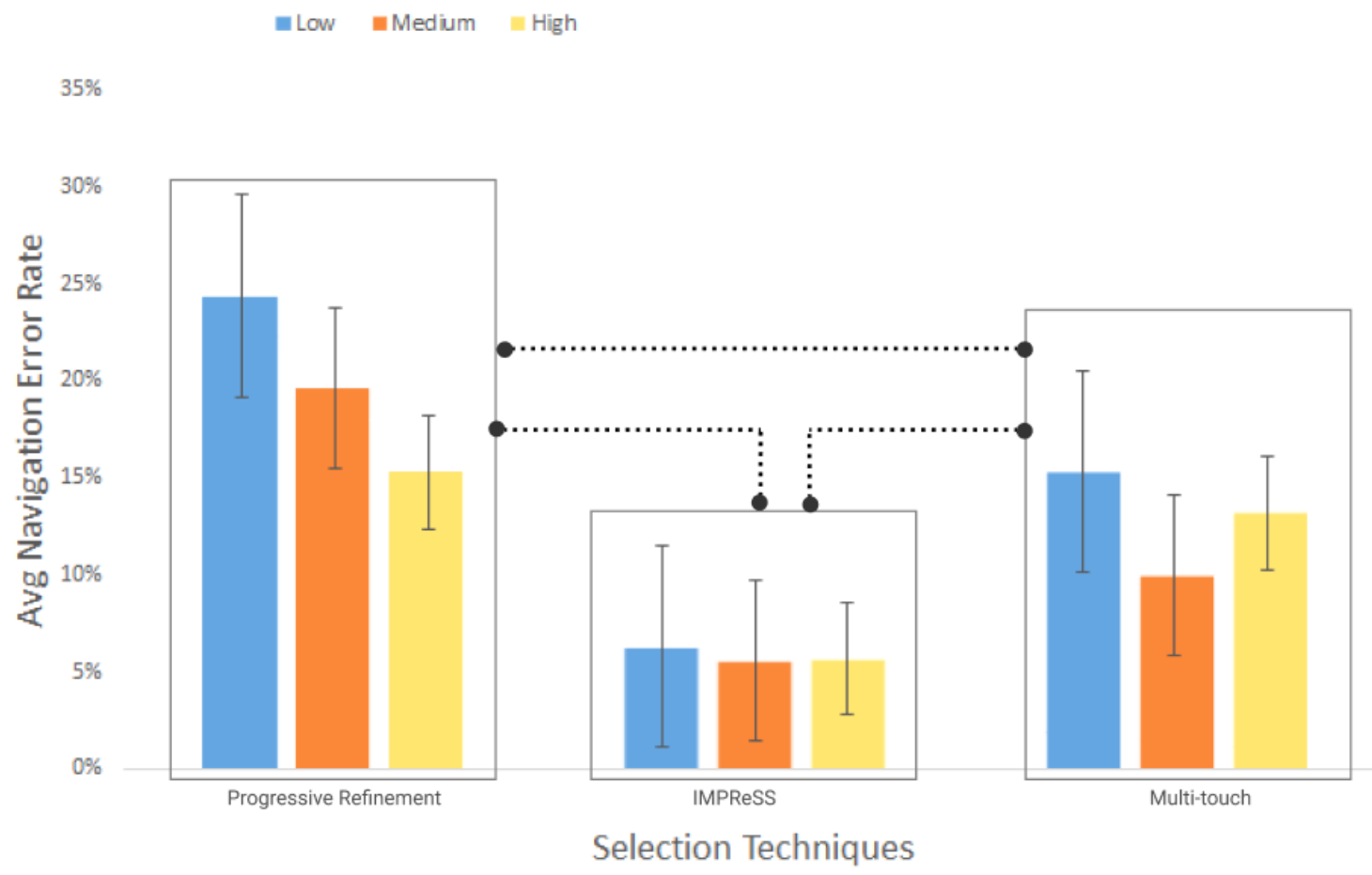

Figure 40: Result of two-way ANOVA and Bonferroni-Dunn analysis for the navigation error rate.

The error bars represent standard error.

\subsection{Finger Error Rate}

The finger error only exists for the IMPReSS selection technique. It is defined as having the wrong number of fingers on the screen when selecting an item (i.e., selecting the wrong item in a given sub-menu due to placing the wrong number of fingers on the screen during a swipe). Hence, we cannot compare this error rate with other techniques. A one-way ANOVA analysis showed that the effect of density on finger error rate was not statistically significant $\left(F_{2,22}=0.291, \mathrm{~ns}\right)$.

To compare the finger error rates between the first and second experiments, we ran a t-test only for low and medium densities of the second study (which were the exact medium and high densities of the first study). We could not include the first study's low density or the second study's high density in the t-test since we did not have the same 
densities in the other experiment for them. The result of the t-test revealed that finger error rate in user study 2 was lower than user study $1\left(\mathrm{t}_{11}=1.79, p<0.05\right)$ for mentioned densities only, in line with our hypothesis (see Figure 41). This is likely because the improvements to the technique between experiments made it much easier for participants to tell how many fingers to use on the touchscreen. We suspect that most of the errors in user study 2 occurred when participants had to put three or four fingers on the screen. Since the phone used in this study was quite narrow, it is difficult to put all three or four fingers on the screen simultaneously. In the future, we plan to test the technique again using a larger touch screen like a tablet (more than 10 inch screen) to test this hypothesis.

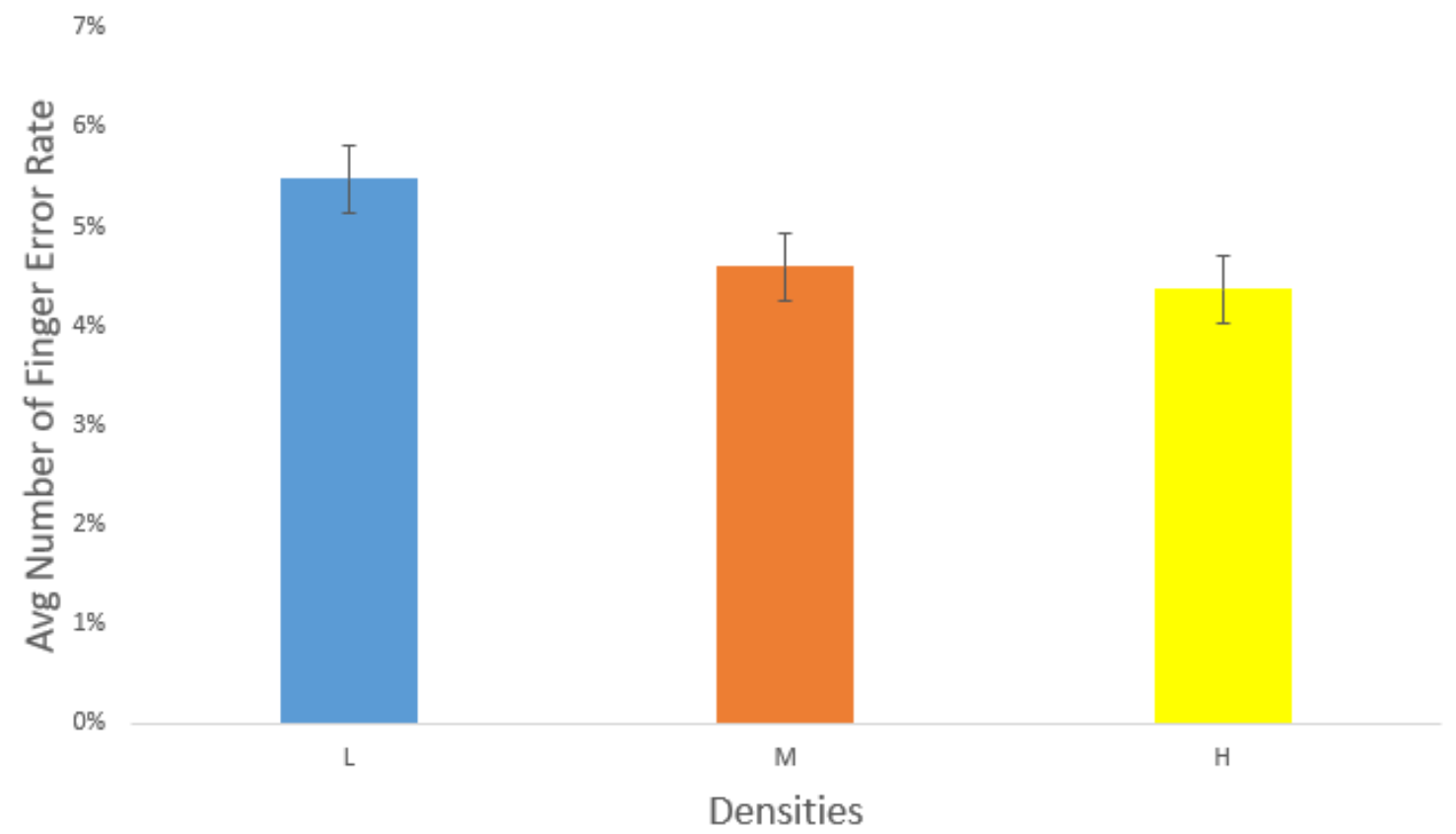

Figure 41: Result of one-way ANOVA analysis for finger error.

\subsubsection{Questionnaire Results}

We asked four usability questions from participants after finishing each technique and two questions after finishing the whole experiment to survey their experiences. The questions were about how easy, fast, and accurate each selection technique was and 
which their desired one was. We also asked them to explain the reasons for their selections, so we could better understand their requirements for further improvements. According to participants' responses, IMPReSS was most preferred (58\%), multi-touch was the second favorite (25\%), and progressive refinement was the least preferred (17\%). Participants mentioned that it was straightforward and faster to select the target object inside the menu in IMPReSS. However, some participants mentioned that the swiping action was more challenging when they had to put three or four fingers on the screen. Figure 42 shows the participants' responses to how easy, fast, and accurate each technique was based on the selection technique.

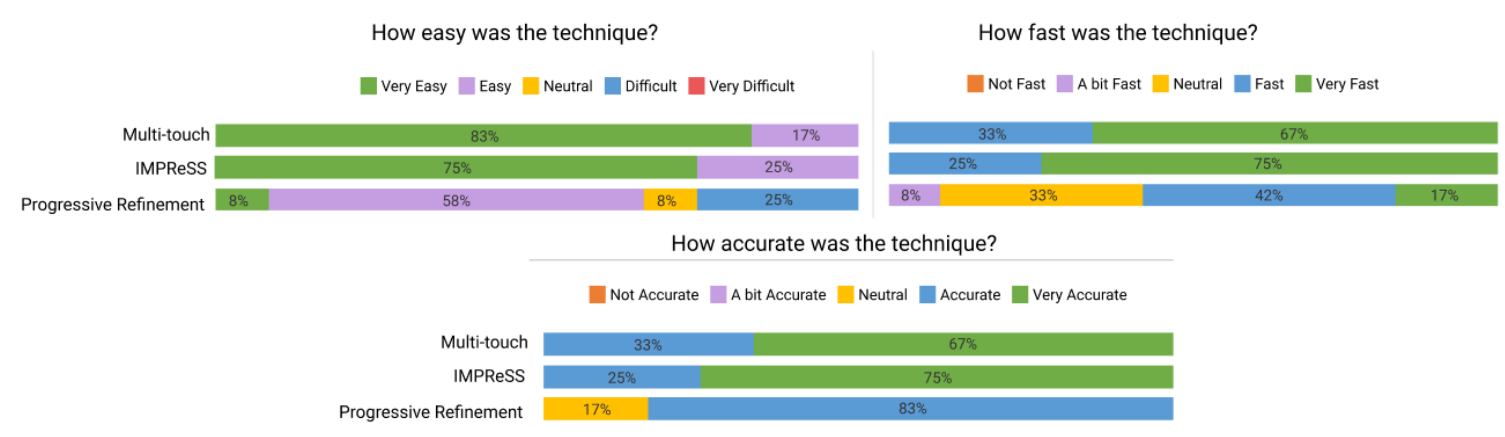

Figure 42: Selection technique usability and assessment questionnaire. Label numbers indicate the percentage of participants choosing each answer.

\subsection{Discussion}

Our experiment confirmed that search time is not dependent on the selection technique and is only influenced by density since finding the target book on shelves has the same mechanism in all techniques. The new "super" high density we included in this study increased search time, as seen in Figure 38, and supports that search time is highly dependent on density.

In contrast, selection time was impacted by both selection technique and density. According to our analysis, as seen in Figure 39, IMPReSS was faster than the other two 
techniques, which supported our hypothesis. This suggests that our improvement adding object position numbers inside quad menus - worked as intended, allowing participants to select the target object faster and requiring less time to think about how many fingers they had to put on the screen during selection.

Interestingly, there is little difference in selection time between low and medium density in IMPReSS. The reason is that most of the time, the finger error rate in low density is higher than in medium (see Figure 41). We believe that increasing the finger error rate has increased the selection time in low density since participants were learning the technique and tried to place the correct number of fingers on the screen. The multitouch technique was the second-fastest technique in this experiment. As seen in Figure 39, the IMPReSS and multi-touch techniques performed almost the same in selection time, except in high density (IMPReSS performed better). Like the first study, progressive refinement was slowest in this study, especially with high density.

Our analysis revealed that navigation error rate is dependent on selection technique but not density. Progressive refinement had the highest navigation error rate compared to the other techniques, especially with low and medium density. In progressive refinement, navigation errors occur when the user puts the selection ray in the incorrect quad menu or selects the line between the quad menu boundaries. Hence, with progressive refinement, high error rates can occur due to careless selection from users and selecting between boundaries. Another reason for that could be the imperfect calibration between the smartphone and the selection ray, which caused the high navigation error rate in progressive refinement. 
The finger error rate was lower in this study than in the first. This indicates that the new enhancement was helpful. Still, the low-density error rate is higher than in medium and high density, which may be related to unfamiliarity with the technique, as mentioned earlier.

\subsection{User Study Limitations}

Lack of pilot testing and tutorial/practice session for users, affected the experiment negatively, since we believe that users were learning about the technique during performance in the low density and got better in medium and high density.

In addition, ten out of 12 participants of the second study performed the first study as well, so they were familiar with the techniques, which might have affected their better performance in the IMPReSS technique.

Lack of density counterbalancing is another problem that may have some influence on the data and user experience as well.

Besides, performing user studies during the COVID-19 pandemic affected some critical parts of the experiment. Since participants had to set up all the equipment themselves, we had to consider some time for that and consequently limited the number of trials and densities to keep the length of the experiment reasonable (e.g., less than one hour). In addition, although we provided all the setup and experiment instructions in a PDF file and sent them online, and set some virtual meetings to explain some points, there were participants who needed help to perform the task.

\subsection{Conclusion}

Overall, this experiment revealed that IMPReSS is a better selection technique than the multi-touch and progressive refinement in terms of selection velocity, especially in a 
high-density environment. After that, multi-touch is the second-fastest technique, and finally, progressive refinement is the third. In terms of accuracy, IMPReSS is more precise than multi-touch. In contrast, progressive refinement is not accurate, especially in low and medium density. The result of the analysis shows that the enhancement we applied on the MTPR improved the selection time and accuracy in this technique. 


\section{Chapter 5: Conclusion}

We developed a selection technique in VR by combining progressive refinement with the CountMarks techniques with the intent of improving selection time and accuracy. We called the technique Multi-touch Progressive Refinement (MTPR). After the first study, we enhanced the technique and created the second variation, which we called IMPReSS.

This work focused on enhancing the two key factors of all selection tasks: selection time and accuracy (error rate). We ran two experiments to check these two factors in two variations of MTPR. Our selection time results were different in each experiment due to the improvements we made to our technique between the experiments. According to the result of selection time in the first experiment (see Figure 24), although fewer menus were opened when using MTPR, the multi-touch technique was faster, contrary to our hypothesis. We believe that the reason is that participants were unsure about the number of fingers required to select the desired object - many participants mentioned this in their questionnaires. This yielded higher selection times. Our second study was intended to determine whether resolving this issue would improve the technique. Thus, we ordered objects inside the menu and added labels to them to clearly indicate how many fingers would be required to select a given item. Familiarity with multi-touch from everyday smartphone and tablet use likely also played a role.

Results of the second study showed that our enhancement to the technique and improving distributing objects in menus decreased selection time, making IMPReSS the fastest technique in the second study compared to multi-touch and progressive refinement (see Figure 39). 
The navigation error rate in our technique was low for both MTPR and IMPReSS, which means that it was the most stable technique in terms of accuracy between the studies. See Figure 25 and Figure 40. In addition, the finger error rate for IMPReSS decreased considerably in the second study due to the technique enhancement (see Figure 41). Still, this error was high in the low-density environment in most cases, which could have been due to a lack of experience with the technique; as detailed earlier, participants always completed low density first. Therefore, we recommend some test trials (at least two) for all techniques before starting the experiment for participants to interact with techniques and get familiar with them. We also suggest counterbalancing density in future studies.

Finally, we found that search time is unaffected by the interaction technique but is strongly dependent on density. Of course, there could be other parameters, such as object size, which affect the search time, but they are beyond the scope of this thesis.

In the first user study, multi-touch was the most preferred technique since it was easy and fast. However, in the second study, the most preferred technique was IMPReSS. Most of the participants mentioned that the technique is clear and easy to use.

\subsection{IMPReSS Applications}

Due to the ongoing COVID-19 pandemic, many businesses are still inaccessible. IMPReSS could be helpful in virtual tours of places such as city libraries or museums. Users can easily select a desired book or object to read more about it in cluttered environments without expending much effort on selection. It could also be helpful in virtual learning scenarios, such as learning how to cook. When a user wants to pick 
objects (e.g., cooking utensils or ingredients) from shelves or cabinets, they can select their required object very quickly using this technique [42].

In addition, since both MTPR and IMPReSS are lowering the number of opened menus, it could be helpful in any situation that the number of selectable objects is high. For example, in any shooting game, we can use this technique to select or switch between guns in less time.

\subsection{Conducting Remote Experiments}

The biggest challenge we faced in this thesis was performing user studies during the COVID-19 pandemic since we had to carry out all user studies remotely. In this subsection, we summarize our experience running studies remotely during the COVID-19 pandemic.

The first problem was about designing the experiments. Since participants had to experiment in their homes, they needed extra time setting up the equipment.

Consequently, we had to reduce the number of trials and blocks to manage the whole experiment to be close to one hour. While we had considered including other conditions, like target size or additional densities, we decided to exclude these to keep the experiment length reasonable.

We also had to consider safety (e.g., participants bumping into things while in VR), hardware equipment disinfection protocols, and setup consistency between participants. To manage the safety problem, we asked participants to sit on a chair in front of the monitor and only move their hands close to themselves. Since participants did not need to stand, walk, or have large hand movements in the space, we were not worried about any bumping or injuries resulting from performing the experiment. To ensure all 
equipment was disinfected, we used a disposable face cover for HMD. We also sanitized all the hardware equipment after each use and/or quarantined them for 1-2 days; this process introduced bottlenecks into the experiment process.

Lastly, we installed a specific software on the smartphone. Consequently, we could not ask people who had personal VR hardware to experiment with their own equipment since they needed the prototype. Besides, each participant needed to have all the equipment in their place for at least a day to have adequate time to do the experiment. These problems caused more effort on our end and made the user study longer than we expected since we had to quarantine/sanitize equipment after each participant.

\subsection{Thesis Conclusion}

Two key factors we were aiming to achieve in this thesis were decreasing time in a selection technique while increasing the accuracy. Our experiments demonstrated that our new selection technique - which combined elements of CountMarks and progressive refinement - offered lower selection time and higher accuracy than the other two techniques. It was also shown in our experiments that object search time is not related to the technique at all, although unsurprisingly, density affects this.

We recommend using IMPReSS to select objects in highly dense VR environments. However, bigger target sizes were not included in this thesis and should be investigated in future studies. Also, we recommend using IMPReSS for applications when careful selection is not required in the first step.

Our experiments also revealed that the multi-touch technique is one of the users' favorite ones since it is easy to understand and interact with; nonetheless, IMPReSS is much faster and more accurate. 


\subsection{Future Work}

For future work, we would like to focus on increasing the number of menus from four to eight (based on the four cardinal and four intercardinal navigation directions), which means we need to add new swipe directions inside the menu as well. We would also like to investigate different object sizes in different densities to measure the influence of object size in our technique. It would also be noteworthy to test the technique with different touch screen sizes to see the effect of larger or smaller screens on the technique. Interacting with the technique with various user positions like standing or walking would also be an interesting idea to work. We can also mix this technique with voice commands. In that situation, rather than touching any screens, users can see their desired object's position in IMPReSS and select the object by voice command. Finally, we would like to design an experiment closer to real-world applications rather than focusing on selecting one specific object. 


\section{Appendices}

\section{Appendix A}

\section{A.1 COVID-19 Screening form}

\section{Screening Questions}

Project Title: Selection using multi-touch progressive refinement in VR

Clearance \#: 115567

Date of Clearance:

"This research has been cleared by Carleton University Research Ethics Board-B (CUREB-B Clearance \#115567)"

Did the person travel outside of Canada in the past 14 days?

Has the person tested positive for COVID-19 or had close contact with a confirmedcase of COVID-19 without wearing appropriate PPE?

Short answer text

Does the person have any of the following symptoms?• Fever • New onset of cough • Worsening * chronic cough • Shortness of breath• Difficulty breathing • Sore throat• Difficulty swallowing • Decrease of loss of sense of taste or smell• Chills Headaches• Unexplained fatigue/malaise/muscle aches (myalgias)• Nausea/vomiting, diarrhea, abdominal pain• Pink eye (conjunctivitis)• Runny nose or nasal congestion without other known cause

Short answer text 


\section{A.2 Invitation Email}

\section{Carleton \\ Canarda's Capital University}

This research has been cleared by Carleton University Research Ethics Board-B (CUREB-B Clearance \#115567)

Dear Participant,

Thank you so much for your interest in doing the VR experiment. The study's title is "Selection using multi-touch progressive refinement in VR " under the supervision of Dr. Rob Teather. This research has been cleared by Carleton University Research Ethics Board-B (CUREB-B Clearance \#115567). The study will take approximately 60 minutes, including setting up the equipment, and you will receive a \$15 Amazon gift card as compensation for your participation. To be eligible to do the study, no prior VR experience is required. You must be at least 18 years old and be able to read English. You must have normal or corrected-to-normal stereo 3D viewing capabilities - you must be able to see 3D effects in movies.

We would appreciate it if you pick a date from XXXX to XXXX in order to do the experiment. Due to the COVID-19 situation and as we mentioned in the poster, you will perform the experiment in your home. Hence we need your residence address as well as a selected date in response to this email. Please note that your address and contact information will be kept confidential, and they will not be stored permanently and will be destroyed upon returning the equipment.

After setting a suitable date, we will send you another email with related information about contactless drop-off and the "Setup and experiment instructions" file.

Please let us know if you have any questions or if there is any inconvenience.

Thank you so much in advance for your time,

Elaheh Samimi 


\title{
A.3 Contactless Drop-off Email
}

\author{
Garleton \\ Canada's Capital University
}

This research has been cleared by Carleton University Research Ethics Board-B (CUREB-B Clearance \#115567).

Dear Participants,

Thank you so much for choosing a date! Your selected date is XXXX, and your participant id is TBD.

You will receive a box including an Oculus Rift, two sensors, a computer, a smartphone (Samsung Galaxy S8) on YYYY (one day before experimenting). If you have any preferred time for drop-off, please let us know; otherwise, drop-off would be any time in the afternoon on the stated date. We will drop the box in front of your door contactlessly and by observing all COVID-19 protocols. We will stay near your residence (with following social distance protocols) to make sure you pick up the box. You will have all the equipment for one day, and we will pick them up on ZZZZ (one day after experimenting) at your preferred time and with the same process.

You can find the "Setup and experiment instructions" in the attached file.

Please don't hesitate to contact us if you have any questions or any concerns.

Thanks a lot,

Elaheh Samimi 


\section{A.4 Setup and Experiment Instructions}

CUREB-B Clearance \# 115567

\section{Carleton \\ U NIVERSITY \\ Canada's Capital University}

Step 1 - Consent Form

Please read carefully the consent form found here:

https://forms.gle/TU4S18SP3WfgnQk38

Step 2 - Pre-experiment Questionnaire

Please fill out the pre-experiment questionnaire found here:

https://forms.gle/CCsbPS1Jonpoxz5L6

Step 3 - Equipment Setup

Before we start the experiment, you will need to set up the base stations and the VR Head-Mounted Display (HMD).

1. What you will need:

a. A chair.

b. A table.

2. Place the sensors at 10 and 2 o'clock positions on the table $1-1.5 \mathrm{~m}$ apart.

3. Have them points toward the center of where you will sitting.

4. Sit at the center, 1-2m away from sensors.

5. Always face towards the sensors.

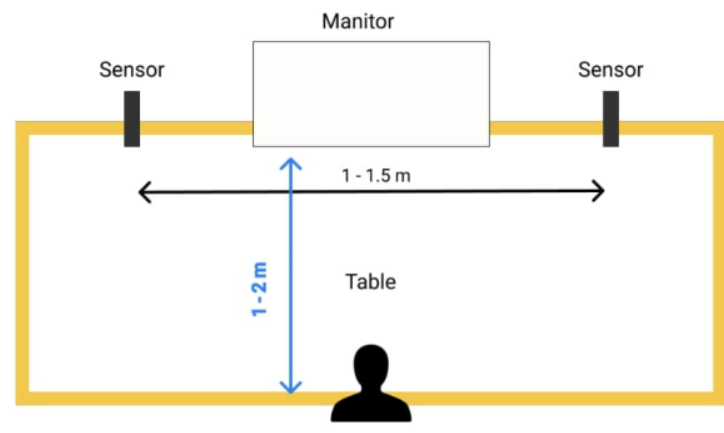

This research has been cleared by Carleton University Research Ethics Board-B (CUREB-B Clearance \#115567) 
CUREB-B Clearance \# 115567

*Note: For the best tracking experience, make sure both sensors can always see the headset.

You can watch the video in the below link to help you better understand the sensor positioning.

shorturl.at/iiqCO

Now you can move to the next part, which is connecting the equipment to the computer.

1. Connect the headset cable's HDMI connector to the HDMI port on the PC.

2. Connect the headset cable's USB connector to a USB 3.0 port on your compute

3. Connect the sensor cable to another USB 3.0 port. Once all three of these cables have been plugged in, you should see green icons next to them in the Oculus software, indicating they've been connected successfully.

Step 3.1 - Setting Up the Oculus Sensors

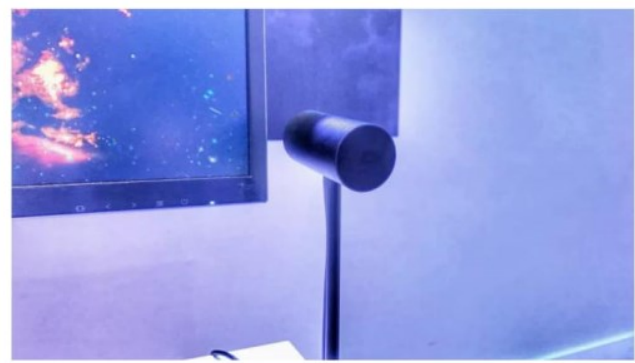

1. Open Oculus software, head to "Devices" in the menu, click "Configure Rift," and select "Sensor Setup."

2. Enter your height.

3. Place sensors between three and six feet from where your head will be during Rift use. Ensure nothing gets in the way between the sensor and where your headset would be, even if you crouch or move during use.

4. Hold the headset in front of you and move it around. You'll get a notification when the headset and sensor have connected.

5. Follow the steps again for the second sensor. 
Step 3.2 - Setting Up the Oculus Touch Controllers

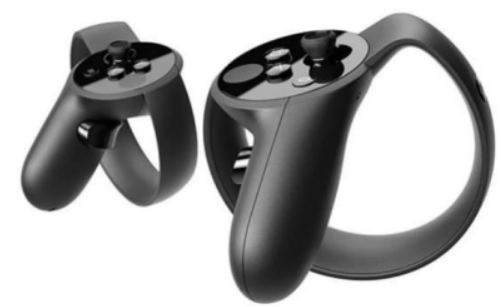

1. However, you don't need to use Rift controllers in this experiment; you have to set them up. After connecting the Oculus Sensors, you'll automatically be prompted to set up the Oculus Touch controllers. If you weren't prompted, head to the "Devices" menu, hit "Configure Rift," then press Add Left Touch.

2. Follow the on-screen instructions to pair the Left Touch controller.

3. Follow the process again for the Right Touch.

\section{Step 3.3 - Setting Up the Guardian System}

1. After setting up the Touch controllers, you'll automatically be prompted to set up the Guardian System, which maps out where you'll be using the Rift and warns you when you're near the boundaries of that area. If you weren't automatically prompted, head to "Configure Rift" and hit Guardian System in the Oculus software.

2. Follow the on-screen instructions to set up the Guardian System

In the end, if everything connected successful, Stream VR should look like this:

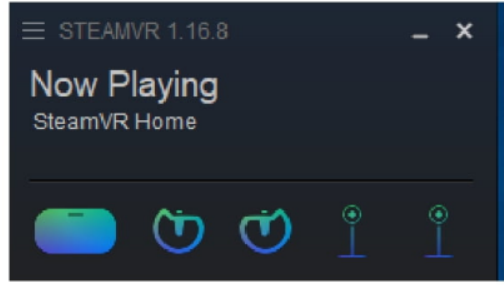

For more information, you can refer to the below link:

https://www.techradar.com/how-to/how-to-set-up-oculus-rift 
CUREB-B Clearance \# 115567

Step 4 - The Experiment

1. Next, you need to open the "Client-Server" application on the phone.

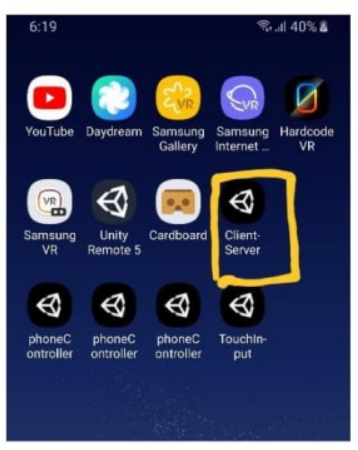

a. Please do not update the android version in case you get any message.

b. Please do not hit the "start server" button before running the application on the computer. Otherwise, the program will not work correctly.

2. Open the "CMPR_Experiment" folder on the desktop.

a. Once you open the folder, double click on CMPR_Experiment.exe to run the application.

3. Before you start the experiment, please make sure you have read the consent form and signed it.

4. In the setup menu window:

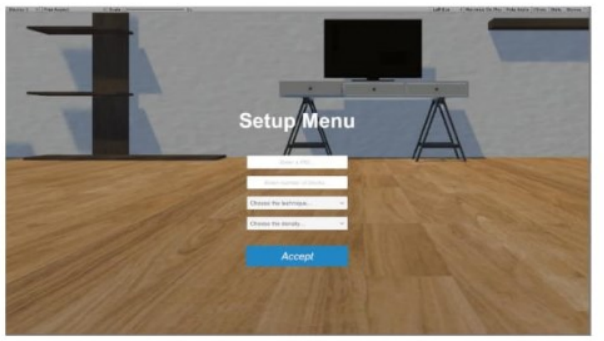

a. Enter your dedicated Participant ID. (you can find it in the email you have received from us)

b. Enter one (1) as the number of blocks.

This research has been cleared by Carleton University Research Ethics Board-B (CUREB-B Clearance \#115567) 
CUREB-B Clearance \# 115567
c. Choose "Progressive Refinement" as the technique.
d. Choose "Low" as density.
e. Hit the "Accept" button.
f. Now you can hit the "start server" button on the smartphone.

5. Wear the HMD

a. If you need help wearing that, please watch this video. https://www.youtube.com/watch?v=yGzfMFMYUX4

b. If you wear glasses, please watch this video. https://www.youtube.com/watch?v=LM1VIQOMRYI

6. You will see the rest instructions on how to perform the experiment in the VR scene.

7. After finishing the first round, you have to exit from the program. You can rest as long as you need here.

8. Whenever you feel ready, repeat steps 1 to 4 again, but this time change the density to "Medium" and do the experiment again.

9. For the third time, change the density to "High" and experiment again.

10. Please fill the form below when you finish the experiment with the progressive refinement method and all three densities. https://forms.gle/Qd99X6shXLMpmWca9

11. Repeat steps 1 to 9 for the "Count Mark" technique.

12. Please fill the form below when you finish the experiment with the count-mark method and all three densities. https://forms.gle/9jiidRTYDXAWErps5

13. Repeat steps 1 to 9 for the "Multitouch" technique.

14. Please fill the form below when you finish the experiment with the multitouch method and all three densities. https://forms.gle/yTJDMQySveQ3MVoa9

15. The whole experiment will be finished here.

\section{Step 5 - Post_experiment Questionnaire}

Please fill out the post-experiment questionnaire from here:

https://forms.gle/55RSgS6EPiDvnoGt8

This research has been cleared by Carleton University Research Ethics Board-B (CUREB-B Clearance \#115567) 


\section{A.5 Consent Form}

\section{Consent Form}

Project Title: Selection using multi-touch progressive refinement in VR

Clearance \#: 115567

Date of Clearance:

"This research has been cleared by Carleton University Research Ethics Board-B (CUREB-B Clearance \#115567)

${ }^{*}$ Required

Name and Contact Information of Researchers:

Elaheh Samimi

Email: ElahehSamimi@cmail.carleton.ca

Supervisor: Dr. Robert Teather

Email: Rob.Teather@carleton.ca

\section{Invitation}

You are invited to take part in a research experiment because you expressed your interest in participation. The information in this form is intended to help you understand what we are asking of you so that you can decide whether you agree to participate in this study.

To participate in this study, you must have normal or corrected to normal stereo vision (if you can see 3D effects in movies you have normal stereo vision) and be comfortable using a PC with an internet connection. You must not have COVID-19 or its symptoms such as dry cough and fever or be in selfisolation.

Your participation in this study is voluntary, and a decision not to participate will not be used against you in any way. As you read this form and decide whether to participate, please ask all the questions you might have, take whatever time you need, and consult with others as yo

What is the purpose of the study?

We are assessing the usability and performance of a new selection method in VR. The data will be a great help for designers and developers of VR systems, and the VR/ $\mathrm{HCl}$ research communities. 


\section{What will I be asked to do?}

If you agree to take part in the study, we will ask you to:

1. Setup the VR equipment in after equipment drop-off.

2. Answer a pre-experiment questionnaire.

3. Complete a series of selection tasks using a smartphone as the input device.

4. Answer usability and device assessment questionnaires after each method.

5. Answer post-experiment questionnaire..

6. Send the data logged by the software back to the researcher via email.

\section{Risks and Inconveniences}

The risks level involved with this experiment are minimal. Using a VR head-mounted display for a brief time ( 45 minutes), has an inherent small risk of cybersickness (e.g., nausea), but this is largely mitigated by the fact that you are seated stationary in this experiment (cybersickness typically presents when moving) and the current modern VR technology. Similarly, common VR-related risks (e.g., bumping into furniture) are also mitigated by you being seated.

To apply all COVID-19 protocols in all steps of our experiment, we check all participants' health about COVID-19 by asking some screening questions in another form before passing any equipment to them. If they are healthy and do the experiment, after that we carefully disinfected and/or quarantined all equipment before next participant use. Also, there is a disposable face mask in the package to use head mounted display. For the drop off process, we adhere to some kind of hand-off protocols, which means that we will put the equipment in front of your door, then distance sufficiently until you pick them up. We will apply the same process when we want to pick up the equipment from your place.

\section{Possible Benefits}

You may not receive any direct benefit from your participation in this study. However, your participation will assist future designers, researchers, and developers of VR in two ways. First, it may allow designers and researchers to better understand and measure user performance using a smartphone as an input device for a selection task in VR. Second, It will help researchers and developers measure the performance of this new selection method better and improve it in the future.

\section{Compensation/Incentives}

You will receive a $15 \$$ Amazon digital gift card. You will get a digital code sent to the same email address you used to initially contact the researcher within 2-3 days after the study is completed. This compensation will be paid to you even if you decide to withdraw from the study due to any reason. 


\section{No waiver of your rights}

By signing this form, you are not waiving any rights or releasing the researchers from any liability.

\section{Withdrawing from the study}

If you withdraw your consent during the course of the study, all information collected from you before your withdrawal will be discarded, unless you request that it be removed from the study data.

Once the experiment is complete you cannot withdraw, as your study data is provided anonymously and there is no way to link it back to your identity.

\section{Confidentiality}

The VR software will not be logging any identifying information in the study data. Other than on the consent forms, your name and information will not be stored. The online forms use a secure connection and will not store any personal information such as your IP or email address.

In case of equipment drop-off your address and contact information will be kept confidential and they will not be stored permanently and will be destroyed upon returning the equipment. Online video calls for questions and assistance with the setup and the experiment will be conducted via Zoom or skype and pre/post experiment assessments will be done via google forms. "In-session" data, such as the audio, video and chat transcript from the interview, will be stored locally on the researcher's computer. Operation data, such as meeting and performance data, will be stored and protected by Zoom, Skype and google on servers located in Toronto and Montreal, but may be disclosed via a court order or data breach. We will encrypt [or password protect] any research data that we store or transfer.

We will treat your personal information as confidential, although absolute privacy cannot be guaranteed. No information that discloses your identity will be released or published without your specific consent. Research records may be accessed by the Carleton University Research Ethics Board in order to ensure continuing ethics compliance.

The results of this study may be published or presented at an academic conference or meeting, but the data will be presented so that it will not be possible to identify any participants unless you give your express consent.

You will be assigned a participant ID so that your identity will not be directly associated with the study data you have provided. All data, including coded information, will be kept in a password-protected a secure computer. 


\section{Data Retention}

After the study is completed, your de-identified data will be retained for future research use for up to 3 years.

Contact information and address that we used for drop-off will be destroyed upon returning the VR hardware equipment.

\section{New information during the study}

In the event that any changes could affect your decision to continue participating in this study, you will be promptly informed.

\section{Ethics review}

This project was reviewed and cleared by the Carleton University Research Ethics Board B. If you have any ethical concerns with the study, please contact Carleton University Research Ethics Board (by email at ethics@carleton.ca.)

\section{Enter your name: *}

Your answer

\section{Statement of consent *}

I voluntarily agree to participate in this study. 


\section{A.6 Pre-Experiment Questionnaire}

\section{Pre-experiment Form}

This for is designed to be answered before doing the study.

* Required

Participant number *

Your answer

How old are you? *

Your answer

Do you have any VR experiences? *

Not at all

I used it a few times

I am a pro.

What is your dominant hand? *

$\bigcirc$ Left

Right 


\section{A.7 Usability and Device Assessment questionnaire _ MTPR and IMPReSS}

\section{Technique}

\section{Usability and device assessment - Count Mark}

This form is designed to be answered after doing Count-mark method.

* Required

Your participant ID *

Your answer

How was easy to select the book in this method? *

$\begin{array}{lllllll} & 1 & 2 & 3 & 4 & 5 & \\ \text { Very easy } & & & & & & \\ \text { Very difficult }\end{array}$

If it was difficult for you, what was the reason? (if it wasn't difficult please mention NA) *

Your answer 
How accurate was this selection method? *

$\begin{array}{lllllll} & 1 & 2 & 3 & 4 & 5 & \\ \text { Not accurate at all } & & \bigcirc & \bigcirc & \bigcirc & \bigcirc & \text { Very accurate }\end{array}$

How fast you could select the objects? *

Not fat at all $\quad \begin{array}{llllll}1 & 2 & 3 & 4 & 5 & \\ \text { Very fast }\end{array}$


A.8 Usability and Device Assessment questionnaire _ Multi-touch Technique

\section{Usability and device assessment - Multitouch}

This form is designed to be answered after doing Direct touch method.

* Required

Your participant ID *

Your answer

How was easy to select the book in this method? *

$\begin{array}{lllllll} & 1 & 2 & 3 & 4 & 5 & \\ \text { Very easy } & \bigcirc & \bigcirc & \bigcirc & \bigcirc & \bigcirc & \text { Very difficult }\end{array}$

If it was difficult for you, what was the reason? (if it wasn't difficult please mention NA) *

Your answer 
How accurate was this selection method? *

$\begin{array}{lllllll} & 1 & 2 & 3 & 4 & 5 & \\ \text { Not accurate at all } & \bigcirc & \bigcirc & \bigcirc & \bigcirc & \bigcirc & \text { Very accurate }\end{array}$

How fast you could select the objects? *

$\begin{array}{lllllll}1 & 2 & 3 & 4 & 5 & \\ \text { Not fat at all } & \bigcirc & \bigcirc & \bigcirc & \bigcirc & \bigcirc & \text { Very fast }\end{array}$




\section{A.9 Usability and Device Assessment questionnaire _ Progressive Refinement}

\section{Technique}

\section{Usability and device assessment - Progressive Refinement}

This form is designed to be answered after doing Progressive refinement method.

* Required

Your participant ID *

Your answer

How was easy to select the book in this method? *

$\begin{array}{lllllll} & 1 & 2 & 3 & 4 & 5 & \\ \text { Very easy } & \bigcirc & \bigcirc & \bigcirc & \bigcirc & \bigcirc & \text { Very difficult }\end{array}$

If it was difficult for you, what was the reason? (if it wasn't difficult please mention NA) *

Your answer 
How accurate was this selection method? *

$\begin{array}{lllllll}1 & 2 & 3 & 4 & 5 & \\ \text { Not accurate at all } & \bigcirc & \bigcirc & \bigcirc & \bigcirc & \bigcirc & \text { Very accurate }\end{array}$

How fast you could select the objects? *

1

2

3

4

5

Not fat at all $\bigcirc$

$\bigcirc$

$\bigcirc$

$\bigcirc$

Very fast 


\section{A.10 Post_Experiment Questionnaire}

\section{Post-experiment Form}

* Required

Your participant ID *

Your answer

Which Technique is your favourite selection method? (Please mentioned them from high priority to low priority.) 1.Progressive Refinement 2.Count_Mark 3. Multi-touch *

Your answer

Please explain the reason of your selection. 


\section{References}

[1] F. Argelaguet, C. Andujar, A survey of 3D object selection techniques for virtual environments, in: Comput. Graph., 2013: pp. 121-136.

[2] T. Babic, H. Reiterer, M. Haller, Pocket6: A 6DoF controller based on a simple smartphone application, in: SUI 2018 - Proc. Symp. Spat. User Interact., 2018: pp. $2-10$.

[3] F. Bacim de Araujo Silva, D.A. Bowman, R. Balakrishnan, Y. Cao, C. North, C.L. Nicholas F Polys, Increasing Selection Accuracy and Speed through Progressive Refinement, 2015.

[4] F. Bacim, R. Kopper, D.A. Bowman, Design and evaluation of 3D selection techniques based on progressive refinement, in: Int. J. Hum. Comput. Stud., Elsevier, 2013: pp. 785-802.

[5] G. Bailly, E. Lecolinet, Y. Guiard, Finger-count \& radial-stroke shortcuts: Two techniques for augmenting linear menus on multi-touch surfaces, in: CHI '10 Proc. SIGCHI Conf. Hum. Factors Comput. Syst., 2010: pp. 591-594.

[6] G. Bailly, E. Lecolinet, L. Nigay, Flower menus: a new type of marking menu with large menu breadth, within groups and efficient expert mode memorization, in: AVI '08 Proc. Work. Conf. Adv. Vis. Interfaces, 2008: pp. 15-22.

[7] G. Bailly, É. Lecolinet, L. Nigay, Visual Menu Techniques, in: ACM Comput. Surv. Assoc. Comput. Mach., 2017.

[8] R.A. Bolt, "Put-that-there": Voice and gesture at the graphics interface, in: SIGGRAPH '80 Proc. 7th Annu. Conf. Comput. Graph. Interact. Tech., 1980: pp. $262-270$. 
[9] C.W. Borst, A.P. Indugula, Realistic virtual grasping, in: IEEE Proceedings. VR 2005. Virtual Real., 2005: pp. 91-99.

[10] D.A. Bowman, L.F. Hodges, Evaluation of techniques for grabbing and manipulating remote objects in immersive virtual environments, in: I3D '97 Proc. 1997 Symp. Interact. 3D Graph., 1997: pp. 35-38.

[11] D.A. Bowman, R.P. McMahan, Virtual reality: How much immersion is enough?, in: Computer (Long. Beach. Calif)., 2007: pp. 36-43.

[12] J. Cashion, C. Wingrave, J.J. Laviola, Dense and dynamic 3D selection for gamebased virtual environments, in: IEEE Trans. Vis. Comput. Graph., 2012: pp. 634642.

[13] H.-H. Chen, Y.-B. Lin, R.-H. Liou, Direction-based Wireless Remote Controller: A Smartphone Application, in: J. Wirel. Mob. Networks Ubiquitous Comput. Dependable Appl., 2011: pp. 33-45.

[14] H.G. Debarba, J.G. Grandi, A. Maciel, L. Nedel, R. Boulic, Disambiguation canvas: A precise selection technique for virtual environments, in: Kotzé P., Marsden G., Lindgaard G., Wesson J., Winckler M. Human-Computer Interact. INTERACT 2013, 2013: pp. 388-405.

[15] A.A. Didehkhorshid, Performance Evaluation of Warped Virtual Surfaces in Virtual Reality by Affairs in partial fulfillment of the requirements for the degree of in, in: 2020.

[16] Y. Farmani, R.J. Teather, Evaluating discrete viewpoint control to reduce cybersickness in virtual reality, in: Virtual Real., Springer London, 2020: pp. 645664. 
[17] J.D. Fekete, N. Elmqvist, Y. Guiard, Motion-pointing: Target selection using elliptical motions, in: Conf. Hum. Factors Comput. Syst. - Proc., 2009: pp. 289298.

[18] S. Frees, G.D. Kessler, E. Kay, PRISM interaction for enhancing control in immersive virtual environments, in: ACM Trans. Comput. Interact., 2007.

[19] E.L. Gilles Baillya ,Jörg Müllera, Design and evaluation of finger-count interaction: Combining multitouch gestures and menus, Int. J. Hum. Comput. Stud. (2012) 673-689.

[20] T. Grossman, The bubble cursor: enhancing target acquisition by dynamic resizing of the cursor's activation area, in: SIGCHI Conf. Hum., 2005: pp. 281-290.

[21] T. Grossman, R. Balakrishnan, The design and evaluation of selection techniques for 3D volumetric displays, in: UIST '06 Proc. 19th Annu. ACM Symp. User Interface Softw. Technol., 2006: pp. 3-12.

[22] G. Guidi, L.L. Micoli, C. Casagrande, L. Ghezzi, Virtual reality for retail, in: 2010 16th Int. Conf. Virtual Syst. Multimedia, VSMM 2010, 2010: pp. 285-288.

[23] D.A. Guttentag, Virtual reality: Applications and implications for tourism, in: Tour. Manag., 2010: pp. 637-651.

[24] G. De Haan, M. Koutek, F.H. Post, IntenSelect: Using dynamic object rating for assisting 3D object selection, in: EGVE'05 Proc. 11th Eurographics Conf. Virtual Environ., 2005: pp. 201-209.

[25] M. Hofer, T. Hartmann, A. Eden, R. Ratan, L. Hahn, The Role of Plausibility in the Experience of Spatial Presence in Virtual Environments, in: Front. Virtual Real., 2020: pp. 1-9. 
[26] J. Jacobs, B. Froehlich, A soft hand model for physically-based manipulation of virtual objects, in: 2011 IEEE Virtual Real. Conf., IEEE, 2011: pp. 11-18.

[27] N. Katzakis, R.J. Teather, K. Kiyokawa, H. Takemura, INSPECT: extending plane-casting for 6-DOF control, in: Human-Centric Comput. Inf. Sci., Springer Berlin Heidelberg, 2015.

[28] J.I. Kiger, The depth/breadth trade-off in the design of menu-driven user interfaces, in: Int. J. Man. Mach. Stud., 1984: pp. 201-213.

[29] S. Knoedel, M. Hachet, Multi-touch RST in 2D and 3D spaces: Studying the impact of directness on user performance, in: 2011 IEEE Symp. 3D User Interfaces, 2011: pp. 75-78.

[30] R. Kopper, F. Bacim, D.A. Bowman, Rapid and accurate 3D selection by progressive refinement, in: 3DUI 2011 - IEEE Symp. 3D User Interfaces 2011, Proc., 2011: pp. 67-74.

[31] R. Kopper, D.A. Bowman, M.G. Silva, R.P. McMahan, A human motor behavior model for distal pointing tasks, in: Int. J. Hum. Comput. Stud., 2010: pp. 603-615.

[32] A. Kulshreshth, J.J. La Viola Jr, Exploring the usefulness of finger-based 3D gesture menu selection, in: CHI '14 Proc. SIGCHI Conf. Hum. Factors Comput. Syst., 2014: pp. 1093-1102.

[33] W. Kun, H. Zong, Application Study of Virtual Reality in Real Estate Industry, in: Proc. 3rd WSEAS Int. Conf. Comput. Eng. Appl., 2009: pp. 247-251.

[34] G. Kurtenbach, W. Buxton, The limits of expert performance using hierarchic marking menus, in: Proc. INTERACT'93 CHI'93, 1993: pp. 482-487.

[35] G. Kurtenbach, W. Buxton, User Learning and Performance with Marking Menus, 
in: CHI '12 Proc. SIGCHI Conf. Hum. Factors Comput. Syst., 2012: pp. 22212230.

[36] G.P. Kurtenbach, The design and evaluation of superconducting connectors, 1993.

[37] J.J. Laviola, E. Kruijff, R.P. McMahan, D.A. Bowman, I. Poupyrev, 3D User Interfaces: Theory and Practice, 2017.

[38] G.J. Lepinski, T. Grossman, G. Fitzmaurice, The design and evaluation of multitouch marking menus, in: CHI '10 Proc. SIGCHI Conf. Hum. Factors Comput. Syst., 2010: pp. 2233-2242.

[39] J. Liang, G. Mark, JDCAD: A highly interactive 3D modeling system., in: Comput. Graph., 1994: pp. 499-506.

[40] D. Mendes, D. Medeiros, M. Sousa, E. Cordeiro, A. Ferreira, J.A. Jorge, Design and evaluation of a novel out-of-reach selection technique for VR using iterative refinement, in: Comput. Graph., Elsevier Ltd, 2017: pp. 95-102.

[41] M.R. Mine, Virtual environment interaction techniques, in: Virtual Environ. Interact. Tech., 1995: pp. 1-18.

[42] Ma.R. Mine, F.P. Brooks Jr, C.H. Sequin, Moving Objects In Space: Exploiting Proprioception In Virtual-Environment Interaction, in: SIGGRAPH '97 Proc. 24th Annu. Conf. Comput. Graph. Interact. Tech., 2017: pp. 153-169.

[43] Oculus Blog, Quest 2, The next generation of all-in-onr VR, (2020).

[44] V.S. Pantelidis, D.C. Vinciguerra, Virtual reality in education, in: Teach. Learn. with Technol. Beyond Constr., 2010: pp. 151-181.

[45] F. Periverzov, H. Ilieş, IDS: The intent driven selection method for natural user interfaces, in: 2015 IEEE Symp. 3D User Interfaces, 3DUI 2015 - Proc., 2015: pp. 
$121-128$.

[46] Photon Blog, Introduction | Photon Engine, (2021).

[47] J.S. Pierce, A. Forsberg, M.J. Conway, S. Hong, R. Zeleznik, M.R. Mine, Image plane interaction techniques in 3D immersive environments, in: I3D '97 Proc. 1997 Symp. Interact. 3D Graph., 1997: pp. 39-43.

[48] J. Pollock, R.J. Teather, CountMarks: Multi-Finger Marking Menus for Mobile Interaction with Head-Mounted Displays, in: Int. Conf. Human-Computer Interact., 2019: pp. 1-20.

[49] I. Poupyrev, M. Billinghurst, S. Weghorst, T. Ichikawa, Go-Go interaction technique: non-linear mapping for direct manipulation in VR, in: UIST '96 Proc. 9th Annu. ACM Symp. User Interface Softw. Technol., 1996: pp. 79-80.

[50] I. Poupyrev, S. Weghorst, M. Billinghurst, T. Ichikawa, Egocentric Object Manipulation in Virtual Environments: Empirical Evaluation of Interaction Techniques, in: Comput. Graph. Forum, 1998: pp. 41-52.

[51] M. Prachyabrued, C.W. Borst, Design and Evaluation of Visual Interpenetration Cues in Virtual Grasping, in: IEEE Trans. Vis. Comput. Graph., 2016: pp. 17181731.

[52] G. Ren, E. O'Neill, 3D Marking menu selection with freehand gestures, in: 2012 IEEE Symp. 3D User Interfaces, IEEE, 2012: pp. 61-68.

[53] A. Rizzo, T.D. Parsons, B. Lange, P. Kenny, J.G. Buckwalter, B. Rothbaum, J.A. Difede, J. Frazier, B. Newman, J. Williams, G. Reger, Virtual reality goes to war: A brief review of the future of military behavioral healthcare, in: J. Clin. Psychol. Med. Settings, 2011: pp. 176-187. 
[54] G. Salvendy, Manual control and tracking. Handbook of human factors, New York: Wiley, 1987.

[55] B. Shneiderman, Direct Manipulation: A Step Beyond Programming Languages, in: CHI '81 Proc. Jt. Conf. Easier More Product. Use Comput. Syst. (Part - II) Hum. Interface User Interface - Vol. 1981, 1983: pp. 57-69.

[56] A.L. Simeone, Indirect touch manipulation for interaction with stereoscopic displays, in: 2016 IEEE Symp. 3D User Interfaces, 2016: pp. 13-22.

[57] A. Steed, C. Parker, 3D Selection Strategies for Head Tracked and Non-Head Tracked Operation of Spatially Immersive Displays, in: 8th Int. Immersive Proj. Technol. Work., 2004: pp. 13-14.

[58] S. Strothoff, D. Valkov, K. Hinrichs, Triangle cursor: Interactions with objects above the tabletop, in: ITS '11 Proc. ACM Int. Conf. Interact. Tabletops Surfaces, 2011: pp. 111-119.

[59] E.E. Swartz, R.T. Floyd, M. Cendoma, Cervical Spine Functional Anatomy and the Biomechanics of Injury Due to Compressive Loading, J. Athl. Train. 40 (2005) 155.

[60] A. Talvas, M. Marchal, A. Lecuyer, The god-finger method for improving 3D interaction with virtual objects through simulation of contact area, in: IEEE Symp. 3D User Interface 2013, 3DUI 2013 - Proc., IEEE, 2013: pp. 111-114.

[61] R.J. Teather, W. Stuerzlinger, Pointing at 3D targets in a stereo head-tracked virtual environment, in: 3DUI 2011 - IEEE Symp. 3D User Interfaces 2011, Proc., 2011: pp. 87-94.

[62] R.J. Teather, W. Stuerzlinger, Pointing at 3D target projections with one-eyed and 
stereo cursors, in: Conf. Hum. Factors Comput. Syst. - Proc., 2013: pp. 159-168.

[63] S. Thompson, VR Applications: 21 Industries already using Virtual Reality, (2020).

[64] Unity team, Wondering what Unity is? Find out who we are, where we've been and where we're going | Unity, (n.d.).

[65] Unreal engine, The most powerful real-time 3D creation platform - Unreal Engine, (2021).

[66] L. Vanacken, T. Grossman, K. Coninx, Exploring the effects of environment density and target visibility on object selection in 3D virtual environments, in: 2007 IEEE Symp. 3D User Interfaces, 2007: pp. 115-122.

[67] J. Velasco, VR controllers the good the bad and the ugly - YouTube, (2016).

[68] Virtual Reality Society, History Of Virtual Reality - Virtual Reality Society, (2019).

[69] S. Zhai, I. Poupyrev, M. Billinghurst, S. Weghorst, T. Ichikawa, Human performance in six degree of freedom input control, in: UIST (User Interface Softw. Technol. Proc. ACM Symp., 1996: pp. 79-80.

[70] S. Zhao, M. Agrawala, K. Hinckley, Zone and Polygon menus: Using relative position to increase the breadth of multi-stroke marking menus, in: CHI '06 Proc. SIGCHI Conf. Hum. Factors Comput. Syst., 2006: pp. 1077-1086.

[71] C.B. Zilles, J.K. Salisbury, Constraint-based god-object method for haptic display, in: Proc. 1995 IEEE/RSJ Int. Conf. Intell. Robot. Syst. Hum. Robot Interact. Coop. Robot., 1995: pp. 146-151. 